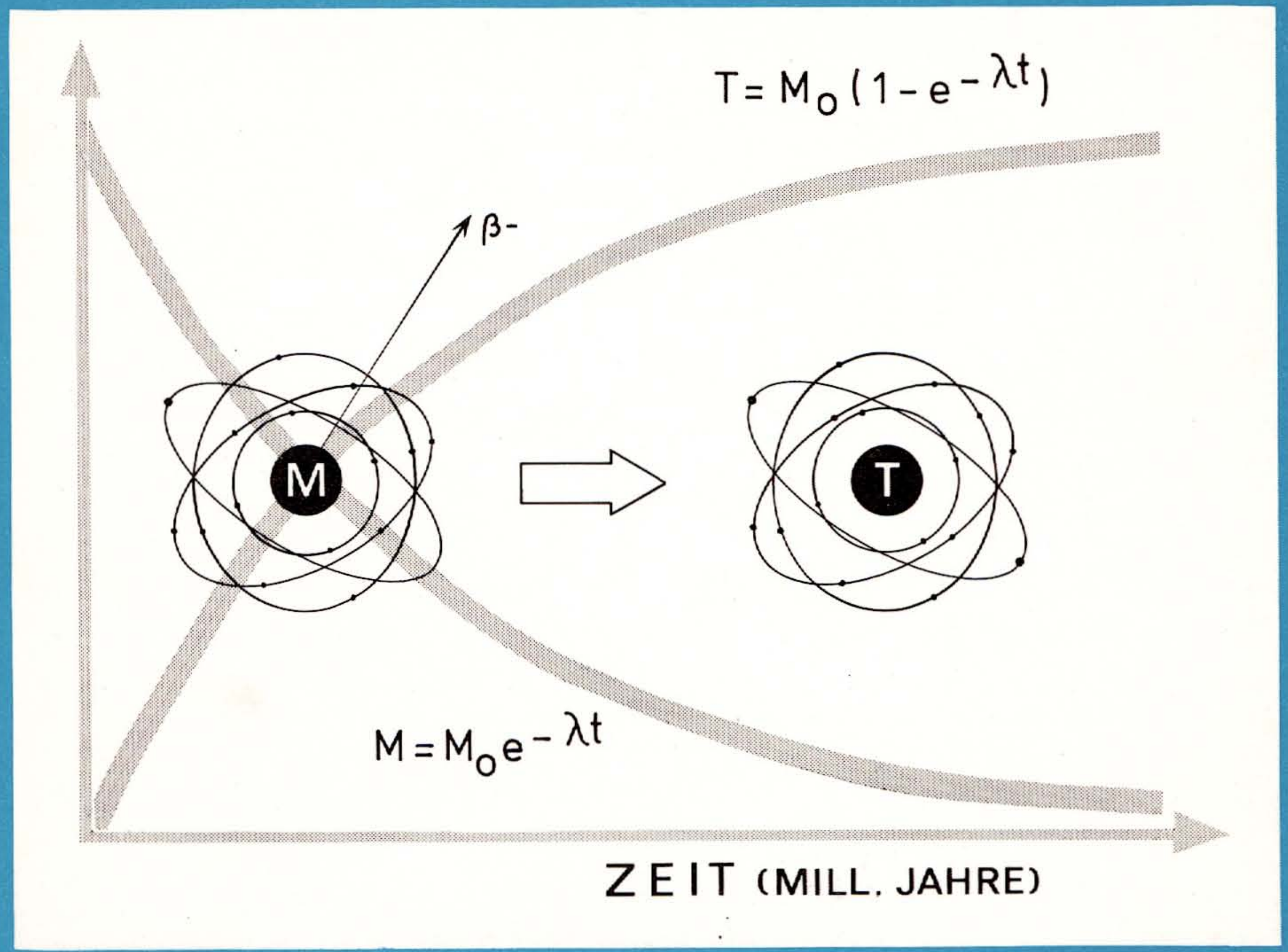

\title{
RADIOMETRISCHE METHODEN in der GEOCHRONOLOGIE
}

\author{
von I. WENDT
}

mit Beiträgen von:

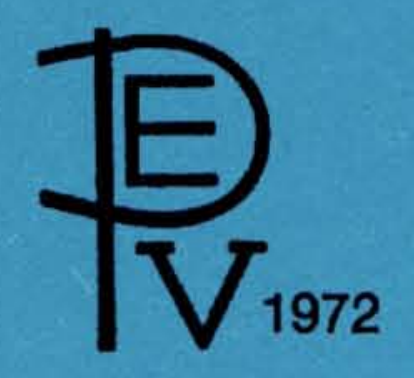

H. LENZ und M. SCHOELL 


\title{
CLAUSTHALER TEKTONISCHE HEFTE
}

\author{
Herausgeber: \\ o. Professor Dr. Andreas Pilger
}

Redaktion:

Universitätsdozent Dr. Horst Quade

\author{
Anschrift: \\ Geologisches Institut \\ der Technischen Universität Clausthal \\ D-3392 CLAUSTHAL-ZELLERFELD \\ Leibnizstraße
}




\title{
RADIOMETRISCHE METHODEN in der GEOCHRONOLOGIE von I. WENDT
}

\author{
mit Beiträgen von: \\ H. LENZ und M. SCHOELL \\ unter redaktioneller Mitarbeit von: \\ H. FLICK
}

\section{Seiten. 47 Abbildungen im Text, 5 Tabellen und 1 Abbildung im Anhang}

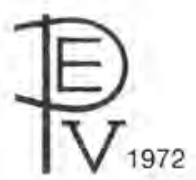


Alle Rechte vorbehalten, insbesondere das der Obersetzung;

photomechanische Wiedergabe, auch auszugsweise nur mit schriftlicher Genehmigung des Verlages Copyright by Verlag Ellen Pilger, Clausthal-Zellerfeld 1971 - Printed in Germany

Druck: Paul Krösing KG, Osterode am Harz 
Mit der stetig zunehmenden Anwendung von Isotopenuntersuchungen für geologische Fragestellungen hat auch die radiometrische Altersbestimmung im letzten Jahrzehnt sehr an Bedeutung gewonnen.

Das vorliegende Buch soll dem Geowissenschaftler elnen Uberblick über die gängigen Methoden der radiometrischen Altersbestimung, deren Grundlagen und Anwendungsbereiche geben. Es wird daher in den ersten Kapiteln die theoretische Basis dieser Verfahren in leicht verständicher Form aufgezeichnet, wobel bewußt auf kompliziertere mathematische Ableitungen verzichtet wurde zugunsten von anschaulichen graphischen Darstellungen.

Anhand von praktischen Beispielen wird die Anwendung der verschiedenen Methoden und die Interpretation der Ergebnisse diskutiert.

Das Heft ist sowohl als Textbuch für eine Vorlesung als auch zum Selbststudium für Geologen, Mineralogen und Geophysiker gedacht. 
1. Einführung und historischer Uberblick 1

2. Die Isotopenverdünungsmethode $\quad 6$

3. Die $\mathrm{Rb} / \mathrm{Sr}$-Methode 14

3.1 Einleitung 14

3.2 Die Datierung von Mineralen 15

3.3 Die Isochronenmethode 19

3.4 Das Compston-Jeffery-Verfahren 24

4. Die K/Ar-Methode 30

4.1 Isotope des Kaliums, Konstanten und Formeln 30

4.2 Störungen des K/Ar-Verhältnisses 33

4.3 Korrektur der Alterswerte bei Diffusionsverlust 40

4.4 Die Kaliumanalyse 42

4.5 Die Bestimmung des radiogenen Argons 44

5. Die Uran/Blei-Methode 49

5.1 Die direkten Uran-Ble1-Methoden 49

5.2 Die abgeleitete Blei-Blei-Methode 52

5.3 Diskordante Uran-Blei-Alter 56

5.4 Die ${ }^{210} \mathrm{~Pb}$-Methode 66

5.5 Die Fission-track-Methode 69

6. Die Blei-Blei-Methode 75

6.1 Die Isotopenverhältnisse des gewöhnlichen Bleies 75

6.2 Das Holmes-Houtermans-Modell 76

6.3 Störungen des Holmes-Houtermans-Modells 80

6. 4 Das Russe1-Farquhar-Cumming-Mode11 82

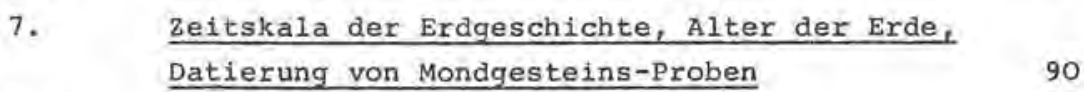

7.1 Die Zeitskala der Erdgeschichte 90

7.2 Das Alter der Erde 92

7.3 Datierung von Mondgestein 97 
8. Radiometrische Altersbestimmung am

Brockenintrusivkomplex im Harz als Bei-

spiel der Interpretation diskordanter

Modellalter

102

8.1 Einleitung 102

8.2 Kurze geologische Ubersicht 104

8.3 K/Ar-Datierung an Biotit-Chlorit-Gemischen 106

8.3.1 Das Datierungsmaterial 106

8.3.2 Der Einfluß des Chloritgehaltes auf das $\mathrm{K} / \mathrm{Ar}-\mathrm{Modellalter}$

8.3.3 Korrektur der Modellalter 112

8.4 Rb/Sr-Datierung 115

8.4.1 Gesamtgesteinsanalysen am Brocken- und
Oker-Granit 115

8.4.2 Gesamtgesteinsanalysen an elnem Aplitgranitgang als Beispiel für Sr-Homogenisierung im Gesteinsbereich

8.4.2.1 Kurze petrographische Beschreibung

8.4.2.2 Allgemeines zur Sr-Homogenisierung im

8.4.2.3 Die Ergebnisse und deren Diskussion

8.5 AbschluB der geologischen Interpretation 


\section{EINFUHRUNG UND HISTORISCHER UBERBLICK}

Auf die Möglichkeit, mit Hilfe von langlebigen, natürlichen, radioaktiven Isotopen geologische Zeiten messen zu können, wurde schon lange bevor genaue massenspektrometrische MeBmethoden zur Bestimmung von Isotopenhäufigkeiten existiexten, hingewiesen. Und es ist natürlich, daß man zunächst nur an das am längsten bekannte natürliche radioaktive Element, an das Uran gedacht hat. So konzentrierten sich die Bemühungen, die durch die Entdeckung der natürlichen Radioaktivität neu eröffnete Methode der Messung geologischer Zeiten anzuwenden, zunächst auf Uranminerale, Es gibt hier zwei grundsätzlich verschiedene Wege: die im folgenden eingehend beschriebene Uran-Blei-Methode, wobei aus der Menge des radiogenen stabilen Endproduktes, dem ${ }^{206} \mathrm{~Pb}$ und ${ }^{207} \mathrm{~Pb}$, im Verhältnis zum radioaktiven Mutterelement Uran das Alter berechnet wird, und die Uran-, Thorium-Helium-Methode, die auf der Tatsache beruht, daB bei einem Zerfall eines Uranatoms auf dem Wege des weiteren Zerfalls bis zu seinem stabilen Endprodukt Blei acht $\alpha$-zerfälle (beim ${ }^{235} \mathrm{U} \rightarrow{ }^{207} \mathrm{~Pb}$ sieben $\alpha$-Zerfälle) und beim Zerfall des Thoriums ${ }^{232}$ Th sechs $\alpha$-Zerfälle stattfinden. Da die $\alpha$-Teilchen aber Helium-Kerne sind, ist die Menge des in einem uran- und thoriumhaltigen Mineral gefundenen Heliums ein MaB für die zeitdauer, in der dieser zerfall stattgefunden hat, sofern das entstandene Helium wăhrend der ganzen geologischen Geschichte des Minerals nicht teilweise aus diesem entwichen ist. Es handelt sich hier um die gleiche Voraussetzung wie bei der heute so erfolgreich angewendeten K/ArMethode, wo das radiogene Endprodukt ebenfalls ein Edelgas ist. In den meisten Fällen erfüllen die Uranminerale die Voraussetzung, das entstandene Helium vollständig behalten zu haben, nicht so gut wie geeignete Kaliuminerale bezüglich des Argons.

Das lelcht diffundierende Helium ist meist zu einem großen Teil aus den Uranmineralen entwichen, und die mit dieser Methode erhaltenen "Alter" sind im allgemeinen erheblich zu 
klein. Bei der Anwendung der Uran-Blei-Methode, auf die bereits HOLMES im Jahre 1913 hingewiesen hat, bestand zunächst die Schwierigkeit, zwischen primär im Uranmineral zur zeit seiner Bildung bereits enthaltenen Blei und dem durch radioaktiven Zerfall entstandenen unterscheiden zu können. Die ersten durch chemische Blei- und Uranbestimmung erhaltenen $\mathrm{U} / \mathrm{Pb}-$ Alter waren daher auch im allgemeinen größer als das tatsächliche Bildungsalter. Diese Methode wurde durch eine exakte Atorngewichtsbestimmung des Bleies, mit deren Hilfe die Anteile von Zerfallsblei und gewöhnlichem Blel annähernd bestimmt werden konnten, weiterhin verbessert, aber erst durch die Einfuhrung der massenspektrometrischen Verfahren waren zuverlässige Bestimmungen der Isotope des Zerfallsbleies möglich geworden. Die Verbesserung der Analysenmethoden erlaubten sehr genaue Blei- und Urankonzentrationsbestimmungen, auch bei kleineren Uran- bzw. Thoriumkonzentrationen, so daB man nicht mehr in der Anwendung dieser Datierungsmethode auf Uranminerale, die in der Natur nicht sehr häufig sind, beschränkt war, sondern auch akzessorische Minerale, wie zirkon, Titanit u.ä. datieren kann, womit der Anwendungsbereich der U/Pb-Methode auf die meisten magmatischen Gesteine ausgedehnt werden konnte.

Die Existenz eines langlebigen Rb-Isotops ist schon seit dem Jahre 1905 bekannt, aber erst 1937 wurde von HAHN, STRASSMANN un WALLING und MATTAUCH das ${ }^{87} \mathrm{Rb}$ als das radioaktive Isotop identifiziert. Da Rubidium ein realtiv weit verbreitetes Element ist, wurde die Möglichkeit, dem ${ }^{87} \mathrm{Rb} \longrightarrow^{87} \mathrm{Sr}$ Zerfall für eine geologische zeitmessung an geeigneten Mineralen zu benutzen, klar erkannt. Wegen der Schwierigkeit mit den damaligen technischen Möglichkeiten, die wegen der großen Halbwertszeit des ${ }^{87} \mathrm{Rb}$ nur sehr kleinen Mengen ${ }^{87} \mathrm{Sr}$ genügend genau zu bestimmen, konnten die ersten $\mathrm{Rb} / \mathrm{Sr}$-Datierungen auch nur an sehr $\mathrm{Rb}-\mathrm{rei}-$ chen Mineralen, die im Vergleich zur Menge des Zerfalls-Sr praktisch kein gewöhnliches $\mathrm{Sr}$ enthielten, wie z.B. Lepidolithen durchgeführt werden. Erst mit Hilfe der massenspektrometrischen Isotopenverdinnungsmethode war es möglich, auch Minerale, die weniger Rubidium enthalten, wie z.B. Biotit und Muskowit, zu datieren. Damit war nach dieser Methode ein weites Anwendungs- 
feld eröffnet worden. Das später entwickelte $\mathrm{Rb} / \mathrm{Sr}$-Isochronenverfahren, angewendet auf Gesamtgesteinsproben einerseits und abgetrennte Minerale andererseits, erlaubt eine weitergehende Interpretation, auch bei einer komplizierten geologischen Geschichte der untersuchten Gesteinseinheit, so daß es möglich ist, nicht nur das primäre Gesteinsbildungsalter, sondern auch das Alter eines späteren metamorphen Einflusses zu bestimmen. Damit war eine weitere Datierungsmethode mit einem breiten Anwendungsgebiet eingefürt.

Ebenfalls im Jahre 1905, als die Radioaktivität des Rubidiums entdeckt wurde, erkannte man, daB auch das Element Kalium B-Strahlung aussendet, und im Jahre 1928 wurde von KOHLHÖRSTER die $Y$-Aktivität des Kaliums bei Aktivitätsmessungen in Kalibergwerken entdeckt. Nachdem aufgrund theoretischer Betrachtungen NEWMAN und WALKE 1935 ein bis dahin noch unbekanntes Isotop des Kaliums, das ${ }^{40} \mathrm{~K}$ als ursache für die beobachtete Radioaktivität postuliert hatten, konnte NIER im selben Jahr dieses Isotop auch nachweisen und seine Häufigkeit von $1,19 \times 10^{-4}$ ermitteln. C.F. v. WEIZSÄCKER SchloB aus der annormal hohen Häufigkeit des ${ }^{40} \mathrm{Ar}$ im atmosphärischen Argon, daB dieses Argon zum größten Teil aus dem $Z$ erfall des ${ }^{40} O_{K}$ entstanden sein müste, und zwar durch einen Zerfall, den man nur aufgrund theoretischer Uberlegungen von Chr. MøLLER (1937) kannte, dem $\mathrm{K}$-Einfang. Es gelang dann im Laufe der folgenden Jahre, das Zerfallsschema des $40_{K}$ aufzustellen und die Zerfallskonstanten des $\beta$-Zerfalls und des K-Einfangs zu ermitteln. 1948 haben NIER und ALDRICH aus Kaliummineralen Argon extrahiert und ein erhöhtes ${ }^{40} \mathrm{Ar} /{ }^{36} \mathrm{Ar}$-Verhältnis im Vergleich $\mathrm{zu}$ atmosphärischem Argon gefunden, womit der erste Schritt in Richtung einer K/Ar-Altersbestimung getan war. GENTNER und Mitarbeiter haben dann in den 1950-iger Jahren die ersten K/Ar-Datierungen durchgefuhrt. Man fand bald, daB Minerale, wie Muskowit, Biotit und andere Glimmer für die K/Ar-Datierung besonders gut geelgnet sind und daB, sofern das Gestein keinen weiteren Erwärmungen in Laufe seiner geologischen Geschichte mehr ausgesetzt war, das entstanđene radiogene Argon im Mineral verbleibt, was die Voraussetzung für die Interpretation des $\mathrm{K} / \mathrm{Ar}$ - 
Verhältnisses als ein geologisches Alter ist.

Mit der weiteren Verbesserung der Meßgenauigkeit durch die Einfuhrung der massenspektrometrischen Isotopenverdunnungsanalyse konnten auch kaliärmere Minerale, wie Hornblende und hinsichtlich der Argondiffusion dichte Gesteine als Ganzes, wie Basalt, mit Hilfe der Kali-Argon-Methode datiert werden. Im Gegensatz zu den anderen Methoden, die nur für die Datierung von magmatischen Gesteinen geeignet sind, können mit der K/Ar-Methode auch Glaukonite und damit unter bestimmten Voraussetzungen Sedimente datiert werden.

Mit diesen drei Methoden der radiometrischen Altersbestimmung, der $\mathrm{U} / \mathrm{Pb}$, der $\mathrm{Rb} / \mathrm{Sr}$ - und der $\mathrm{K} / \mathrm{Ar}-M e t h o d e$, stehen dem Geochronologen nunmehr verschiedene bewăhrte Verfahren der geologischen Zeitmessung zur Verfügung, die sich zum Teil gegenseitig ergänzen und überprüfen können, ob die Voraussetzungen für die Interpretation der erhaltenen MeBergebnisse, die primär zunächst nur analytisch erhaltene Verhältnisse von Isotopenhäufigkeiten sind, als geologische Alter gegeben sind.

Neben diesen Methoden werden in den folgenden Kapiteln noch

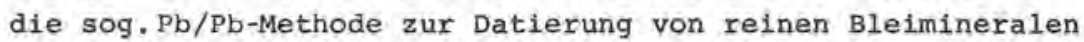
und die erst in jüngster zeit entwickelte Kernspurenmethode (fission track), die beide ebenfalls ein weites Anwendungsgebiet haben, behandelt. Einige weitere Verfahren, die zwar ebenfalls von wissenschaftlichem Interesse sind, die aber nur bel relativ seltenen Mineralen anwendbar sind, werden in dieser zusammenstellung nicht erwähnt.

Das vorliegende Buch soll dazu beitragen, dem interessierten Geowissenschaftler die wesentlichen Grundlagen der radiometrischen Methoden in der Geochronologie zu vermitteln und ihm durch diese Kenntnis einen nutzbringenden Einsatz dieser Verfahren ermöglichen, deren erfolgreiche Anwendung in der letzten Zeit in ständig zunehmendem MaBe wertvolle Informationen lieferte, die mit den klassischen Verfahren der Geologie nicht $z u$ erreichen sind. Dieses gilt besonders für den groBen Zeitabschnitt des Präkambriums, für den andere Methoden zur Erstellung einer Geochronologie kaum zur Verfügung stehen. 
Um das Thema dieses Heftes und damit dessen Umfang nicht zu weit zu fassen, wurde sich hier nur auf den physikalischmethodischen Teil der Verfahren der geologischen Zeitmessung beschränkt, und die Methoden der Datierung der jüngsten geologischen Geschlchte ( ${ }^{14} \mathrm{C}$-Methode) ausgeklamuert, da diese bereits in einem eigenen Heft (Clausthaler tektonische Hefte 11) behandelt worden sind. Die mineralogisch-petrographischen Aspekte der radiometrischen Altersbestimmung, die Auswahl geelgneter Proben und die Techniken der Mineralseparation werden als Ergänzung dieses Themas ebenfalls in einem gesonderten Heft behandelt werden. 


\section{DIE ISOTOPENVERDÜNNUNGSANALYSE}

(H. LENZ)

Bei allen Datierungsmethoden, die auf dem natürlichen radioaktiven Zerfall eines Elementes beruhen, besteht die Aufgabe, das Mengenverhältnis von zwei Isotopen zweier verschiedener Elemente mit einer Genauigkeit von etwa 1-3 $\%$ zu bestimmen. Es ist dies das Verhältnis des beim Zerfall entstehenden Tochterisotops $T$ zum Ausgangsisotop M. Mit dem gesuchten Alter steht es in der Bezlehung

$$
\frac{T}{M}=e^{+\lambda t}-1
$$

wobei $\mathrm{T}^{*}$ andeuten soll, $\mathrm{daB}$ es sich hierbei um ein radiogenes Isotop handelt. Ist das entstehende Tochterisotop bereits als natürliches Isotop in seinem Element vorhanden, so ist im Prin$z$ ip zunăchst eine Isotopenanalyse des entsprechenden Elementes erforderlich, um diesen "gewöhnlichen" Anteil anhand seiner bekannten Isotopenzusammensetzung eliminieren zu können.

Eine direkte Messung des Mengenverhältnisses zweier Isotope von zwei verschiedenen Elementen, was ja lediglich in die Altersbestimungsgleichung eingeht, ist nicht möglich. Es muß daher eine quantitative Analyse mit der geforderten Genauigkeit auf beide Elemente getrennt erfolgen, um dann damit das gesuchte Verhältnis bilden zu können.

Aus Gründen, die in der Probennahme selbst, der mineralogischen Aufbereitung und dem chemischen Aufschluß bedingt sind, wird man im allgemeinen z.B. bei einem Biotit mit einer Probenmenge von etwa $1 \mathrm{~g}$ arbeiten. Bei einem $\mathrm{Rb}-$ Gehalt von ca. $500 \mathrm{ppm}$ hat man also eine $\mathrm{Rb}-$ Menge von $500 \mu \mathrm{g}$. Hieraus ergibt sich bei einem Alter 300 ma eine Menge von ca. $0,5 \mu \mathrm{g}$ für das radiogene Sr.

Eine so geringe Menge läßt sich jedoch mit den herkömmlichen chemischen und physikalischen Analysenverfahren nicht mit der gewünschten Genauigkeit bestimmen.

*) ${ }_{\text {ma }}=$ Mililonen Jahre 
Hier bietet sich die massenspektrometrische Isotopenverdünnungsanalyse geradezu an, zumal zur Eliminierung des gewöhnlichen Anteils des Tochterelementes ohnehin eine Isotopenanalyse exforderlich ist. Diese Methode ist auf gasförmige, flüssige und feste Proben anwendbar und ist besonders für letztere bei der Bestimung sehr kleiner Gehalte allen anderen Methoden weit uberlegen. Sie wurde zuerst yon INGHRAM (1959) und Mitarbeitern zur quantitativen Analyse kleiner Mengen fester Stoffe verwendet und von ALDRICH (1953) bei Rb/Sr-Altersbestimungen benutzt.

Das Prinzip dieser Methode sei am Beispiel des Rb erläutert und in Abb. 2.-1 graphisch dargestellt. Bekanntlich hat das $\mathrm{Rb}$ in natürlicher Form zwei Isotope mit den Massenzahlen 85 und 87, die mengenmäBig im Verhältnis ${ }^{85} \mathrm{Rb} /{ }^{87} \mathrm{Rb}=2,593 \mathrm{zu}-$ einander stehen. $\mathrm{Zu}$ der $\mathrm{zu}$ analysierenden aufgeschlossenen Probe, die eine unbekannte Menge Rb mit natürlichem und daher bekannten Isotopenverhältnis besitat, wird eine genau bekannte Menge eines $\mathrm{Rb}$ hinzugefügt, das ein wesentlich anderes Isotopenverhältnis hat. Man verwendet in diesem speziellen Fall

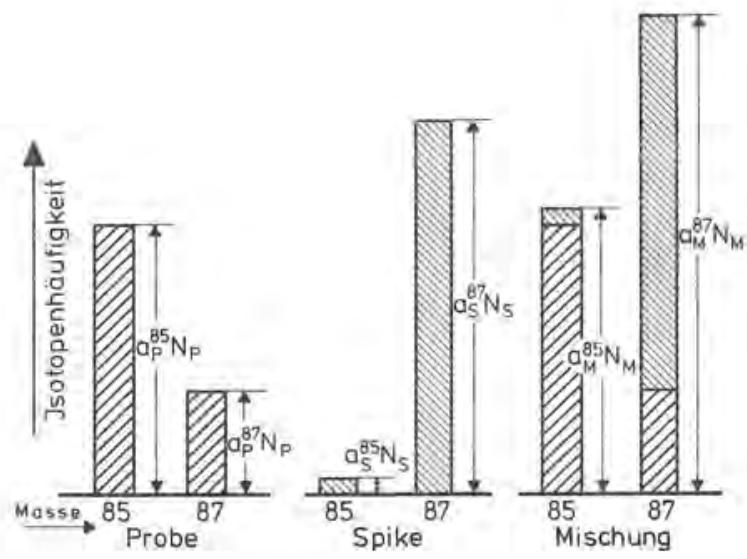

Abb. 2,-1: Prinzip der Isotopenverdünnungsanalyse 
meist fast reines ${ }^{87} \mathrm{Rb}$. Solche fast reinen oder hoch angereicherten Isotope können zum Beispiel vom Oak Ridge National Laboratory bezogen werden, und es hat sich für sie die Bezeichnung spike eingebürgert. Dieser spike wird mit dem zu analysierenden $\mathrm{Rb}$ in der Lösung völlig vermischt, das resultierende $\mathrm{Rb}$ der Mischung mit chemischen Methoden separiert und einer massenspektrometrischen Isotopenanalyse unterzogen. Dieses $\mathrm{Rb}$ hat nun infolge der Vereinigung von Proben- und Spike-Rb ein Isotopenverhältnis, welches zwischen den entsprechenden Verhältnissen der Komponenten liegt. Aus diesem Isotopenverhältnis der Mischung sowle dem der Komponenten und aus der bekannten Menge des zugegebenen Spikes läßt sich die gesuchte unbekannte Menge des Rubidiums in der Probe bestimun, wie eine rechnerische Behandlung dieses Vorganges weiter unten zeigen wird.

Die chemische Abtrennung des betreffenden Elementes nach der Durchmischung braucht nicht quantitativ zu sein, da lediglich das Isotopenverhältnis in die Berechnung der unbekannten Menge eingeht, Hierin liegt der Hauptvorteil der Isotopenverdünnungsanalyse, da gerade bei der Analyse kleiner Mengen die sonst erforderliche quantitative Abtrennung erhebliche Schwierigkeiten bereitet. Die Spikezugabe sollte natiurlich so frih wie möglich erfolgen, um diesen Vorteil voll auszunutzen.

Zur rechnerischen Behandlung der Isotopenverdúnnungsanalyse sei von der $\mathrm{Abb}, 2,-1$ ausgegangen. Die Höhe der dargestellten Balken soll hierin ein Maß für die Anzahl der Atome bzw. Mole sein. Im einzelnen sei

$$
\begin{aligned}
& \mathrm{N}_{\mathrm{p}}=\text { Anzahl der Rb-Atome der Probe } \\
& \mathrm{N}_{\mathrm{s}}=\text { Anzahl der Rb-Atome des Spikes } \\
& \mathrm{N}_{\mathrm{M}}=\text { Anzahl der Rb-Atome der Mischung. }
\end{aligned}
$$

Ferner seien ${ }^{85} \mathrm{a}_{\mathrm{p}} ;{ }^{85} \mathrm{a}_{\mathrm{s}} ;{ }^{85} \mathrm{a}_{\mathrm{M}}$ die relativen Anteile der Isotope der Masse 85 in Probe, Spike und Mischung und ${ }^{87} a_{p} ;{ }^{87} a_{s} ;{ }^{87} a_{M}$ die entsprechenden Anteile der Isotope der Masse 87. Definitions- 
gemäß gelten die Beziehungen

${ }^{85} a_{p}+{ }^{87} a_{p}=1 ; \quad 85 a_{s}+{ }^{87} a_{s}=1 ; \quad{ }^{85} a_{M}+{ }^{87} a_{M}=1$,

Für beide Massen läBt sich nun je eine Gleichung aufstellen, die die Bilanz der Atomzahlen beinhaltet.

$$
\begin{aligned}
& { }^{85} a_{p} N_{p}+{ }^{85} a_{s} N_{s}=85 a_{M} N_{M} \\
& 87 a_{p} N_{p}+87 a_{s} N_{s}=87 a_{M} N_{M}
\end{aligned}
$$

Die Division dieser beiden Gleichungen und die Einführung der Isotopenverhältnisse

$$
\alpha_{P}=\frac{85}{87 a_{p}} ; \quad \alpha_{s}=\frac{a_{s}}{87 a_{s}} ; \quad \alpha_{M}=\frac{a_{M}}{87 a_{M}}(2 .-3)
$$

ergibt schlieblich die gesuchte Beziehung

$$
N_{p}=N_{s} \frac{1+\alpha_{p}}{1+\alpha_{s}} \quad \frac{\alpha_{M}-\alpha_{s}}{\alpha_{p}-\alpha_{M}}
$$

Wie man erkennt, enthalt diese Gleichung außer der $z u$ bestimmenden Probenmenge nur noch die bekannte Menge des zugefügten Spikes und die bekannten bzw. meßbaren Isotopenverhältnisse.

Selbstverständlich läBt sich dieses Verfahren nur auf Elemente anwenden, die mindestens zwei Isotope besitzen und für die ein entsprechender spike erhältilch ist. Das ist für alle bei Datierungen interessierenden Elemente der Fall.

Hat ein Element mehr als zwei Isotope, und zwar n, so lassen sich n-1 Gleichungen analog $(2,-4)$ aufstellen, und man erhä1t n-1 voneinander unabhăngige Ergebnisse und auf diese Weise eine Kontrolle des Resultates. Die überzăhligen Gleichungen lassen sich aber auch dazu verwenden, evtl. Massendiskriminie- 
rungen im Massenspektrometer zu eliminieren.

Ebenso lassen sich aus z.B. zwel Gleichungen zwei unbekannte Komponenten bestimen, wenn deren Isotopenverhältnisse bekannt sind. Beim Sr kann man auf diese Weise das radiogene und das gewöhnliche $\mathrm{Sr}$ mit einer einzigen Messung analysieren, und man hat noch eine Gleichung für Korrekturzwecke frei, da das Sr vier Isotope, nämlich die mit den Massenzahlen 84,86 , 87 und 88 besitzt.

Es tut sich natürlich die Frage nach der Menge des zuzufügenden Spikes auf, und man wird diese Menge selbstverständlich so dimensionieren, daß das Ergebnis möglichst genau wird.

$\mathrm{Zu}$ diesem $\mathrm{Zweck}$ muß man eine ungefähre Vorstellung von der unbekannten Menge haben, was meistens auch aus geologischen Gesichtspunkten der Fall sein wird, Vielfach wird man auch vor der genauen Isotopenverdünnungsanalyse zur Probenauswahl eine Röntgenfluoreszenzanalyse durchführen, die dann einen guten Anhaltspunkt für die Spikedimensionierung gibt.

Hat man überhaupt keine Vorstellung von der zu analysierenden Menge, so wird man zunächst aufs geratewohl spiken und ein Ergebnis erhalten, das im allgemeinen mit einem groBen Fehler behaftet ist, wie später gezeigt wird. Anhand dieses Ergebnisses wird man dann eine günstige Spikemenge wählen.

Bei Messungen an gasförmigen Proben, z.B. Argon, wählt man die spikemenge meist so, daß das Isotopenverhältnis der Mischung immer annahernd gleich ist. Man erreicht hierdurch, daß eine Verfälschung des Ergebnisses durch einen evtl. Memory-Effekt nahezu ausgeschlossen ist.

Bei Feststoffmessungen wird man dagegen die Spikemenge so wählen, daß der Fehler des Ergebnisses möglichst klein wira. Zu diesem Zweck sei der Ausdruck für das Ergebnis dex Verdünnungsanalyse $(2,-4)$ einer Fehlerbetrachtung unterzogen. Faßt man die mit Meßfehlern behafteten Größen zusamen:

$$
N_{p}=N_{s} \cdot Q ; \quad Q=\frac{1+\alpha_{p}}{1+\alpha_{s}} \frac{\alpha_{M}-\alpha_{s}}{\alpha_{p}-\alpha_{M}}
$$


so ist also der Ausdruck Q, das Verhältnis von Proben- $z$ U Spikemenge, fehlermäBig zu betrachten.

Nach der Fehlerrechnung ergibt sich für den relativen Fehler von $Q$

$\left(\frac{\delta_{Q}}{Q}\right)^{2}=f_{p}^{2}\left(\frac{\delta p}{p}\right)^{2}+f_{s}^{2}\left(\frac{\delta s}{s}\right)^{2}+f_{M}^{2}\left(\frac{\delta_{M}}{M}\right)^{2}$

wobei $\frac{\delta_{p}}{p} ; \frac{\delta_{s}}{s} ; \frac{\delta_{M}}{M}$ die relativen Fehlex der Isotopenverhältnisse von Probe, Spike und Mischung sind.

Fïr die Fehlerfaktoren erhält man

$$
\begin{aligned}
& f_{p}=\frac{-\left(1+\alpha_{M}\right) \alpha_{p}}{\left(1+\alpha_{p}\right)\left(\alpha_{p}-\alpha_{M}\right)} \\
& f_{s}=\frac{-\left(1+\alpha_{M}\right) \alpha_{S}}{\left(1+\alpha_{S}\right)\left(\alpha_{M}-\alpha_{s}\right)} \\
& f_{M}=\frac{\left(\alpha_{p}-\alpha_{S}\right) \alpha_{M}}{\left(\alpha_{M}-\alpha_{S}\right)\left(\alpha_{p}-\alpha_{M}\right)}
\end{aligned}
$$

Mit der Beziehung

$$
\alpha_{M}=\frac{\alpha_{s}+\alpha_{p} \frac{1+\alpha_{s}}{1+\alpha_{p}} Q}{1+\frac{1+\alpha_{s}}{1+\alpha_{p}}} Q
$$

lassen sich die Fehlerfaktoren als Funktion von Q, dem Verhälthis von Probenmenge zu Spikemenge, berechnen. Eine graphische Darstellung dieser Fehlerfaktoren ist in Abb. 2. -2 gegeben, und zwar mit den $\mathrm{z}$ ahlenwerten

$$
\alpha_{\mathrm{p}}=2,593 \quad \text { und } \alpha_{\mathrm{s}}=0,007953
$$

für das normale $\mathrm{Rb}$ und einen angenommenen $\mathrm{Rb}$-Spike. 


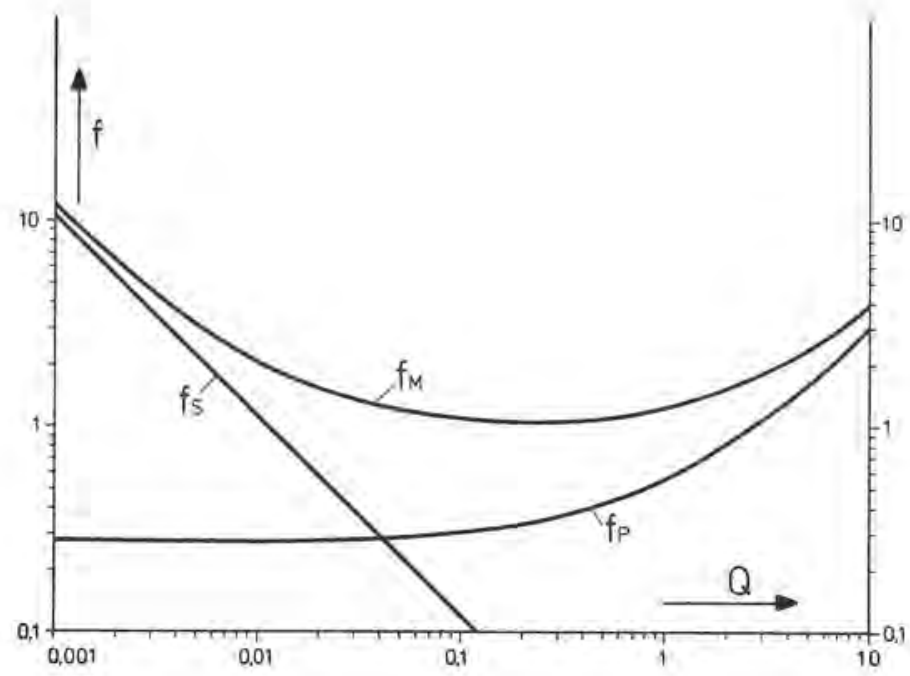

Abb. 2.-2: Fehlerfaktoren als Funktion des Probe-SpikeVerhälnisses $Q$

Der letztlich interessierende relative Fehler von $Q$ ist in Abb. 2.-3 graphisch dargestellt, und zwax unter der Annahme, daß die Meßfehler der Isotopenverhaltnisse alle $I$ o betragen. Wie man ersieht, besitzt dieser Fehler ein Minimum bei einem $Q$-Wert von 0,2 .

Es ist bei der vorstehenden Betrachtung nicht berücksichtigt worden, $d a \beta$ ein Isotopenverhältnis umso genauer $z u$ messen ist, je näher es bei 1 liegt. In das Diagramm ist daher noch das Isotopenverhältnis der Mischung $\alpha_{M}$ als Funktion von $Q$ eingetragen und es läßt sich ersehen, daß $\alpha_{M}$ den Wert 1 bei einem $Q$ von 2 erreicht. Um beiden Forderungen gerecht $z u$ werden, wird man die spikemenge so dimensionieren, daß $Q$ zwischen diesen beiden Werten von 0,2 und 2 liegt. Das flache Minimum des Fehlers von Q läBt einen ziemlich weiten spielraum zu. 


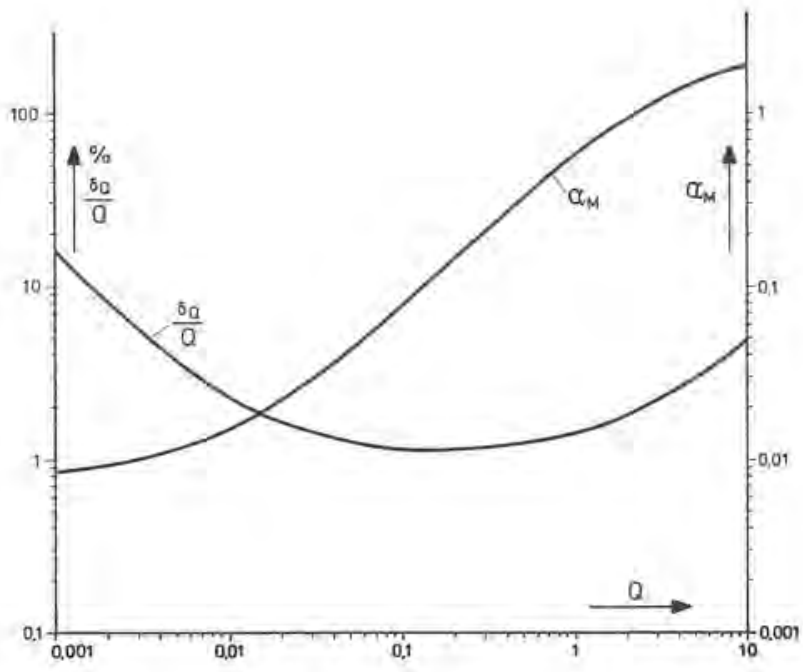

Abb. 2.-3: Relativer Fehler als Funktion von $Q$

\section{LITERATUR}

ALDRICH, T.L., DAVIS, G.L. \& DOAK, J.B.: Am. J. SCI. 251, S. $377,1953$.

INGHRAM, M.G.: Ann. Rev, Nuclear Sci, 4., S, 81, 1959. 


\section{DIE RUBIDIUM-STRONTIUM-METHODE}

\subsection{Einleitung}

Die Methode der Altersbestimmung mit Hilfe von natürlichen, langlebigen, radioaktiven Isotopen wird schon seit mehreren Jahrzehnten zur Datierung von Uranmineralen angewendet. Nach der Entdeckung der Radioaktivität des Isotops ${ }^{87} \mathrm{Rb}$ wurde die Möglichkelt, den zerfall des ${ }^{87} \mathrm{Rb}$ zum ${ }^{87} \mathrm{Sr}$ für aie Datierung von Gesteinen bzw. Rb-reichen Mineralen zu benutzen, mehrfach erwahnt (HAHN et al. 1937; STRASSMANN U. WALLIG 1938; MATTAUCH 1937).

Das Element $\mathrm{Rb}$ hat zwei Isotope: ${ }^{85} \mathrm{Rb}$ und ${ }^{87} \mathrm{Rb}$ mit einem Isotopenverhaltnis von ${ }^{85} \mathrm{Rb}:{ }^{87} \mathrm{Rb}=2.5995 \pm 0.0015$ (SHIELDS et al. 1963), das nach den Untersuchungen von SHIELDS et al. (1963) für alle natürlichen Vorkommen und Mineralalter als konstant angenommen werden darf. Das Isotop ${ }^{87} \mathrm{Rb}$ ist radioaktiv und zerfällt unter Emission eines B-Teilchens von $272 \mathrm{KeV}$ Maximalenergie zum ${ }^{87} \mathrm{Sr}$ mit einer Halbwertszeit von etwa $5 \times 10^{10} \mathrm{a}$. Eine Zusammenstellung von ALDRICH u. WETHERILL (1958) einer großen $\mathrm{Zahl}$ von Halbwertszeitbestimungen verschiedener Autoren zeigt erhebliche Diskrepanzen. Einige neuere Bestimungen sind in folgender Tabelle zusammengestellt.

HWz (in $10^{10} \mathrm{a}$ )
$4.6+0.5$
$4.3 \pm 0.3$
$5.0 \pm 0.2$
$5.07 \pm 0.2$
$4.7+0.1$
$4.7 \pm 0.05$
$4.72 \pm 0.04$

Methode

Autor
Fritze u. Straßmann

Huster

Aldrich et al.

Libby

Elynn, Glendenin

Glendenin

McMullen et al.
1956 1956 1956 1957 1959 1961 1966 
Für Altersbestimungen wird jetzt von vielen Autoren der Wert $4.7 \times 10^{10} \mathrm{a}\left(\lambda=1.47 \times 10^{-11} \mathrm{a}^{-1}\right)$ verwendet. Eine Reihe von amerikanischen und russischen Autoren bevorzugen jedoch weiterhin die Halbwertszeit von $5.0 \times 10^{10} \mathrm{Jahre}$, da häufig bei geologischen Proben mit diesem Wert eine bessere Ubereinstimmung mit anderen Datierungsmethoden erreicht wird. Alle neueren direkten Bestimunuen bestätigen aber den og. Wert von $4.7 \times 10^{10} \mathrm{a}$, wobei gänzlich verschiedene MeBmethoden angewendet worden sind. GLENDENIN hat eine Bestimung dex absoluten spezifischen Aktivität des Rubidiums vorgenommen, die eine gewisse Unslcherheit in der Extrapolation des gemessenen B-Spektrums bis zur Energie Null enthält. McMuLLEN et al. (1966) dagegen haben die durch Zerfall von ${ }^{87} \mathrm{Rb}$ innerhalb von sieben Jahren entstandene Menge ${ }^{87} \mathrm{Sr}$ massenspektrometrisch bestimmt und in fünf parallelen Versuchen Ergebnisse mit nur etwa 1 \& Streuung erhalten.

Aufgrund des oben Gesagten ist also beim Vergleich von Alterszahlen verschiedener Autoren darauf $\mathrm{zu}$ achten, mit welcher der beiden zurzeit gängigen Halbwertszeiten diese Alterswerte aus den analytischen Daten berechnet sind, und ggfs. alle Werte auf eine Halbwertszeit umzurechnen.

\subsection{Die Datierung von Mineralen}

Das Alter eines Minerals bzw. Gesteins ergibt sich nach $\mathrm{T}={ }^{87} \mathrm{Sr}$ und $\mathrm{M}={ }^{87} \mathrm{Rb}$ aus der Beziehung

$$
\frac{{ }^{87} \mathrm{Sr}}{87 \mathrm{Rb}}=\left(\mathrm{e}^{+\lambda t}-1\right) \approx \lambda t,
$$

wobei ${ }^{87} \mathrm{Sr}$ und ${ }^{87} \mathrm{Rb}$ die heutigen molaren Konzentrationen von radiogenem, d.h. aus dem Zerfall des ${ }^{87} \mathrm{Rb}$ entstandenen ${ }^{87} \mathrm{Sr}$ und ${ }^{87} \mathrm{Rb}$ sind. Die lineare Approximation des Exponentialausdruckes in obiger Formel ist wegen des sehr kleinen Wertes von $\lambda$ für nicht zu hohe Alter $\left(t<10^{9} \mathrm{a}\right)$ genugend genau. Die Genauigkeit der Datierung nach obiger Formel hängt also von der Genauigkeit der Bestimung des Gehaltes an radiogenem ${ }^{87} \mathrm{Sr}$ und $\mathrm{Rb} \mathrm{ab}$, wobei ersteres bei jüngeren Mineralen nur in sehr kleinen Konzentrationen (in Größenordnung von 1 $\mu \mathrm{g} / \mathrm{gMa}-$ terial) vorliegt. Die Bestimmung des $\mathrm{Sr}$ und $\mathrm{Rb}$ wird heute fast 
ausschlieBlich mit der Isotopenverdünungsmethode unter Verwendung von hochangereicherten ${ }^{84} \mathrm{Sr}$ - oder ${ }^{86} \mathrm{Sr}$-"Isotopenspikes" durchgeführt (ALDRICH 1956; HINTENBERGER 1960). SCHUMACHER (1956) hat mit diesen Verfahren $\mathrm{Rb}$ und Sr-Gehalte an den Steinmeteoriten "Forest-City" und "Pasamonte" und daraus Alter von $4.59 \pm 0.4 \times 10^{9}$ und $4.87 \pm 0.4 \times 10^{9} \mathrm{a}$ bestimmt. In gleicher Weise, aber mit wesentlich verbesserten Meßmethoden und Genauigkeiten von $\pm 0.1 \%$ im Isotopenverhältnis ${ }^{87} \mathrm{Sr} /{ }^{86} \mathrm{Sr}$ haben PAPANASTASSIOU et al. (1970) $\mathrm{Rb} / \mathrm{Sr}$-Datierungen an Mondgestein vom "Apollo 11"-Unternehmen datiert und hierbel Alter von $3.65 \times 10^{9}$ und $4.6 \times 10^{9}$ a ermittelt.

Die ersten Altersbestimmungen nach der $\mathrm{Rb} / \mathrm{S} r$-Methode sind vorwiegend an sehr $\mathrm{Rb}$-reichen und Sr-armen Mineralen (Lepidolith) durchgeführt worden, bei denen das vorhandene Sr fast ausschließlich aus radiogenem $\mathrm{Sr}$ besteht. Diese Minerale sind aber relativ selten, und die Rb/Sr-Methode hat erst ihre große Bedeutung gewonnen, als es möglich war, in magmatischen Gesteinen häufig vorkommende Minerale, wie Biotit, Muskowit, Kalifeldspäte oder Proben des gesamten Gesteins zu datieren. Diese Minerale enthalten außer dem durch radioaktiven Zerfall entstandenen ${ }^{87} \mathrm{Sr}$ auch gewöhnliches $\mathrm{Sr}$, das aufgrund seiner Isotopenzusammensetzung ${ }^{84} \mathrm{Sr}=0.56 \%$, ${ }^{86} \mathrm{Sr}=9.868,{ }^{87} \mathrm{Sr}=7.02 \%,{ }^{88} \mathrm{Sr}=82.56$ mit zu der totalen ${ }^{87}$ Sr-Konzentration beiträgt. Für den in die Formel eingehenden ${ }^{87} \mathrm{Sr}-$ Gehalt ist also die Differenz von totalem ${ }^{87} \mathrm{Sr}$ und Gehalt im yewöhnlichen ${ }^{87} \mathrm{~S} r$, der aus der relativen Häufigkeit eines der anderen Isotope, meist ${ }^{88} \mathrm{Sr}, \mathrm{zu}$ errechnen ist, einzusetzen. Es ist somit unmittelbar einzusehen, das die Bestimmung der ${ }^{87}$ Sr-Konzentration umso ungenauer wird, je mehr gewöhnliches Sr in der Probe vorhanden ist. Man ist daher bestrebt, Minerale für die Datierungen zu verwenden, die einen möglichst hohen Gehalt an radiogenem ${ }^{87} \mathrm{Sr}$ haben, also $\mathrm{Rb}$-reich sind und möglichst wenig gewöhnliches Sr enthalten. Solche Minerale, die auberdem auch noch in ausreichender Menge in den meisten magmatischen Gesteinen enthalten sein müssen, sind in erster Iinie die Glimuer. Die Gevinnung der 
reinen Biotit- und Muskowitfraktionen geschieht durch Trockenschütteltische (FAUL 1959), Siebung und Gewinnung geeigneter KorngröBenfraktionen, Trennung in Magnetscheidern und weitere Reinigungsprozesse, die in manchen Fallen durch ein manuelles Auslesen ( $z, B$, bei chloritisierten Biotiten) ergänzt werden müssen (vergl. auch E.JÄGER 1962). Auf diese Weise werden Mineralfraktionen höchster Reinheit gewonnen, z.B, Biotit besser als 99,8 \& Reinheit). Nach einem chemischen AufschluB werden die Elemente $\mathrm{Rb}$ und $\mathrm{Sr}$ mit H1lfe von Ionenaustauschkolonnen getrennt (SCHUMACHER 1956b; ALDRICH 1956) und durch Isotopenverdinnungsanalyse quantitativ bestimat. Einige Bearbeiter haben die Rb-Bestimmung auch mit der Neutronenaktivierungsanalyse durchgeführt (CABELL u. SMALES 1957).

Das aus dem Verhältnis ${ }^{87} \mathrm{Sr} /{ }^{87} \mathrm{Rb}$ errechnete "Modellalter" 1st unter folgenden Voraussetzungen als das wahre Alter des Minerals oder Gesteins anzusehen.

1. Die Isotopenzusammensetzung des bei der Mineral-oder Gesteinsbildung eingebauten Strontiums ist bekannt.

2. Vom Zeitpunkt der Kristallisation bis zur Probenentnahme ist das Mineral oder Gestein ein geschlossenes System gewesen, d.h. es ist während der geologischen Geschichte der Probe ke1n $\mathrm{Sr}$ oder $\mathrm{Rb}$ durch Sekundärprozesse, wie z.B. metamorphe Beanspruchung oder Auslaugung durch zirkulierende Lösungen zu- oder abgefüht worden.

ob diese Voraussetzungen für eine untersuchte probe erfültt sind oder nicht, kann in vieler Weise geprüft werden. Zeigen alle kogenetischen Mineralfraktionen einer Gesteinsprobe (Biotit, Muskowit, Feldspat usw.) das gleiche Modelialter wie die Gesamtgesteinsprobe und stimmt dieses Rb/Sr-Alter auch noch mit den nach der $\mathrm{K} / \mathrm{Ar}$-Methode bestimmten Altern der gleichen Mineralfraktionen liberein, so darf angenomen werden, daß dieses Alter den wahren Zeitpunkt der Bildung des Gesteins repräsentiert, besonders, wenn weitere Proben desselben Gesteinskörpers dieses Alter bestätigen. Häufig sind diese Voraussetzungen erfült. KULP et al. (1963) hat aus der Literatur eine Liste von etwa 150 konkoralanten Rb/Sr-Altern 
zusammengeste11t, der eine Reihe von 22 Proben mit diskordanten Altern gegenübersteht. Verschiedene Vorgänge können zur Ausbildung von diskordanten Alterswerten führen.

1. Das zur Zeit $t_{1}$ gebildete Gestein wird $z u$ einem späteren Zeitpunkt $t_{2}$ noch elnmal metamorph beansprucht, wobei sich die einzelnen Minerale neu bilden, aber kein Materialtransport uber größere als mikroskopische Entfernungen stattfindet. Eine Gesteinsprobe als Ganzes stellt in diesem Fall ein geschlossenes system dar, innerhalb dessen eine Neuverteilung von $\mathrm{Rb}$ und $\mathrm{Sr}$ auf die einzelnen Minerale stattgefunden hat. Man orhält Alterswerte, die alle größer als $t_{2}$ sind, die Minerale haben radiogenes Sr "ererbt", Die Datierung einer Probe des Gesamtgesteins liefert in diesem Fall das richtige Alter $t_{1}$ der Erstbildung des Gesteins, während Sr-reiche und $\mathrm{Rb}$-arme Minerale ein wesentlich höheres scheinbares Alter ergeben.

2. Das primär bei der Gesteinsbildung in die Minerale eingebaute "gewöhnliche strontium" hat bereits sog. "ererbtes" radiogenes Strontium aufgenommen, das ${ }^{87} \mathrm{Sr} /{ }^{86} \mathrm{Sr}-$ Verhältnis dieses Strontiums ist damit höher als der Normalwert von $0.708 \pm 0.03$ (HURLEY 1963; HART 1961). Das unter diesen Gegebenheiten mit dem Normalwert von 0.708 berechnete scheinbare Alter ist für alle Mineralfraktionen und für das Gesamtgestein zu hoch und zwar um so mehr, je kleiner kleiner das Verhälthis von RbGehalt $z u$ Gesamt-Sr-Gehalt ist.

3. Das zur zeit $t_{1}$ gebildete Gestein verliert ständig im Laufe seiner geologischen Geschichte oder zu einem späteren zeitpunkt $t_{2}$ durch starke metamorphe Beanspruchung oder geochemische Prozesse, wie Auslaugung von Verwitterungs lösungen und zirkulierenden wässern, teilweise aber nicht vollständig $\mathrm{Rb}$ oder $\mathrm{Sr}$, und auch eine Gesteinsprobe von 40 - $60 \mathrm{~kg}$ kann nicht mehr als geschlossenes System angesehen werden. Die scheinbaren Alter sind in Fall von $\mathrm{Rb}$-Verlusten $\mathrm{zu}$ hoch, bei Sr-Verlusten oder Rb-Zufuhr zu niedrig. Ein ähnliches Bild liefern auch die sich aus der K/Ar-Datierung ergebenden Alter. 
Für die pälle 1 und 2 kann bel einer genügenden Anzahl von Proben des gleichen Gesteins und verschiedenen Mineralfraktionen derselben probe unter bestimmten Voraussetzungen das wahre Alter der Bildung des Gesteins noch ermittelt werden, während im Falle eines offenen Systems (Fall 3) das wahre Alter meist nicht mehr angegeben werden kann.

\subsection{Die Isochronenmethode}

NICOLAYSON (1961) hat ein Verfahren der Auswertung von diskordanten Rb/Sr-Aitern entwickelt, das von LANPHERE et al. (1964) weiter ausgearbeitet wurde, mit dem fur die og. Fälle 1 und 2 eine Ermittlung des wirklichen Gesteinsalters unter gewissen Voraussetzungen möglich ist. Schreibt man die Altersformel, bezogen auf das stabile sr-Isotop ${ }^{86} \mathrm{Sr}$ in folgender Form:

$$
\begin{aligned}
& \frac{{ }^{87} \mathrm{Sr}}{{ }^{86} \mathrm{Sr}}=\left(\frac{87 \mathrm{Sr}}{{ }^{86} \mathrm{Sr}}\right)+\frac{87 \mathrm{Rb}}{{ }^{86} \mathrm{Sr}}\left(e^{+\lambda t}-1\right) \text { oder } \\
& y=y_{0}+a(t) \cdot x ; \quad \text { mit } \frac{87 \mathrm{Sr}}{{ }^{86} \mathrm{Sr}}=y ; \frac{{ }^{87} \mathrm{Rb}}{{ }^{86} \mathrm{Sr}}=\mathrm{x},
\end{aligned}
$$

so ist das die Gleichung einer Geraden im $\frac{87 \mathrm{Sr}}{86_{\mathrm{Sr}}} / \frac{87 \mathrm{Rb}}{86 \mathrm{Sr}}-$ System mit dem Anfangswert $\left(\frac{87 \mathrm{Sr}}{{ }^{86} \mathrm{Sr}}{ }_{0}\right.$, das ist das

${ }^{87} \mathrm{Sr} /{ }^{86} \mathrm{Sr}$-Isotopenverhältnis zum Zeitpunkt der Bildung des Minerals, und der steigung $\left(e^{+\lambda t}-I\right)$.

Die Anderung der $\frac{{ }^{87} \mathrm{Rb}}{{ }^{86} \mathrm{Sr}}$ und $\frac{{ }^{87} \mathrm{Sr}}{{ }^{86} \mathrm{Sr}}$ Verhältnisse mit der zeit $t$ sind aus den Entwicklungslinien ( $\mathrm{Abb}, 3 .-1$ ) zu ersehen. Zum Zeitpunkt $t_{0}$, der Bildung des Gesteins, hat das in allen Mineralen mit verschiedenem $\mathrm{Rb}$-Gehalt vorhandene Strontium das Isotopenverhältnis $\mathrm{y}_{0}=\left(\frac{{ }^{87} \mathrm{Sr}}{{ }^{86} \mathrm{Sr}_{0}}\right)$.

Die ${ }^{87} \mathrm{Sr}$ und ${ }^{87} \mathrm{Rb}-$ Gehalte in den einzelnen Mineralen ändern sich mit fortlaufender zeit 


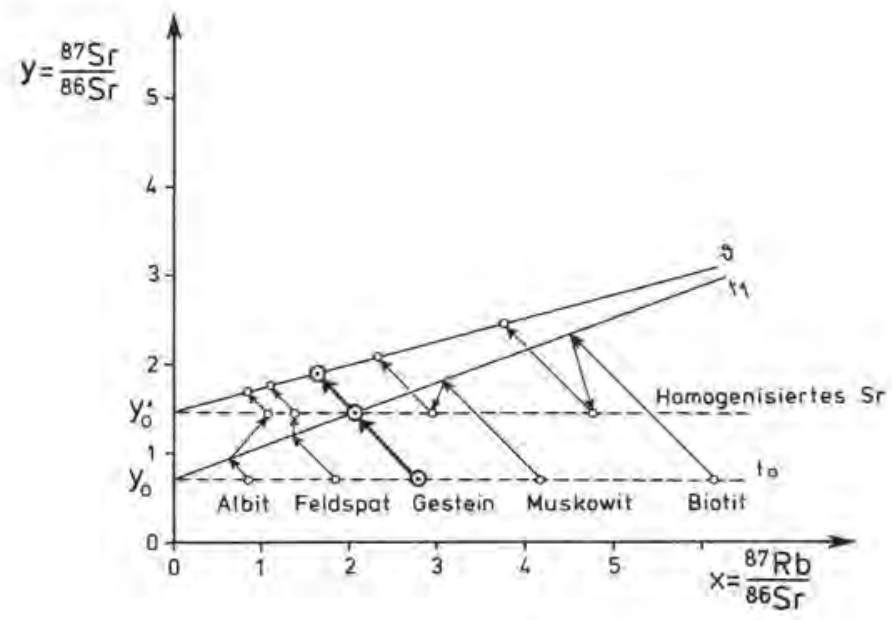

Abb. 3. - 1: Entwicklungsdiagramn der ${ }^{87} \mathrm{Sr} /{ }^{86} \mathrm{Sr}$ und ${ }^{87} \mathrm{Rb} /{ }^{86} \mathrm{Sr}$-Verhâltnisse

$\frac{{ }^{87} \mathrm{Sr}}{{ }^{86} \mathrm{Sr}}=\left(\frac{{ }^{87} \mathrm{Sr}}{{ }^{86}{ }_{\mathrm{Sr}}}\right)_{0}+\left(\frac{{ }^{87} \mathrm{Rb}}{\left.{ }^{86}\right)_{\mathrm{Sr}}},\left(1-\mathrm{e}^{-\lambda t}\right):\right.$

$\frac{{ }^{87} \mathrm{Rb}}{{ }^{86} \mathrm{Sr}}=\left(\frac{{ }^{87} \mathrm{Rb}}{{ }^{86} \mathrm{Sr}}, \mathrm{e}^{-\lambda t}\right.$

und die steigung $\frac{\text { d } \frac{{ }^{87} \mathrm{Sr}}{{ }^{86} \mathrm{Sr}}}{\left.\text { d } \frac{{ }^{87} \mathrm{Rb}}{86 \mathrm{Sr}}\right)}=-1$

ist unabhängig vom $\mathrm{Rb}-$ Gehalt und der zeit $t$.

Mit einfachen Worten heißt das: Jedes zerfallende ${ }^{87} \mathrm{Rb}$-Atom wird ein ${ }^{87} \mathrm{Sr}$-Atom, also jeder Schritt auf der ${ }^{87} \mathrm{Rb} /{ }^{86} \mathrm{Sr}$-Achse rückwärts (in negative Richtung) ist gleichzeitig auf der ${ }^{87} \mathrm{Sr} /{ }^{86} \mathrm{Sr}-\mathrm{Achse}$ ein Schritt vorwärts (in positive Richtung). 
Die einzelnen Mineralkomponenten entwickeln sich also mit fortlaufenden $t$ in $\frac{{ }^{87} \mathrm{Sr}}{{ }^{86} \mathrm{Sr}} / \frac{87 \mathrm{Rb}}{86} \mathrm{Sr}$ - Diagramn auf Linien mit der Steigung -1 , Zum Zeitpunkt $t_{1}$ liegen die Endpunkte dieser Entwicklungslinien auf einer Geraden.

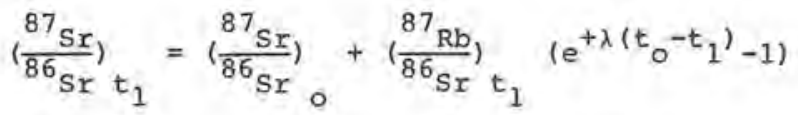

Zu diesem Zeitpunkt trete eine lokale Homogenisierung des Gesteins, z.B. durch Metamorphose ein, die sich in einer Auflösung der bestehenden Minerale, aber ohne einen Mineraltransport über nennenswerte Entfernungen auswirkt, so daß eine Gesteinsprobe als geschlossenes system angesehen werden kann. Bei diesem ProzeB werden $\mathrm{Sr}$ und $\mathrm{Rb}$ weder entfernt noch zugefügt, sondern nur neu vertelit. Das "gewöhnliche Strontium" in allen Mineralen, die sich zu diesem zeitpunkt neu gebildet haben, hat ein 87/86-Isotopenverhältnis, das dem Durchschnittswert der gesamten Gesteinsprobe zur zeit $t_{I}$ entspricht. Die ${ }^{87} \mathrm{Sr} /{ }^{86} \mathrm{Sr}$-Werte für alle Minerale sind somit gleich dem des

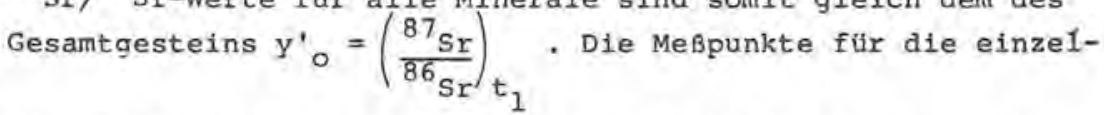

Minerale werden in dem Diagramm, also von der Isochrone $t_{1}$ in elner von den $\mathrm{Rb}$ - und Sr-Gehalten der neu gebildeten Minerale abhängenden Richtung auf eine horizontale neue Basislinie, die durch den unverschobenen Diagrammunkt des Gesamtgesteins geht, hin verschoben. Der ${ }^{87}$ Sr-Gehalt in den einzelnen Mineralkomponenten nimmt von diesem zeitpunkt an wieder entsprechend dem Rb-Gehalt mit der zeit zu, d.h. die den einzelnen Mineralkomponenten entsprechenden Punkte bewegen sich wieder entlang der Entwicklungslinie mit der steigung -1 und liegen zum heutigen Zeitpunkt auf einer neuen Isochrone

$$
\frac{{ }^{87} \mathrm{Sr}}{{ }^{86} \mathrm{Sr}}=\left(\frac{87 \mathrm{Sr}}{{ }^{86} \mathrm{Sr}_{1} t_{1}}+\frac{87 \mathrm{Rb}}{{ }^{86} \mathrm{Sr}_{\mathrm{r}}}\left(\mathrm{e}^{\left.+\lambda t_{1}-1\right)}\right.\right.
$$


die bei dem erhöhten Wert $y^{\prime}{ }_{0}=\left(\frac{87 \mathrm{Sr}}{{ }^{86} \mathrm{Sr} t_{1}}\right.$ die ordinate schneidet und eine Steigung hat, die durch das Alter $t_{1}$ der Metamorphose gegeben ist.

Ist ein. Gesteinskomplex vor $t_{0}$ Jahren gebildet und vor $t_{1}$ Jahren einer Metamorphose unterworfen worden, so liegen bei mehreren Gesteinsproben, die sich im Rb-Gehalt unterscheiden, die Gesamtgesteinsmeßpunkte auf einer Isochrone, deren Steigung $\tan \alpha=\left(e^{+\lambda t} 0-1\right)$ ist, während die einzelnen Mineralphasen jeder Probe wiederum auf Geraden liegen, die alle die gleiche steigung $\tan \alpha^{\prime}=\left(e^{+\lambda t_{1}}-1\right)$ aber verschiedene $\left(\frac{87 \mathrm{Sr}}{{ }^{86} \mathrm{Sr}}\right)$-werte $\mathrm{B}_{1}$ haben, je nach dem ${ }^{87} \mathrm{Rb} /{ }^{86} \mathrm{Sr}$-Verhältnis der diesen Mineralproben zugehörigen Ganzgesteinsprobe (Abb. 3. -2).

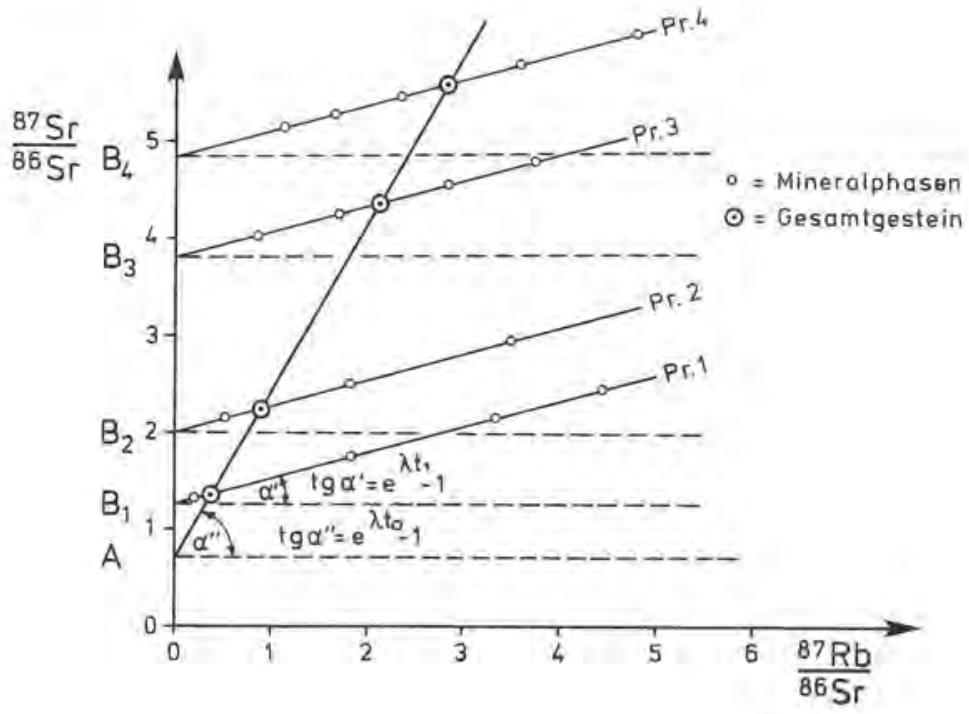

Abb. 3.-2: Prinzip der Gesteins- und Mineralisochronen 
Eine Reihe von $\mathrm{Rb} / \mathrm{Sr}$-untersuchungen haben dieses $\mathrm{Bild}$ bestätigt. JÄGER und NIGGLI (1964) konnten in einer Untersuchung des Rotondo-Granits zeigen, daB fünf verschiedene Gesteinsproben eine Isochrone mit einer dem Alter von 260 ma entsprechenden Stelgung und einem Anfangs- ${ }^{87} \mathrm{Sr} /{ }^{86} \mathrm{Sr}$-Verhältnis von 0.687 bis 0.700 liefern, während die Mineralphasen Biotit, Kalifeldspat, Gestein und Albit wiederum auf einer Geraden entsprechend einem Alter von 13 ma und einem Anfangswert von 0.905 (Abb. 3.-3) 1iegen. Ahnliche detaillierte Isotopenana1ysen wurden auch von LONG (1964) an einem Granit aus schottland durchgeführt. Ebenso haben LANPHERE et al. (1964) dieses Verfahren in Verbindung mit umfangreichen weiteren Isotopendatierungen nach der $\mathrm{Pb} / \mathrm{U}$ - und der $\mathrm{K} / \mathrm{Ar}$-Methode angewendet, um die komplizierte geologische Geschichte eines Gneiskomplexes in Ost-Kalifornien zu klären.

Das $\mathrm{Rb} / \mathrm{Sr}$-Isochronenverfahren hat sich heute $\mathrm{zu}$ einer Standardmethode für die Datierung von Gesteinen entwickelt. Für die Behandjung eines Datierungsproblems ist eine Anzahl von Proben, - möglichst sechs oder mehr - mit möglichst unter-

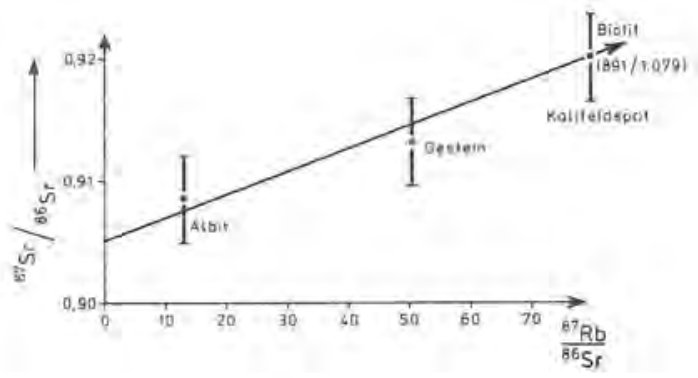

Abb, 3.-3: Beispiel einer Mineralisochrone des Rotondo-Granits (nach E.JAGER U. E.NIGGLI 1964) 
schiedlichen Rb/Sr-Verhältnissen erforderlich. Es wird daher zweckmäßigerweise im Gelände eine größere Probenzahl genommen, aus der mit Hilfe einer analytischen Schnellmethode, (z.B. durch Röntgenfluoreszenzanalyse (RFA)) hinsichtlich des $\mathrm{Rb}$ - und $\mathrm{Sr}-$ Gehaltes geeignete Proben für die weitere Bearbeitung ausgesucht werden. Die relative Genauigkeit, mit der die Steigung der Isochrone und damit das Alter der probenserie bestimmt werden kann, hängt von den zwei MeBgrößen ab: dem ${ }^{87} \mathrm{Rb} /{ }^{86} \mathrm{Sr}$-Verhältnis und dem ${ }^{87} \mathrm{Sr} /{ }^{86} \mathrm{Sr}$-Verhältnis. Bei kleinen Altern oder niedrigen ${ }^{87} \mathrm{Rb} /{ }^{86} \mathrm{Sr}$-Werten spielt die Genauigkeit des ${ }^{87} \mathrm{Sr} /{ }^{86} \mathrm{Sr}$-Verhältnisses die entscheidende Rolle. Bisher war diese Datierungsmethode aus diesen Gründen auf saure Intrusivgesteine, wie z.B. Granite, beschrănkt, und für basische Gesteine konnten lediglich Mineraldatierungen vorgenommen werden, mit denen, wie oben schon gesagt wurde, eine Bestimung des Intrusionsalters nicht immer möglich ist. Erst in jüngster Zeit, nachdem es mit der Weiterentwicklung der massenspektrometrischen Meßverfahren und der Einführung der digitalen Datenausgabe möglich wurde, dieses Isotopenverhältnis auf 0.01 o genau zu messen, kann die Anwendung dieser Datierungsmethode auch auf basische Gesteine ausgedehnt werden.

Rechenverfahren, durch eine 1 ineare Anordnung von Meßpunkten eine optimale Gerade nach der Methode der kleinsten Quadrate zu legen, sind von BROOKS et al. (1969); MCINTYRE et al (1966) und YORK (1966) ausgearbeitet worden. Mit den in diesen Arbeiten angegebenen Rechenprogrammen können aus den Meßwerten nebst ihren Fehlergrenzen die Werte fur die Steigung der Isochronen und den Anfangswert einschließlich deren Fehlergrenzen berechnet werden.

\subsection{Das Compston-Jeffery-Verfahren}

Auf eine etwas andere Weise haben COMPSTON u. JEFFERY (1961) aus den ${ }^{87} \mathrm{Sr} /{ }^{86} \mathrm{Sr}$ - und ${ }^{87} \mathrm{Rb} /{ }^{86} \mathrm{Sr}$-Werten die Mineralphasen des Metamorphosealters unter der Voraussetzung einer vollständigen Homogenisierung in situ ermittelt. 
Die Gleichung

$$
\theta=\left(e^{\lambda t}-1\right)=\frac{\frac{87 S r}{86}-\left(\frac{87 S r}{86}\right)}{\frac{87 \mathrm{Rb}}{{ }^{86} \mathrm{Sr}}}=\left(y-y_{0}\right) \frac{1}{x}
$$

oder

$$
\theta=(y-n) \frac{1}{x}
$$

stellt eine Gerade im $\theta, n$-Diagramm dar, wenn der Anfangs $\frac{{ }^{87} \mathrm{Sr}}{{ }^{86} \mathrm{Sr}}$-Wert $\mathrm{y}_{0}$ bei durch die Messung festgelegten $y$ und $x$ Werten als Variable $n$ aufgefaßt wird (Abb. 3,-4). Falls alle Mineralphasen das gleiche Alter haben, so scheiden sich die diesen Phasen im Diagramm entsprechenden Geraden in einem Punkt ${ }^{\circ}{ }_{0} y_{0}$ der dem Alter der letzten totalen Homogenisierung und dem ursprünglichen ${ }^{87} \mathrm{Sr} /{ }^{86} \mathrm{Sr}$-Wert $\mathrm{y}_{0}$ entspricht. Hat das Gestein zu einem späteren zeitpunkt $\theta^{\prime}$ nach der Erstbildung (Alter $\theta_{0}$ ) eine Homogenisierung $z . B$. durch Metamorphose durchgemacht, so schneiden sich die durch

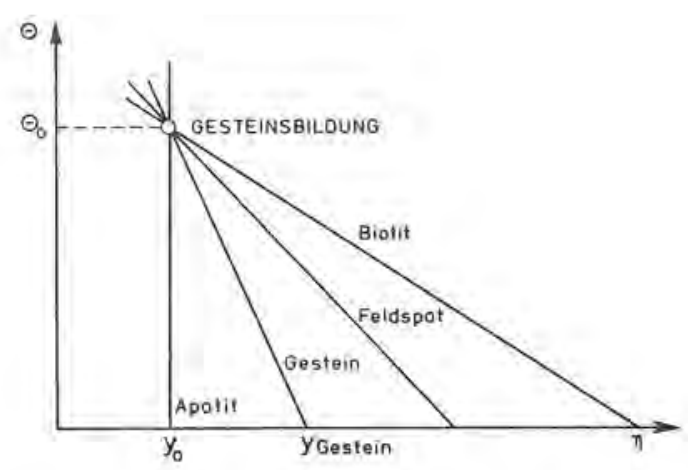

Abb. 3. -4 : Compston-Jeffery-Diagramm im Einstufenfall 
die für die einzelnen Mineralphasen erhaltenen $\mathrm{y}$ und $\mathrm{x}$ Werte festgelegten Geraden ebenfalls in einem Punkt $\theta$ ', $y_{0}^{\prime}$ wobei $Y_{0}^{\prime}$ : ist. Der Schnittpunkt der Gesteinsgeraden mit der Geraden $y=y_{0}$ oder der Schnittpunkt mehrerer Gesteinsgeraden liefert dann das Alter $\theta_{0}$ der Gesteinserstbildung. Die Entwicklungslinien in $\mathrm{Abb}$. 3.-5 beginnen alle im Punkt $\theta_{0}$, $y_{0}$, und zur Zeit $\theta$ '。 haben die $y$-Werte der einzelnen Mineralphasen die durch die Schnittpunkte ihrer Entwicklungslinien mit der Horizontalen $\theta=\theta^{\prime}$, festgelegten Werte angenommen. Durch die Homogenisierung werden dann alle y zu $y^{\prime} 0^{\prime}$ und die Entwicklungslinien beginnen erneut im Punkt $\theta^{\prime} 0^{\prime}{ }_{87} y^{\prime} 0^{\circ}$. Die Auswirkungen weiterer Vorgänge, wie partielle ${ }^{87} \mathrm{Sr}$ oder ${ }^{86} \mathrm{Sr}$ Umlagerung, Kontamination nach der Probennahme, ein oder mehrere Metamorphosen mit Homogenisierungen usw. sind von RILEY $u$. COMPSTON (1962) in einer umfangreichen theoretischen Analyse diskutiert worden.

In einigen Fällen sind mit dem Verfahren von COMPSTON bestimute Prozesse, die zu diskordanten Mineralaltern führen, besser

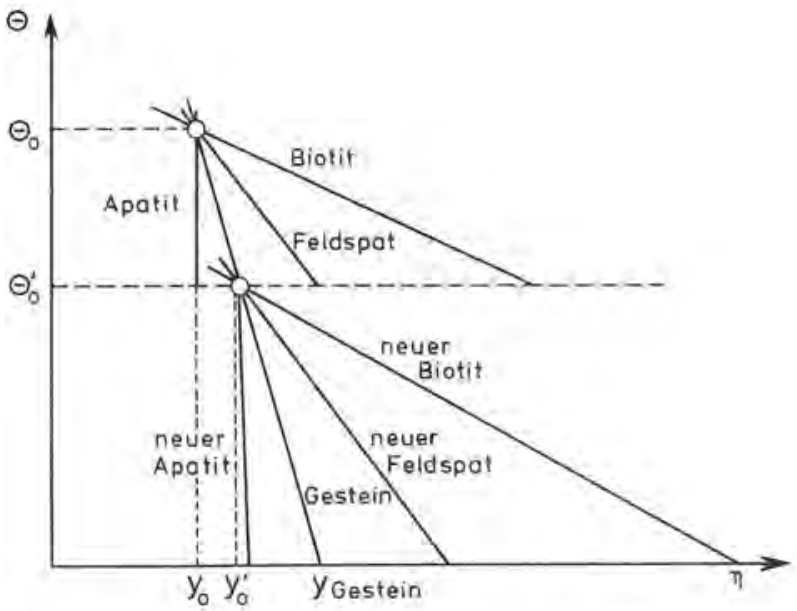

Abb, 3.-5: Compston-Jeffery-Diagramm im Zweistufenfall 
zu erkennen als mit der Isochronendarstellung. Uberwiegend wird aber die letztere verwendet, da außer der besseren Anschaulichkeit sie auch für die statistisch rechnerische Datenverarbeitung besser geeignet ist. Es muB aber darauf hingewiesen werden, daß selbst bei nach geologisch und petrographisch einwandfreien Gesichtspunkten entnommene Probenserien nicht immer für die Mineral- oder Ganzgesteinsisochronen oder in den COMPSTON'schen Diagrammen so klar interpretierbare Bilder gewonnen werden. Hat z.B. bei einer späteren sekundären Beanspruchung, wie eine schwache Metamorphose, keine vollständige Homogenisierung der $\mathrm{Rb}$ und $\mathrm{Sr} \mathrm{zW}$ schen den Mineralen stattgefunden, und diese Elemente sind nur z.T. ausgetauscht worden, so liefern die Minerale keine echte Isochrone mehr; sie streuen in dem NICOLAYSEN'schen Isochronendiagramm zwischen den dem Primäralter to und dem Sekundäralter $t_{1}$ entsprechenden Isochronen. Auch für eine Ganzgesteinsisochrone sind die Voraussetzungen nicht mehr erfüllt, wenn eine der Probengröße (meist 30 bis $50 \mathrm{~kg}$ ) entsprechende Gesteinsmenge hinsichtlich der Elemente $\mathrm{Rb}$ und $\mathrm{Sr}$ nicht mehr als geschlossenes System angesehen werden kann, und chemische Lösungsvorgänge diese Elemente uber größere Entfernungen transportiert haben. Auch bei einer zufälifgen mehr oder weniger linearen Anordnung der MeBpunkte sind Streuungen der Mespunkte um diese "Pseudoisochrone", die die analytischen Fehlergrenzen uberschreiten, starke Hinweise darauf, daB man es hier nicht mit einer echten Isochrone, aus der ein verläBlicher Alterswert bestimnt werden kann, zu tun hat. Wertvolle Hilfe leisten hier die Ergebnisse anderer Datierungsmethoden, KULP et al. (1963) haben z.B, gezeigt, daB bel Verlusten von Kalium, Rubidium und Strontium durch von Verwitterungslösungen bedingte Auslaugung bei vielen Mineralen das $\mathrm{K} / \mathrm{Ar}-" \mathrm{Alter}$ " verhältnismäBig wenig verändert wird, wăhrend das Rb/Sr-"Alter" sehr stark beeinfluBt wird. 
LITERATUR

ALDRICH et al.: Phys. Rev. 103, 1045, 1956.

ALDRICH, L.T. \& WETHERILI, G.W.: Geochronology by radioactive Decay. - Annual Rev. Nucl, Sc1. 8, 257, 1958.

ALDRICH, I.T.: Measurement of Radioactive Ages of Rocks. Science 123, 871-875, 1956.

CABELL, M.J, \& SMALES, A.A.: The Determination of Rubidium and Caesium in Rocks, Minerals and Meteorites by Neutronactivationanalysis. - Analyst 82, $390-906,1957$.

COMPSTON, W. \& JEFFERY, P.M.: Metamorphic Chronology by the Rubidium-Strontium-Method. - Anal. N.Y.AC. Sci. 91, Art. 2., 185-191, 1961.

FAUL, H. \& DAVIS, G.L.: Mineralseparation with asymetric vibrators. - Am. Min. 44, 1076-1082, 1959.

FAUL, H. \& JAGER, E.; Rb-Sr age determinations on micas and total rocks from the alps. - J. Geoph. Res. $67,5285-5293,1962$.

FLYNN, K.F. \& GLENDENIN, L.E.: Half-Life and Beta-spectrum of $\mathrm{Rb}^{87}$. - Phys. Rev. 116, 744-748, 1958 ,

FRITZE, K. \& STRASSMANN, F.: Indirekte Bestimung der Halbwertszeit des Rubidiums. - Z, Naturforsch. 11a, 277-280, 1956.

GLENDENIN, L.E.: Present Status of the Decay Constants. Anal. N.Y. Ac. Sci. Vol. II, Art. 2,, 166-180, 1961.

HAHN, O., STRASSMANN, E. \& WALIIG, E.: Herstellung wägbarer

Mengen des Strontiumsisotops ${ }^{87} \mathrm{Sr}$ als Umwandlungsprodukt des Rubidiums aus einem kanachitischen Glimmer. - Naturw. 25, 189, 1937.

HART, St.R.: Mineral Ages and Metamorphism, - Anal, N.Y. AC. Sci. 91, Art. 2., 192-198, 1961.

HINTERBERGER, H.: Die Rubidium-Strontium-Methode. - Geol. Rundschau 42, 197, 1960.

HURLEY, P.M., FAIRBAIRN, H.W., FAURE, G. \& PINSON, W.H.: New Approaches to Geochronology by Strontium Isotope Variations in whole Rocks. - Radioactive Dating: STI/PUB/68, 201-218, 1963, IAEA, Wien.

HUSTER, E.: A Redetermination of the Life of ${ }^{87} \mathrm{Rb}$. Nuc1. Sc. Ser. Rep. No. 19, 195, 1956. 
JKGER, E, \& NIGGLI, E.; Rubidium-Strontium-Isotopenanalysen an Mineralen und Gesteinen des Rotondogranites und ihre geologische Interpretation. Schweiz. Minera1. Petrogr. Mitt. 44, 61-81, 1964 .

KUIP, J.I. \& ENGELS, J.: Discordances in $\mathrm{K} / \mathrm{Ar}$ and $\mathrm{Rb} / \mathrm{Sr}$ Isotopic Ages. - Radioactive Dating STI/PUB/68, 219-238, 1963, IAEA, Wien,

I.AUPHERE, M.A., WASSERBURG, G.J.F., ALBEE \& TILTON, G.R.: Redistribution of Strontium and Rubidium Isotopes during metamorphism world beater complex, panamint range, California. Isotopic and Cosmic chemistry by MILLER, WASSERBURG, North Holland, 269-320, 1964.

LIBBY, W,F.: Simple Absolute Measurement Technique for Beta Radioactivity, Application to Naturally Radioactive Rubidium, - Anal. Chem, 29, No. 11, 1566, 1957.

MATTAUCH, J.: Das Paar ${ }^{87} \mathrm{Rb}-{ }^{87} \mathrm{SI}$ und die isobaren Regel. Naturw. $25,189,1937$.

MCINTYRE, G.A., BROOKS, C., COMPSTON, W. \& TUREK, A. : The Statistical Assesment of Rb-Sr Isochrons. J. Geophysical Research, Vol. 71, No. 22, 5459-5468, 1966.

MCMULLEN, C.C., FRITZE, K. \& TOMLINSON, R.H.: The Half Life of Rubidium-87. - Can, J. Phys. 44, 3033-3038, 1966 .

NICOLAYSEN, L.O.: Graphic Interpretation of discordant age measurements on metamorphic rocks. - Anal. N.Y. Ac. Sci. Vol. 91, Art. 2., 198-206, 1961.

RILEY, G.H. \& COMPSTON, W.: Theoretical and technical aspects of $\mathrm{Rb}-\mathrm{Sr}$ geochronology. - Geoch. Cosmochim. Acta 26, 1255-1281, 1962 .

SCHUMACHER, E.: Isollerung von $\mathrm{K}, \mathrm{Rb}, \mathrm{Sr}, \mathrm{Ba}$ und seltenen Erden aus Steinmeteoriten. - Helv. Chim. Acta 39, 531-537, 1956 .

- - - Altersbestimmung von Steinmeteoriten nach der $\mathrm{Rb}-\mathrm{Sr}$-Methode. - Z,f. Naturf. 11a, 206-212, 1956.

SHIELDS, W.R., GARNER, E.I., HEDGE, C.E. \& GOLDICH, S.S.: Survey of ${ }^{85} \mathrm{Rb} /{ }^{87} \mathrm{Rb}$ Ratio in Minerals. J. Geoph. Res. 68, 2331-2334, 1963.

STRASSMANN, F. \& WALlig, E.: Ber. Dtsch. chem. Ges. 71, 1, 1938. YORK, D.: Leastsquares fitting of a straight line. - Can. J. Bhys. 44, 1079-1086, 1966. 
4.-1 Isotope des Kaliums, Konstanten und Forme In

Ein weiteres, in der Natur häufig vorkommendes, langlebiges radioaktives Isotop ist das $40_{K}$. Nach NIER (1950) hat das Element Kalium folgende Isotopenhäufigkeiten:

$$
{ }^{39} \mathrm{~K}=93.088 ; \quad{ }^{40} \mathrm{~K}=0.0119 \% ; \quad 41_{\mathrm{K}}=6,918 .
$$

Das Zerfallsschema (Abb, 2,2,-1) des ${ }^{40} \mathrm{~K}$ zeigt einen B-Zerfall zum ${ }^{40} \mathrm{Ca}$ und einen $\mathrm{K}-$ Einfang mit anschließender $\mathrm{Y}$-Emission zum $40_{\mathrm{A} \text {. }}$

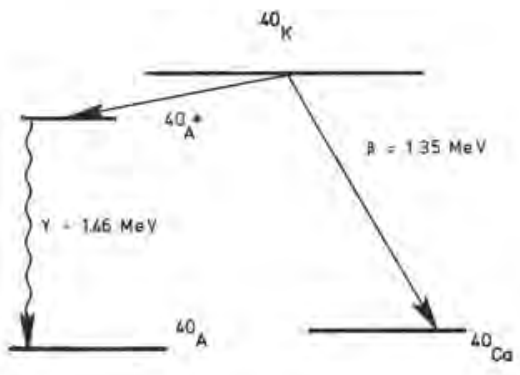

Nach GLENDENIN (1961) be-

trägt die spezifische B-Aktivität des Kaliums

$$
28.2 \pm 0.3\left(\frac{\mathrm{dpm}}{\mathrm{gK}}\right)
$$

woraus eine B-Halbwertszeit $T_{B}=(1.42 \pm 0.02) \times 10^{9} \mathrm{a}$ und
$\lambda_{\beta}=(4.72 \pm 0.05) \times 10^{-10} \mathrm{a}^{-1}$

Abb.4.-1: Das ${ }^{40} \mathrm{~K}-\mathrm{Zerfalls}$ schema

Mit dem verzweigungsverhältnis $\lambda_{\kappa}: \lambda_{\beta}=0.11 \pm 0.02$ erhält man für die $\mathrm{K}$-Einfang-Zerfallskonstante $\lambda_{\mathrm{K}}=0.577^{-} \times 10^{-10} \mathrm{a}^{-1}$ und für die totale Halbwertszeit $T=(1.26 \pm 0.04) \times 10^{9} \mathrm{a}$. Somit ergeben sich für Kaliuminerale prinzipiell zwei Datierungsmöglichkeiten, nämlich

$$
\frac{{ }^{40} \mathrm{Ca}}{4 O_{K}}=\frac{\lambda_{B}}{\lambda_{B}+\lambda_{K}}\left(e^{+\lambda t}-1\right)
$$

und

$$
\frac{4 O_{A}}{4 O_{K}}=\frac{\lambda_{K}}{\lambda_{B}+\lambda_{K}}\left(e^{+\lambda t}-1\right)
$$


Die Ca/K-Methode ist nur in Ausnahmefällen anzuwenden, da das radiogene Tochterprodukt ${ }^{40} \mathrm{Ca}$ nur in Mineralen, die sehr arm an gewöhnlichem Kalzium sind, mit ausreichender Genauigkeit nachweisbar ist. Das ${ }^{40} \mathrm{Ca}$ ist mit 96 g das weitaus hăufigste Isotop des gewöhnlichen Kalziums, das zudem noch selbst ein sehr häufiges Element darstellt. Einige Datierungen nach dieser Methode sind von HERZOG (1956) an Lepidolithen und Sylviniten durchgeführt worden.

Im Gegensatz hierzu ist die ${ }^{40} \mathrm{~A} /{ }^{40} \mathrm{~K}$-Methode wegen der fast totalen Abwesenheit von gewöhnlichem Argon (-Luftargon) weitaus hăufiger anwendbar. Obwohl wegen der für die Bestimmung des radiogenen ${ }^{4 O_{A}}$ sehr ungünstigen Häufigkeitsverteilung des gewöhnlichen Argons $\left({ }^{4 O_{A}}=99,7 \%\right)$ und der im Vergleich zum radiogenen ${ }^{40} \mathrm{Ca}$ zehnmal geringeren Menge schon kleine Mengen gewöhnlichen Argons die Meßgenauigkeit erheblich beeinträchtigen können, ist die Konzentration von (meist adsorbierten) gewöhnlichen Argons im allgemeinen so klein, daB die korrektur hierfür über das ${ }^{36} \mathrm{~A}$ genügend genau durchführbar ist. Erst bei extrem jungen oder kaliumarmen Material treten in dieser Hinsicht Schwierigkeiten auf. Die Argonbestimuing, die von GENTNER u. KLEY (1957), (1958) volumetrisch durchgeführt wurde, wird jetzt fast ausschlieBlich nach dem Verfahren der Isotopenverdünnungsanalyse vorgenommen, wobei ein sehr hoch in ${ }^{38} \mathrm{~A}$ angereichertes, sog. Spike-Argon, verwendet wird. Die meisten Anlagen basieren auf dem von WASSERBURG u. HAYDEN (1955) beschriebenen System, wobei dem beim Schmelzaufschluß der Probe frei werdenden Probenargon der ${ }^{38} \mathrm{~A}-\mathrm{Sp}$ ike zugegeben wird, und das in der anschließenden Reinigung aber CuO und Ti und Ausfrieren in Holzkohle rein gewonnene Argon im Massenspektrometer isotopisch analysiert wird. Anderungen und Verbesserungen wurden von WETHERILL et al. (1956), LONG u. KULP (1962) und anderen Bearbeitern eingefuhrt. Als Isotopen-Spike wird von CLUSIUS, Zürich, ein extrem reines $38_{\mathrm{A}}$ hergestellt

$$
\begin{array}{cccc}
38_{\mathrm{A}-\text { Spike Zürich; }} & 36{ }_{\mathrm{A}} & 38{ }_{\mathrm{A}} & 40{ }_{\mathrm{A}} \\
0.003 & 99.982 & 0.015 \\
\text { Atmosph. Argon; } & 0.337 \% & 0.063 \% & 99.6 \%
\end{array}
$$


Damit kann eine Meßgenauigkelt in der Argonbestimmung von besser als \pm 18 und im Modellalter von besser als $\pm 1.5 \%$ erreicht werden.

Als datierbare Minerale werden am häufigsten Biotite und Muskowite verwendet, weiterhin Feldspäte, das Gesamtgestein und auch kaliumarme Minerale, wie Albit (DAMON 1964), Kalzit und Flußspat (LIPPOLT u. GENTNER 1963) und Hornblende, wobei

kleinste Argonkonzentrationen von $10^{-7} \frac{\mathrm{cm}^{3}}{\mathrm{~g}}$ mit wenigen Prozent Fehlerbreite bestimnt werden. Für die Datierung von Sedimenten sind Glaukonite, deren Kaliungehalt in der gleichen Größenordnung wie für Biotite liegt, gut geeignet.

POLEVAYA et al. (1961) haben eine große Zahl von Glaukoniten aus verschiedenen Gebieten der U.S.S.R, und Chinas, die eine Altersspanne zwischen 40 ma bis $1200 \mathrm{ma}$ Jahren überdecken, untersucht und eine gute Ubereinstimmung mit den geologischen Daten erhalten, Ebenso hat eine vergleichsdatierung eines $10^{9} \mathrm{a}$ alten Glaukonits nach der $\mathrm{K} / \mathrm{Ar}$ - und der Rb/Sr-Methode von GULBRANDSEN et al. (1963) im Rahmen der Analysenfehler zu gleichen Ergebnissen gefuhrt.

Vulkanisches Glas und Tektite wurden von GENTNER u. LIPPOLT (1963) und von ZAHRINGER (1963) datiert, wobei mit Argongehalten von $5 \times 10^{-8} \mathrm{~cm}^{3} / \mathrm{g}$ Alter von $0.6 \mathrm{ma}$ mit einer Meßgenauigkeit von $\pm 10 \%$ ermittelt wurden. Extrem junge Alter von einigen $10^{5}$ a wurden von EVERNDEN et al. (1958) gemessen. SCHAFFER et al. (1961) konnten durch Vergleich mit Biotitdatierungen zeigen, daB vulkanisches Glas, sofern die Korngröße nicht zu gering ist, für die Datierung von vulkanischen Gesteinen geeignet ist. 


\subsection{Störungen des $\mathrm{K} / \mathrm{Ar}$-Verhältnisses}

Da das radiogene Tochterisotop einem gasförmigen Element angehört, ist ein Verlust des Tochterproduktes durch Diffusion aus dem Mineralverband oder eine zuwanderung radiogenen Argons, letzteres besonders bei $\mathrm{K}$-armen Mineralen, in noch stärkerem Maße zu befürchten als z.B. bej der Rb/Sr-Methode. DAMON (1964) fand in Albiten mit einem K-Gehalt von 0.008 : einen Uberschus von radiogenem Argon von $5 \times 10^{-7} \mathrm{~cm}^{3} / \mathrm{g}$, womit frühere Ergebnisse von DAMON u. KULP (1957) bestätigt werden. Im Gegensatz zu diesem sog. "ererbten" radiogenen Argon steht der Verlust aurch Diffusion, der bei Kalifeldspäten häufig beobachtet wird, wodurch diese Minerale leicht zu geringe scheinbare Alter ergeben.

Eine Untersuchung von ALDERICH u. WETHERILL (1958) an kogenetischen Glimmern und Feldspäten zeigt, daß etwa $90 \%$ der Vergleichsproben einen wert $\mathrm{R}=\left({ }^{40} \mathrm{Ar} /{ }^{40} \mathrm{~K}\right)$ Glimmer: ${ }^{40} \mathrm{Ar} /{ }^{40} \mathrm{~K}-$ Feldspat $>1$ ergeben, hingegen die $\mathrm{K}-\mathrm{A} x-\mathrm{Alter}$ der Glimmer mit den $\mathrm{Rb}-\mathrm{Sr}-\mathrm{Alt}$ ern eine befriedigende Ubereinstimung ergaben. GENTNER $u$. KLEY (1957) stellten fest, daß bei syngenetischen Glimmern und Feldspäten, letztere $5-78$, niedrigere $\mathrm{K} / \mathrm{Ar}$-Alter aufwiesen, was man durch Diffusionsverluste $z u$ erklären versuchte. Die Argon-Abgabe nimmt mit geringerer Korngröße zu, wie die Experimente von GENTNER u. KLAX (1957) gezeigt haben (Abb, 4. - 2). Die Argonabgabe mit zunehmender Mineralzerkleinerung geht nicht parallel mit der relativen VergröBerung des Oberflächen - zu Volumenverhältnisses, was darauf schließen läBt, daß das radiogene Argon im Feldspatkristall nicht gleichmäßig verteilt ist, sondern von den ehemaligen Kalium-Gitterplätzchen entfernt wird und sich an inneren Oberflächen konzentriert. Das Problem des Argonverlustes durch Diffusion ist von zahlreichen Autoren studiert worden. REYNOLDS (1957) hat die relative Argonabgabe

$f=\frac{C_{0}-C}{C_{0}} \quad(C=$ Argonkonzentration im Mineral $)$ nach 48-stündigem Verweilen auf einer vorgegebenen Temperatur $\left(300^{\circ}-900^{\circ}\right)$ durch Isotopenverdünnungsanalyse gemessen. Aus der Diffusions- 


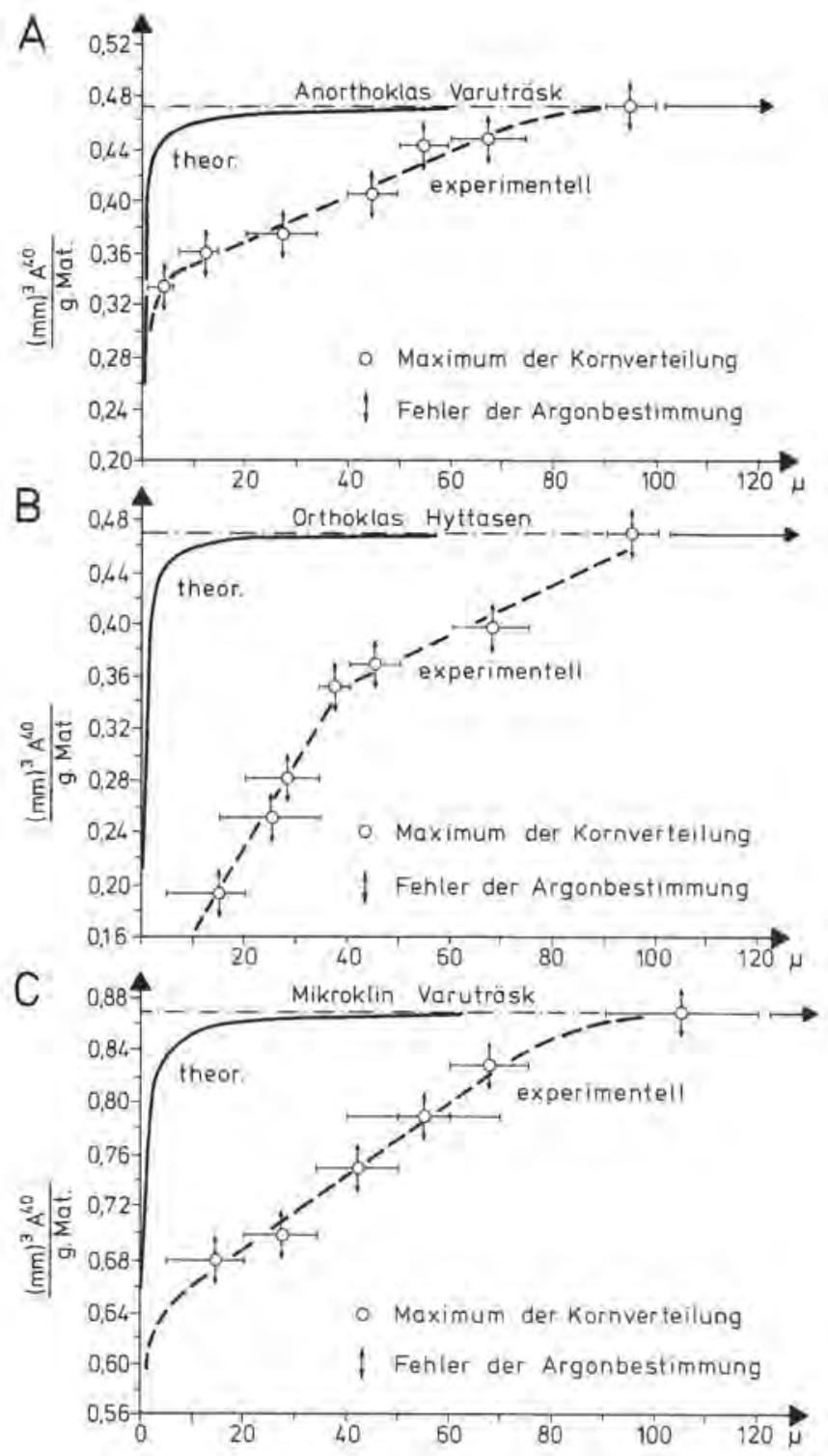

Abb. 4,-2: Argonverlust als Funktion der Korngröße (nach GENTNER und KLAY 1957) 
theorie erhält man für die Größe f die Bezeichnung

$$
\mathrm{f}=\frac{\mathrm{Co}_{0}-\mathrm{c}}{\mathrm{Co}}=1-\frac{6}{\pi^{2}} \sum_{1}^{\infty} \frac{1}{\mathrm{n}^{2}} \cdot \exp \left(\frac{n^{2} \pi^{2} \mathrm{D}, \mathrm{t}}{\mathrm{a}^{2}}\right) \quad(4 .-3)
$$

wobe 1

$$
\begin{aligned}
& \text { Co }=\text { A-Konzentration im Mineral vor der Erhitzung } \\
& \mathrm{C}=\text { " " nach " " } \\
& \text { " } \\
& \mathrm{a}=\text { Diffusionskonstante } \\
& t=\text { Diffusionszeit }
\end{aligned}
$$

bedeutet, (Vergl, auch JOST 1952). Für kleine f (f o.3) konvergiert diese Reihe sehr langsam, und die von CARLSLAW u. JAEGER (1959) angegebene Näherungsforme 1

$$
f=\frac{6}{\sqrt{\pi}} \sqrt{\frac{D \cdot t}{a^{2}}}-\frac{D t}{a^{2}}
$$

ist besser geeignet. Für eine homogene Argonverteilung sollte die Temperatur-Abhăngigkeit der Diffusionskonstante gegeben sein durch

$$
D=D_{0} \cdot \exp \left(-\frac{Q}{R T}\right)
$$

(mit $Q=$ Aktivierungsenergie $(\mathrm{cal} / \mathrm{mol}) ; \mathrm{R}=$ Gaskonstante; $\mathrm{T}=$ absol. Temperatur).

REYNOLDS (1957) erhält fux Feldspat Diffusionskonstanten von $>10^{-19} \mathrm{~cm}^{2} / \mathrm{s}$ bei etwa $30^{\circ} \mathrm{C}$, die von anderen Autoren jedoch nicht bestätigt werden. Mit einer derart hohen Diffusionskonstanten wären Diffusionsverluste von 20 - $30 \%$ bei hohen Altern selbst im Fall von großen Korngrößen möglich. Spätere Untersuchungen zeigen daher, daB die Diffusionskonstanten wesentlich kleiner sind.

AMIRKHANOFF et al. (1961) haben eine sehr umfangreiche untersuchung iber den Argonverlust durch Diffusion durchgeführt. Für den Gehalt von radiogenem Argon erhält man für homogenes kugelförmiges Material von Alter t: 


$$
4 O_{A}=\frac{\lambda_{K}}{\lambda_{B}+\lambda_{K}} \quad 4 O_{K} \frac{6}{\pi^{2}} \sum_{1}^{\infty} \frac{1}{n^{2}} \frac{\exp \frac{\left(\frac{-n^{2} \pi^{2} D t}{a^{2}}\right)}{1-\left(\frac{n \pi^{2}}{a}\right)} \frac{D}{\lambda} \exp (-\lambda t)}{(4,-6)}
$$

wobei ${ }^{40} \mathrm{~K}_{0}$ der ${ }^{40} \mathrm{~K}$-Gehalt zur zeit $t=0$ bedeutet mit $\mathrm{d}^{2}=\frac{\lambda \mathrm{a}^{2}}{\mathrm{D}}$ und $\mathrm{t}=\lambda t$ ergibt sich

$$
\frac{40_{A}}{4 O_{K}}=\frac{\lambda_{K}}{\lambda_{K}+\lambda_{B}} \cdot \frac{6}{\pi^{2}} \sum_{I}^{\infty} \frac{1}{n^{2}} \frac{\exp \left(-n^{2} \pi^{2} \frac{1}{d^{2}} \tau\right)-\exp (-\tau)}{1-\frac{n^{2} \pi^{2}}{d^{2}}}
$$

oder

$$
\frac{4 O_{A}}{4 O_{K}}=\frac{\lambda_{K}}{\lambda_{K}+\lambda_{B}} \cdot \frac{6}{\pi^{2}} \sum_{1}^{\infty} \frac{1}{n^{2}} \frac{\exp \left[\left(1-\frac{n^{2} \pi^{2}}{d^{2}}\right) \lambda\right]-1}{1-\frac{n^{2} \pi^{2}}{d^{2}}(4 .-8)}
$$

Das ${ }^{40} \mathrm{~A} /{ }^{40} \mathrm{~K}_{\mathrm{O}}$-Verhältnis als Funktion des Alters $t$ ist in Abb. $4,-3$ für verschiedene Werte $\frac{1}{d^{2}}$ dargestellt. Man erkennt daraus, daB für $\frac{D}{a^{2} \lambda}<10^{-4}$ der Diffusionsverlust praktisch vernachlässigbar wird. Für Laborexperimente ist $\lambda_{t} \ll 1$ und $\frac{\pi^{2} D}{\lambda_{a}^{2}} \ggg 1, d . h$. die Argonnachbildung durch Kaliumzerfall ist während der Versuchsdaver neben dem (bei erhöhter Temperatur) stattfindenden Argonverlust vernachlässigbar, und man er- 


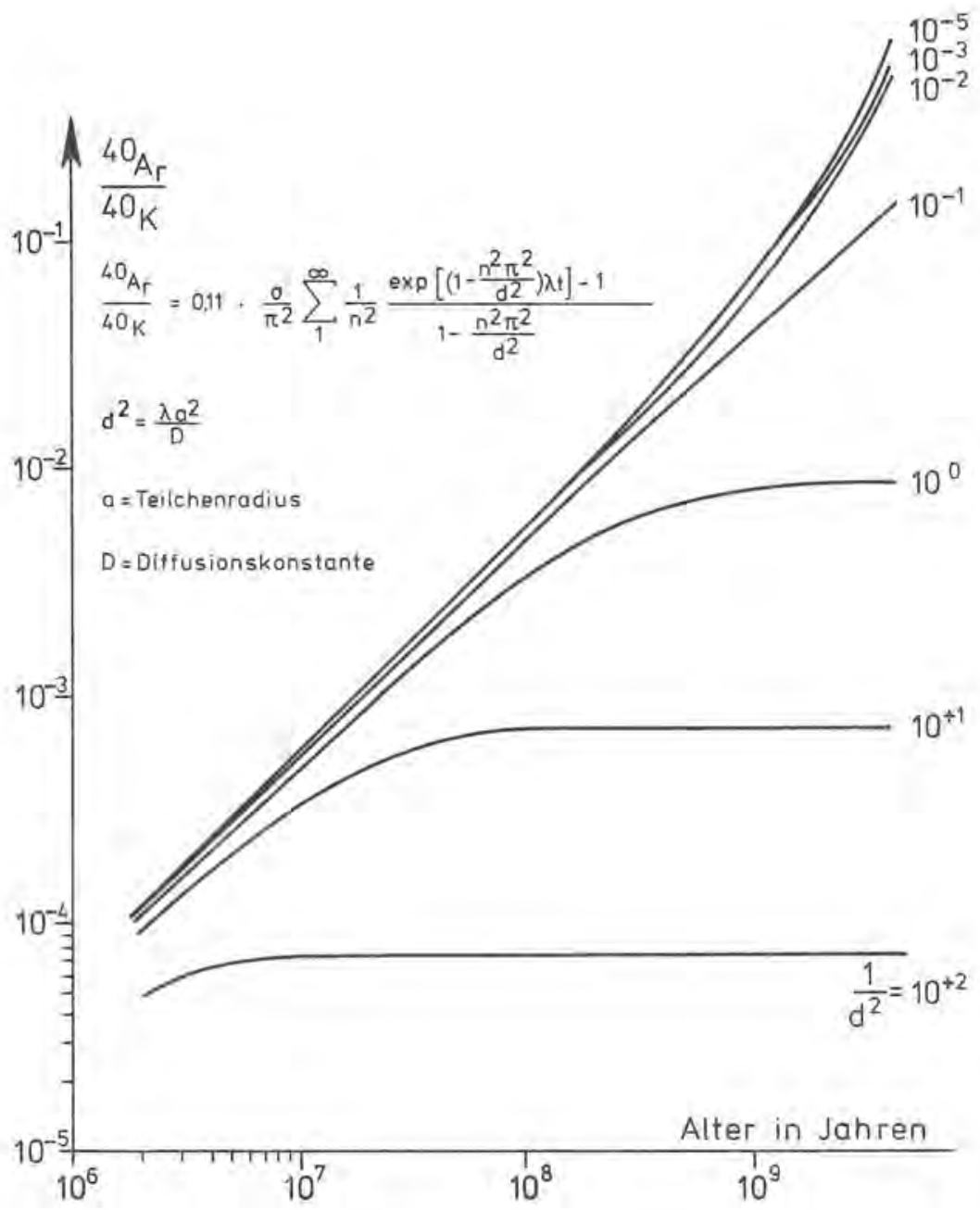

Abb. 4.-3: Das ${ }^{40} \mathrm{Ar} /{ }^{40} \mathrm{~K}$-Verhältnis als Funktion des Probenalters und der Diffusionskonstanten 
hä1t

$$
\frac{4 O_{A}(t)}{4 O_{A_{0}}}=\frac{6}{\pi^{2}} \sum_{1}^{\infty} \frac{1}{n^{2}} \exp \left(-\frac{n^{2} \pi^{2}}{a^{2}} D_{T} \cdot t\right), \quad(4 .-9)
$$

das Komplement zu der og. Formel für die relative Argonabgabe $f$. Hierbei ist $D_{T}$ die Diffusionskonstante bei der (erhöhten) absoluten Temperatur $T$ und $t$ die Ausheizzeit. Während REYNOLDS (1957) nur eine Messung bei $t=48 \mathrm{~h}$ durchfühte, haben AMIRKHANOFF et al. (1961) in kurzen zeitabständen innerhalb eines Zeitraumes von $44 \mathrm{~h}$ die abgegebene Argonmenge gemessen. Sie erhielten Diagramme ăhnlich dem in Abb. 4. - 2 gezeigten Beispiel für einen Phlogopit von $0.053 \mathrm{~mm}$ KorngröBe, Die Autoren kommen zu dem Schluß, daß drei verschiedene Bindungszustände des Argons existieren:

a) Im ersten zustand ist ein Teil des Argons (ca, 40 \&) relativ lose gebunden und entweicht bei Temperaturen unter $600^{\circ} \mathrm{C}$ schon nach einer halben Stunde Ausheizzeit. Danach tritt kein weiterer Argonverlust auf.

b) Das Argon im zweiten zustand verhält sich etwa in der Weise, wie es nach der Diffusionstheorie zu erwarten ist.

c) Ein geringer Teil des radiogenen Argons befindet sich in einem sehr fest gebundenen zustand und entweicht praktisch erst mit dem Schmelzen des Kristalls.

Das Argon im ersten Bindungszustand wird von AMIRKHANOFF et al. (1961) als adsorptiv gebunden angesehen, das der LANGMUIRGleichung gehorcht:

$$
\frac{\mathrm{V}}{\mathrm{Vm}}=\frac{\mathrm{b} \cdot \mathrm{p}}{\mathrm{I}+\mathrm{bp}}
$$

wobei $\frac{V}{V m}$ der relative Anteil des adsorbierten Gases und $p$ der Gasdruck ist. Für b gilt

$$
b=\frac{\exp (\stackrel{\mathrm{q}}{\mathrm{RT}})}{\mathrm{T}}
$$


Mit den Werten $\mathrm{q} / \mathrm{R} \approx 10^{3}$ und $\alpha \cdot \mathrm{p}=10 \mathrm{kann}$ dieser nur von der Temperatur und im Gegensatz zur Diffusion nicht von der Zeit abhängende Absorptionsanteil gut angenähert beschrieben werden. Aus den Diffusionskurven des zweiten Bildungszustandes ergeben sich für Feldspat Aktivierungsenergien von etwa $33-39$ Kcal/Mol und Diffusionskonstanten von $10^{-27}$ bis $10^{-32} \mathrm{~cm}^{2} / \mathrm{s}$ ungerechnet auf $300^{\circ} \mathrm{K}$.

Einen meBtechnisch anderen Weg zur Bestimmung der aus der Probe herausdiffundierten Argons haben FECHTIG et al. (1961) beschritten.

Durch Bestrahlung eines KCl-Einkristalles im Reaktor wurde im Kristall eine gleichmäBge Konzentration von radioaktivem ${ }^{39}$ A erzeugt durch die Reaktion

$$
{ }^{39} \mathrm{~K}(\mathrm{n}, \mathrm{p}){ }^{39} \mathrm{~A}
$$

bzw, bei Ca-haltigen Mineralen durch ${ }^{40} \mathrm{Ca}(n, \alpha){ }^{37} \mathrm{~A}$.

Das beim Ausheizen der Probe entweichende radioaktive Argon wird in einem direkt mit dem AusheizgefäB in Verbindung stehenden zählrohr gemessen. Auf diese Weise kann kontinuierlich während des Ausheizvorganges die Argonabgabe gemessen und die Diffusionskonstante bzw. der Wert $\mathrm{d}^{2}$ aus den MeBkurven nach der obengenannten Glelchung von CARLSLAW u. JAEGER (1959) berechnet werden. Fur KCl wurde eine Aktivierungsenergie von $47 \mathrm{Kcal} / \mathrm{Mol}$ erhalten. Fir Moldavit und Sanidin werden im $\frac{D}{a^{2}} / \frac{1}{T}$ Diagramm Gerade erhalten, während die Kurven von Phonolit und Augit Knicke aufweisen, die auf verschiedene Bindungsarten des radiogenen Argons hinweisen. Aus den MeBkurven werden Aktivierungsenergien für die verschiedenen Minerale von $30 \mathrm{bis} 90 \mathrm{Kcal} / \mathrm{Mol}$ und Diffusionskonstanten von $10^{-27} \mathrm{bis}$ $10^{-30}$ bei $\mathrm{z}$ immertemperatur errechnet.

Der Wert $\frac{D}{a^{2}}$ ist in allen Fallen kleiner als $10^{-4}$, so daB FECHTIG et al. (1961) übereinstimmend mit AMIRKHANOFF et al. (1961) zu dem Ergebnis kommen, daB bei Normaltemperatur ein Argonverlust durch Diffusion (vergl. Abb. 4. - 3) auch in geologischen zeiträumen ausgeschlossen werden kann. 


\subsection{Korrektur der Alterswerte bei Diffusionsverlust}

Eine Methode zur direkten Messung des $40_{A} /{ }^{40}$-Verhältnisses ohne Massenspektrometrie durch Neutronenaktivierung und Aktivitätsmessung ist von MERRIHUE und TURNER (1966) beschrieben und für die Datierung von Meteoriten angewendet worden. Durch Neutronenbestrahlung werden zwei Kernreaktionen erzeugt;

$$
{ }^{39} \mathrm{~K}(\mathrm{n}, \mathrm{p}){ }^{39} \mathrm{Ar} \rightarrow \mathrm{B}(0.6 \mathrm{MeV}, 270 \mathrm{a} \mathrm{HWZ})
$$

und

$$
{ }^{40} \mathrm{Ar}(\mathrm{n}, \mathrm{Y})^{41} \mathrm{Ar} \rightarrow \mathrm{B} \quad(1.2 \mathrm{MeV}, 2.5 \mathrm{MeV}, 1.87 \mathrm{~h} \mathrm{HWZ})
$$

d.h. , das entstandene ${ }^{39} \mathrm{Ar}$ ist proportional dem ${ }^{39} \mathrm{~K}$ und damit

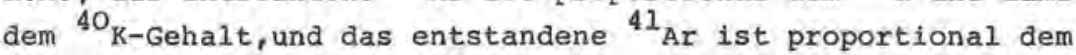
${ }^{40} \mathrm{Ar}-$ Gehalt der Probe. Beide hängen ferner von der Neutronendosis, den Wirkungsquerschnitten, usw. ab, so daB aus dem $A k-$ tivitätsverhältnis das ${ }^{40} \mathrm{Ar} /{ }^{40} \mathrm{~K}$-Verhältnis nur bei genauer Kenntnis all dieser GröBen bestimmbar ist. Bestrahlt man jedoch gleichzeitig unter gleichen Bedingungen, d.h., zusammen mit der zu datierenden Probe im Reaktor elne Probe bekannten Alters, so ist das Verhältnis der ${ }^{41} \mathrm{Ar} /{ }^{39} \mathrm{Ar}-$ Verhältnisse die-

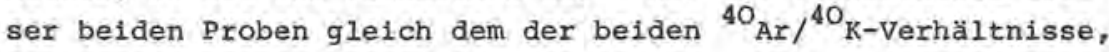
da alle die erwähnten unbekannten welteren Konstanten sich wegkürzen, also

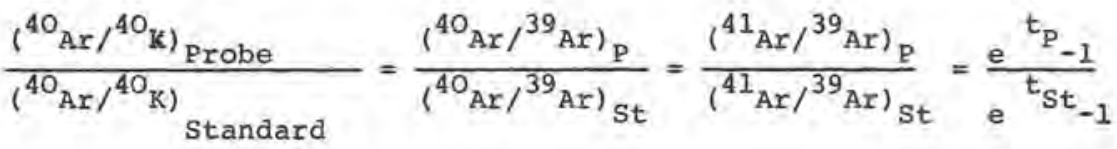

$$
(4 .-12)
$$

wobei die $\left({ }^{40} \mathrm{Ar} /{ }^{39} \mathrm{Ar}\right)$-Verhältnisse für die massenspektrome-

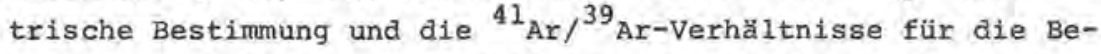
stimmung des Aktivitătsverhältnisses zu benutzen sind. Die radiometrische Methode setzt wegen der kurzen Halbwertszeit des ${ }^{41}$ Ar $(1.87 \mathrm{~h})$ eine sofortige Messung nach der Bestrahlung im Reaktor voraus, d.h., dieses Verfahren erfordert die Nähe eines Reaktors. Ein Nachteil dieses Verfahrens ist die Tat- 
sache, daB radiometrisch tiber das ${ }^{4 I_{A r}}$ nur die Gesamtmenge des ${ }^{40}$ Ar bestimmt wird, und eine unterscheidung zwischen radiogenem und atmosphärischem Argon ist nicht möglich ohne eine massenspektrometrische Bestimmung des ${ }^{40} \mathrm{Ar} /{ }^{36} \mathrm{Ar}$-Verhältnisses. Da aus diesem Grunde bereits eine Isotopenbestimmung mit dem Massenspektrometer erforderlich ist, - zumindest wenn nennenswerte Konzentrationen von atmosphärischem Argon zu erwarten sind, - dann kann auch das ${ }^{40} \mathrm{Ar} /{ }^{39} \mathrm{Ar}-\mathrm{Verhaltnis} \mathrm{massen-}$ spektrometrisch gemessen werden, wodurch die Probleme der radiometrischen Bestimmung des ${ }^{4 I}$ Ar mit seiner sehr kurzen Halbwertszeit entfallen. Dieses Verfahren hat den Vorteil gegenUber der separaten Bestimmung der ${ }^{40}$ Ar-Konzentration durch massenspektrometrische Isotopenverdünnungsanalyse einerseits und der Kaliumkonzentration an einer anderen Teilmenge der Probe,z.B. durch Flammenphotometrie, andererseits, daB hier belde Bestimungen glelchzeitig durchgeführt werden bzw. das Verhälnis dieser beiden Werte, das allein in die Altersbestimmungsformel eingeht, direkt auf dem Wege uber Isotopenverhältnisbestimmungen an derselben Teilmenge der Probe ermittelt wird, ohne die beiden Konzentrationen im einzelnen genau bestimmen zu mulssen.

Ein weiterer, sehr wesentlicher Vorteil dieser Methode besteht in der Möglichkeit, Ar-Verluste oder Gewinne durch Diffusion erkennen zu können.

Wird die Probe in der 1m Abschnitt 4.5 beschriebenen AufschluBapparatur langsam erhitzt, so wird zunächst das an den Kornoberflächen und störstellen in den Kristallen befindliche Argon entweichen. Bel früheren Argonverlusten im Laufe der geologischen Gesch1chte der Probe wird ebenfalls das Argon von diesen Partien der Kristalle am leichtesten entwichen sein. Das belm AusheizprozeB freiwerdende Argon, dessen zugehöriges ${ }^{40} \mathrm{Ar} /{ }^{40} \mathrm{~K}$-Verhältnis über das ${ }^{40} \mathrm{Ar} /{ }^{39} \mathrm{Ar}-$ Verhältnis gemessen wird, zeigt daher zunächst bei niedrigeren Ausheiztemperaturen im Falle elnes Argonverlustes der Probe geringere ${ }^{40} \mathrm{Ar} /{ }^{39} \mathrm{Ar}$-Verhältnisse und damit geringere scheinbare Alter. Mit Erhöhung der Aushelztemperatur wird auch Argon aus den inneren Teilen der Mineralkörner, das fester im Mineral gebunden ist, frei, 
und das ${ }^{40} \mathrm{Ar} /{ }^{39} \mathrm{Ar}-$ Verhältnis nimmt zu. Wird das gemessene ${ }^{40} \mathrm{Ar} /{ }^{39} \mathrm{Ar}-$ Verhältnis bzw. das daraus berechnete "Alter" gegen die relative Menge des freigewordenen Argons in einem Diagramm aufgetragen, so ist zunächst durch Erhöhung der Ausheiztemperatur mit zunehmender Argonmenge im Falle eines früheren Argonverlustes der Probe ein zunehmendes ${ }^{40} \mathrm{Ar} /{ }^{39} \mathrm{Ar}-$ Verhältnis zu erkennen, das mit weiter zunehmender freigesetzter Argonmenge einem konstanten Endwert zustrebt, der dem ursprïnglichen Alter der Probe entspricht. Aus dieser Kurve $1 \mathrm{a} B \mathrm{t}$ sich auch der relative Argonverlust berechnen. In günstigen Eällen läBt sich daraus auch das (jüngere) AIter des den Argonverlust verursacht habenden geologischen Ereignisses ermitteln. Mit diesem Verfahren hat TURNER (1970) das Alter von Mondproben bestimmt, die z.T. Argondiffusionsverluste bis zu 50 o erlitten haben. Die K/Ar-Datierung nach der konventionellen Methode hătte hier zu junge scheinbare Alter geliefert.

\subsection{Die Kallumanalyse}

Die klassischen, naBchemischen Methoden der Kaliumanalyse werden fur die $\mathrm{K} / \mathrm{Ar}-\mathrm{Alt}$ tersbestimmung praktisch nicht angewendet. Im allgemeinen stehen nur gexinge Probenmengen, besonders bei schwierig abzutrennenden Mineralen zur Verfügung, eine genügend genaue gravimetrische Kaliumbestimmung ist daher entweder nicht oder nur mit großem Aufwand möglich. Die Genauigkeit der K-Analyse sollte die der Ar-Analyse erreichen; denn beide gehen, zumindest bei Altern < 500 ma - linear in den Fehler des Alters ergebnisses ein.

Für Kaliumkonzentrationen $>0.2$ o wird die flammenphotometrisch Bestimmung mit internem Standard (Li) verwendet. Es werden dazu je nach Kalium-Konzentration ca. $100 \mathrm{mg}$ (Biotit, Muskowit, Glaukonit) bzw, 200 bis $300 \mathrm{mg}$ (Basalt, Hornblende) mit Flußsăure und Perchlorsăure aufgeschlossen und elne ebenfalls von der Kaliumkonzentration der Probe abhängige Teilmenge mit Pufferlösung und internem Standard versetzt und die Intensität der Kaliumlinie mit dem Emissions-Flammenphotometer gemessen

(SCHUHKNECHT u. SCHINKEL 1963). Im allgemelnen wird für die flammenphotometrische Methode eine untere Grenze von $\sim 0.2$ o 
Kalium in der Literatur genannt und für niedrigere Gehalte eine der nachfolgend beschriebenen Methoden empfohlen, es könmen jedoch bei ausgefeliten Analysenmethoden auch durchaus kleinere Kaliumkonzentrationen mit genügender Genaulgkeit bestimut werden. Durch automatisierte Analysenverfahren, bei denen mehrere Aufschlüsse derselben Probe abwechselnd mit Standardlösungen jeweils mehrfach gemessen werden, sind im Konzentrationsbereich zwischen 0,02 und 1 \% Kalium Reproduzierbarkeiten von 1 bis 2 i relativ und bel Konzentrationen oberhalb 5 o von \pm 0.4 o relativ erhalten worden. Damit kann diese äuBerst schnell und weitgehend automatisiert ablaufende AnaIysenmethode für die meisten geochronologischen Probleme angewendet werden.

Für sehr niedrige $\mathrm{K}$-Gehalte $(<0.1 \%)$ und kleine Probenmengen sind mit dex Isotopenverdünnungsanalyse oder der Neutronenaktivierungsanalyse Ergebnisse zu erzielen, die den Forderungen der Meßgenauigkeit genügen.

Das Prinzip der Isotopenverdunnungsanalyse ist bereits in Abschnitt 2. ausführlich beschrleben worden. Als "spike" dient ${ }^{41} \mathrm{~K}$ in mehr als $95 \%$-iger Anreicherung. Die untere Nachweisgrenze liegt bei $10^{-11} \mathrm{~g}$ Kalium, sie wird im wesentlichen durch Kontamination des Kaliums aus den verwendeten Chemikalien oder durch die Probenbearbeitung gegeben. Diese Methode der Kaliumbestimung ist u.a. erfolgreich bei steinmeteoriten angewendet worden (KIRSTEN et al, 1963).

Bei der Neutronenaktivierungsanalyse wird das bei der Reaktion

$$
{ }^{41} \mathrm{~K}(n, y){ }^{42} \mathrm{~K}
$$

entstehende Isotop ${ }^{42} \mathrm{~K}$ mit einer Halbwertszeit von $12,4 \mathrm{~h}$, 8-Energien von 3.58 und $1.99 \mathrm{MeV}$ und einer $\gamma$-Energie von $1.51 \mathrm{MeV}$ radioaktiv nachgewiesen, und die ${ }^{42} \mathrm{~K}$-Aktivität der Probe mit der einer am gleichen ort im Reaktor unter gleichen Bedingungen bestrahlten bekannten Kaliummenge verglichen. Zur Aktivitätsmessung ist das Kalium der Probe von den anderen (z,T. ebenfalls aktivierten) Elementen zu trennen. Hierzu wird die Probe vollständig in Lösung gebracht, eine bekann- 
te, im Vergleich zum in der Probe vorhandenen Kalium groBe Menge inaktiven Kaliums als Träger hinzugegeben und Kalium möglichst rein, aber nicht notwendigerweise quantitativ abgetrennt, und mit einem $B-Z a ̈ h l e r$ oder $Y$-Halbleiterdetektor mit Vielkanalanalysator gemessen. Durch Vergleich mit der Aktivität des Standards ergibt sich die Menge des zur Aktivitätsmessung gelangten Kaliums aus der bestrahlten Probe und durch Vergleich mit der bekannten zugegebenen Träger-KaliumMenge die chemische Ausbeute.

\subsection{Die Bestimmung des radiogenen Argons}

Die klassischen Methoden der Gasanalyse sind wegen der äuBerst kleinen Menge und der geforderten hohen relativen Genauigkeit nicht anwendbar. Ein Basalt mit 0.1 \% Kalium und einem Alter von $20 \times 10^{6}$ a enthält etwa $1 \times 10^{-7} \mathrm{~cm}^{3}$ (N.T.P.) radiogenes Argon. Adsorptiv kann eine nahezu gleich groBe Menge atmosphärischen Argons zusätzlich an das Gestein gebunden sein. Im Gegensatz zum radiogenen Argon, das ausschlieB1ich aus dem Isotop ${ }^{40} \mathrm{Ar}$ besteht, enthält das atmosphärische Argon $0.337 \%$ des Isotops ${ }^{36} \mathrm{Ar}$, das in einem Gemisch von radiogenem und atmosphärischem Argon als MaB für den Anteil des letzteren verwendet wird.

Zur Bestimmung der Menge und zur unterscheidung des radiogenen Argons von atmosphärischem Argon ist die massenspektrometrische Isotopenverdünnungsanalyse mit hochangereichertem ${ }^{38} \mathrm{Ar}$ als "spike" am besten geeignet. Die Probe wird in einer Hochvakuumanlage aufgeschlossen, d.h., entgast, was mit Hilfe von FluBmitteln, wie $\mathrm{NaOH}, \mathrm{Na}_{2} \mathrm{O}_{2}$ oder $\mathrm{Na}_{2} \mathrm{~B}_{4} \mathrm{O}_{7}$ bei Temperaturen um $1000^{\circ} \mathrm{C}$ oder durch Schmelzen der Probe ohne Flußmittel bei $1600^{\circ}$ bis $2000^{\circ} \mathrm{C}$ erfolgt. Die meisten Labors arbeiten nach der letztgenannten Methode, wobei die hohe Temperatur durch induktives Heizen eines Molybdän-Tiegels erreicht wird. Das der aufgeschmolzenen Probe entweichende Gas wird mit einer bekannten Menge des ogn. ${ }^{38}$ Ar-spikes versetzt und danach einem Reinigungsverfahren unterworfen. Das Gasgemisch wird über eine Kühlfalle zur Abtrennung des Wassers uber erhitztes Cuo zur Oxydation von 
$\mathrm{H}_{2} \mathrm{zu} \mathrm{H} \mathrm{H}_{2} \mathrm{O}$ und $\mathrm{CO} \mathrm{zu} \mathrm{CO}_{2}$ geleitet. Das Wasser und $\mathrm{CO}_{2}$ werden durch Kühlung mit flüssiger Luft ausgefroren. Titanschwamn wird als Getter für fast alle Nicht-Edelgase benutzt. Bei $850^{\circ} \mathrm{C}$ werden diese Gase nach einer Reaktionszeit von ca. Ih mit Ausnahme von Wasserstoff gebunden. Bei nachfolgender $\mathrm{Ab}-$ kuthlung auf $350^{\circ} \mathrm{C}$ bis $450^{\circ} \mathrm{C}$ reagiert dann auch der Wasserstoff. Das verbleibende Edelgasgemisch wird bei der Temperatur von flüssiger Luft an Holzkohle ausgefroren, wobei He nicht ausfriert, Das derart gereinigte Argon wird dann dem Massenspetrometer zur Isotopenanalyse zugefüht. Aus den gemessenen Isotopenverhältnissen $40_{\mathrm{Ar} /}^{38} \mathrm{Ar}$ und ${ }^{40} \mathrm{Ar} /{ }^{36} \mathrm{Ar}$ und der bekannten zugegebenen Menge ${ }^{38} \mathrm{Ar}$ wird die Menge des radiogenen ${ }^{40} \mathrm{Ar}$ und des atmosphärischen Argons berechnet. Alle die oben beschriebenen AufschluB- und Gasreinigungsvorgänge müssen in Ultrahochvakuum vorgenommen werden.

Eine eingehende Behandlung aller technischen Details ist z.B. bei ZÄHRINGER (1966) zu finden. 
LITERATUR

ALDRICH, L.T. \& WETHERILI, G.W.: Geochronology by radioactive Decay. - An. Rev. Nucl. Sci. 8, 257-298, 1958.

AMIRKHANOFF, K.I, BRANDT, S.B, \& BARTNITSKY, E.N. : Radiogenic argon in minerals and its migration. - Anal. N.Y. Ac. Sc. 91, Art. 2, 235-275, 1961.

CARLSLAW, H.S. \& JAEGER, J.C.: Conduction of heat in solids (2nd Ed) S. 92, Clarendon Press, Oxford 1959.

DAMON, P.E. \& KULP, J.L.: Argon in mica and the age of the Beryl Mt. N.H. Pegmatite. - Am. J. Sci. 255, $697-704,1957$.

DAMON, P.E.: Correlation and Chronology of ore deposits and Vulcanic rocks. - Univ. Arizona An. Progr. Rep. No. C 00-689-42-Contract AT (11-1)-689, 1964 .

EVERNDEN, J.F, , CURTIS, G.H. \& KISTLER, E.: Potassium Argon dating of Pleistocene Vulcanies. - "Quarternaria" 4, 13-17, 1958 .

FECHTIG, H., GENTNER, W. \& KALBITZER, S.: Argonbestiminung an Kaliumineralien IX. Messungen zu den verschiedenen Arten der Argondiffusion. - Geoch. Cosmochim. Acta 25, 297-311, 1961.

GULBRANDSEN, R.A., GOLDICH, S.S. \& THOMAS, H.H.: Glauconite from the Precambrian belt series, Montana. Science 140, 390-391, 1963.

GENTNER, W., PRÄAG, R. \& SMITS, F.: Argonbestimmung an Kaliummineralien II: Das Alter eines Kalilagers im unteren Oligozän. - Geoch. Cosmoch. Acta 4 , 11, 1953.

GENTNER, W. \& KLEY, W.: Argonbest1mmung an Kaliummineralien IV: Die Frage der Argonveriuste in Kalifeldspäten und Glimmermineralien. - Geochim. Cosmochim. Acta 12, 323-329, 1957.

GENTNER, W. KLEY, W.: Argonbestimmung in Kaliummineralien V: Altersbestimmung nach der $\mathrm{K} / \mathrm{A}$-Methode an Mineralien und Gesteinen des Schwarzwaldes. Geochim. Cosmochim. Acta 14, 98-104, 1958.

GENTNER, W. \& LIPPOLT, H.J.: Argonbestimung an Kaliumineralien XI: Geochim. Cosmochim. Acta 27, 191-200, 1963.

GLENDENIN, L.E.: Present Status of the Decay Constants. Ann. N,Y. Acad. Sci. 91, Art, 2, 166, 1961. 
HERZOG, L.F.: $\mathrm{Rb}-\mathrm{Sr}$ and $\mathrm{K}-\mathrm{Ca}$ Analyses and Ages Nucl. Processes in Geol. Settings. - Nucl. Sci. Ser. Rep. No. $19,1956$.

JOST, B.: Diffusion in Solids Liquids and Gases (Seite 45). Academic Press N.Y., 1952.

KIRSTEN, T, , KRANKOWSKY, D. \& ZAHRINGER, J.: Edelgas- und KaIiumBestimmungen an einer gröberen $\mathrm{Zahl}$ von Steinmeteoriten. - Geochim. Cosmochim. Acta 27, 13, 1963.

LIPPOLT, H.J. \& GENTNER, W.: K-A-Dating of some limestones and Fluorite, - "Radioactive Dating" IAEA, STI/PUB $68,239-244,1963$, IAEA, Wien.

LONG, I.E. \& KULP, J.L.: Isotopic Age Study of the Metamorphic History of the Manhattan and Reading Prongs. Geol. Soc. Am. Bull. 73, No. 8, 969-996, 1962.

MERRIHUE, C. \& TURNER, G.: Potassium Argon Dating by Activation with fast Neutrons. - Journ. Geoph. Res. 71, $2852-2857,1966$.

NIER, A.O.: A redetermination of the relative abundances of the isotopes of carbon, nitrogen, oxygen, argon and potassium. - Phys. Rev. 77, 789, 1950.

POLEVAYA, N.I, MURINA, G.A. \& KAZAKOV, G.A.: Utilisation of Glauconite in absolute Dating. - Anal. N.Y. Ac. Sci. 91, 298-310, 1961.

REYNOLDS, J,H.: Comparative Study of argon content and argon diffusion in mica and feldspar. - Geochim Cosmochim. Acta 12, 177-184, 1957.

SCHAEFFER, O.A., STOENNER, R.W, \& BASSETT, W.A.: Dating of tertiary vulcanic rocks by the potassium argon method. - Anal. N,Y, Ac. Sci. 91, Art. 2, $317-320,1961$.

SCHUHKNECHT, W. \& SCHINKEI, H.: Universalvorschrift zu Bestimmung von $\mathrm{K}, \mathrm{Na}, \mathrm{Li}$ von Proben jeder Zusammensetzung. - Z. Anal. Chem. 194, 161-183, 1963.

TURNER, G.: Argon-40/Argon-39 Dating of Lunar Rock Samples. Science $167,466-468,1970$.

WASSERBURG, G.J. \& HEYDEN, R.J.: ${ }^{40} \mathrm{~K} /{ }^{40} \mathrm{~A}$-Dating. - Geochim. Cosmochim. Acta $\underline{7}, 51,1955$.

WETHERILL, G.W., TILTON, G.R., DAVIS, G.L. \& ALDRICH, L.T.: New determinations of the age of the Bob Ingersoll pegmatite, Keystone, S. Dakota. Geochim. Cosmochim. Acta g, 292-297, 1956. 
ZAHRINGER, J.: Potassium Ages of the main tektive finds. IAEA Symp. Radioactive Dating, STI/PUB 68, 289-305, 1963, IAEA, Wien.

ZAHRINGER, J.: Potassium Argon Dating (compiled by 0.A. SCHAEFFNER and J,ZAHRINGER). - Springer Verlag, Berlin, Heidelberg, 1966. 


\section{DIE URAN-BLEI-METHODEN}

\subsection{Die direkten Uran-Blei-Methoden}

Das Uran war als natürliches radioaktives Isotop an fruhesten bekannt geworden, und man hat daher auch als erstes an uranpechblende Altersbestimungen nach der Formel

$$
\frac{\mathrm{Pb}}{\mathrm{U}}=\mathrm{e}^{\lambda t}-1
$$

durchgefuhrt, indem das Blei und das Uran quantitativ chemisch bestimut wurde. Da damals noch keine massenspektrometrischen Isotopenanalysen möglich waren, konnte man nicht zwischen gewöhnlichem, d.h, , bereits bei der Mineralbildung mit eingebautem und dem durch radioaktiven zerfall aus dem Uran entstandenen Blei unterscheiden, Damit war diese Methode auf reine Uranminerale, die möglichst frel von gewöhnlichem Blei sind, beschränkt, und die erhaltenen "Alter" waren lediglich als Maximalalter anzusehen.

Erst die Möglichkeit der Isotopenanalyse des Bleies schaffte die Voraussetzungen für die wirkliche Anwendung dieser Methode der Altersbestimung auf Minerale, die uran oder Thorium oder beides enthielten. Der entscheidende Durchbruch wurde jedoch erst durch die Isotopenvexdunnungsanalyse erreicht, denn damit war es möglich, auch Minerale, die nur geringe Konzentrationen der Mutterelemente uran und Thorium enthielten, die dafür aber nicht in speziellen Lagerstätten, sondern in vielen magmatischen Gesteinen vorkommen, wie z.B. Zirkon, Monazit, Titanit usw., zu datieren.

Die drei in der Natur vorkommencen langlebigen radioaktiven Isotope ${ }^{238} \mathrm{U},{ }^{235} \mathrm{U}$ und ${ }^{232} \mathrm{Th}$ zerfallen über mehrere ihrerseits wieder radioaktive $\mathrm{zwischenkerne} \mathrm{zu}$ den stabilen Isotopen ${ }^{206} \mathrm{~Pb},{ }^{207} \mathrm{~Pb}$ und ${ }^{208} \mathrm{~Pb}$. Nach einer Anlaufzeit, die groB im Vergleich zur längsten Halbwertszeit dieser radioaktiven Tochterisotope ist, wird für die gesamte Zerfallreihe (Abb.5.-1) 


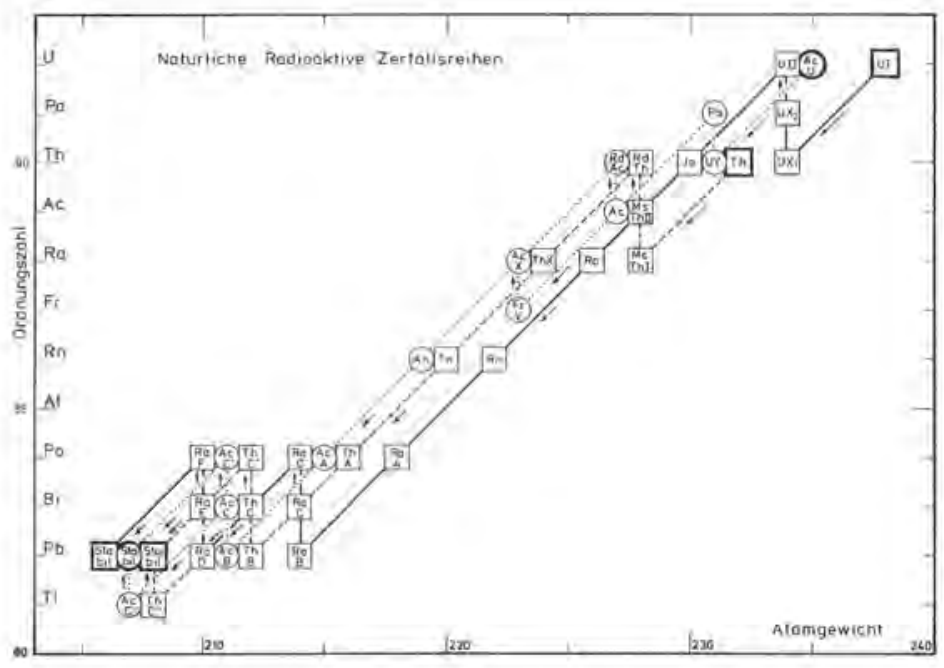

Abb. 5,-1: Die natürlichen radioaktiven Zerfallsreihen (ausführlich im Anhang)

der Zustand des radioaktiven Gleichgewichtes erreicht, und fur jedes zerfallende Uran- oder Thoriumatom entsteht ein entsprechendes Bleiisotop, d.h., für die Berechnung des. Anwachsens des radiogenen Bleies kann man so tun, als ob das Mutterisotop (z.B. ${ }^{238} \mathrm{U}$ ) direkt zum stabilen Tochterisotop (z.B. $\left.{ }^{206} \mathrm{pb}\right)$ zerfallen würde. Es kann daher die allgemeine Beziehung für das zeitliche Anwachsen des Tochter-MutterAtomzahlenverhältnis auch für diese drei Isotopenpaare angewendet werden, und es gelten folgende Formeln:

$$
\begin{aligned}
& \frac{{ }^{206} \mathrm{~Pb}^{+}}{238 \mathrm{U}}=e^{\lambda 238^{\mathrm{t}}}-1 \\
& \frac{{ }^{207} \mathrm{~Pb}^{+}}{235 \mathrm{U}}=e^{\lambda 235^{\mathrm{t}}}-1 \\
& \frac{{ }^{208} \mathrm{~Pb}^{+}}{232 \mathrm{Th}}=\mathrm{e}^{\lambda 232^{\mathrm{t}}}-1
\end{aligned}
$$


wobei $\mathrm{Pb}^{+}$das radiogene, d.h., durch den Zerfall der entsprechenden Mutterisotope entstandene Bleilsotop gemeint ist. Dieses rein radiogene Blei läBt sich rechnerisch von dem gewöhnlichen Blei, das die Isotope ${ }^{204} \mathrm{pb},{ }^{206} \mathrm{~Pb},{ }^{207} \mathrm{pb}$ und ${ }^{208} \mathrm{~Pb}$ enthält, mit Hilfe des nicht durch radioaktiven Zerfall entstehenden und nur im gewöhnlichen Blei enthaltenen Isotops ${ }^{204} \mathrm{~Pb}$ trennen, wenn die Isotopenverhaltnisse ${ }^{206} \mathrm{pb} /{ }^{204} \mathrm{~Pb},{ }^{207} \mathrm{~Pb} /{ }^{204} \mathrm{pb}$ und ${ }^{208} \mathrm{pb} /{ }^{204} \mathrm{pb}$ dieses gewöhnlichen Bleies bekannt sind. Diese Werte können im aligemeinen an mit den zu datierenden $U$ - bzw. Th-Mineralen genetisch korrelierbaren reinen $\mathrm{Pb}-\mathrm{Mineralen}$ bestimmt wexden. AuBerdem besteht noch die Möglichkeit, sie aus mehreren, gleichalten Proben mit Hilfe eines ${ }^{206} \mathrm{~Pb} /{ }^{204} \mathrm{~Pb}-{ }^{238} \mathrm{U} /{ }^{204} \mathrm{~Pb}$, bzw. ${ }^{207} \mathrm{~Pb} /{ }^{204} \mathrm{~Pb}-{ }^{235} \mathrm{U} /{ }^{204} \mathrm{~Pb}$ oder ${ }^{208} \mathrm{~Pb} /{ }^{204} \mathrm{~Pb}-{ }^{232} \mathrm{Th} /{ }^{204} \mathrm{~Pb} \mathrm{Dia}-$ gramms ganz analog zu dem NICOLAYSEN-Diagramm bei der Rb/SrMethode zu bestimuen.

Die in den Formeln $(5 .-2)$ benötigten Zerfallskonstanten sind in Tabelle $(5,-1)$ zusammengestellt.

Tabelle $5 .-1$

Zerfallskonstanten und Halbwertszeiten der Uranund Thorium-Isotope

\begin{tabular}{|ccc|}
\hline Isotop & $\begin{array}{c}\text { Zerfallskonstante } \\
\text { in } 10^{-10} \mathrm{a}^{-1}\end{array}$ & $\begin{array}{c}\text { Halbwertszeit } \\
\text { in } 10^{9} \mathrm{a}\end{array}$ \\
\hline $238_{\mathrm{U}}$ & 1,537 & $4,51 \pm 0,01$ \\
$235 \mathrm{U}$ & 9,722 & $0,713 \pm 0,016$ \\
$232_{\mathrm{Th}}$ & 0,488 & $14,2 \pm 0,2$ \\
\hline
\end{tabular}

Die Funktionen e $e^{t}-1$ sind ebenso wie die später erwähnte Funktion $\$ \operatorname{der}{ }^{207} \mathrm{~Pb} /{ }^{206} \mathrm{~Pb}$ Altersbestimung von STIEF \& STERN (1959) tabelliert, so daß dort für die gemessenen $\mathrm{Pb} / \mathrm{U}, \mathrm{Pb} / \mathrm{Th}$ bzw. ${ }^{207} \mathrm{~Pb} /{ }^{206} \mathrm{~Pb}$-Verhaltnisse das Alter $t$ direkt entnommen werden kann. In $\mathrm{Abb}, 5,-2$ sind sie zum besseren 


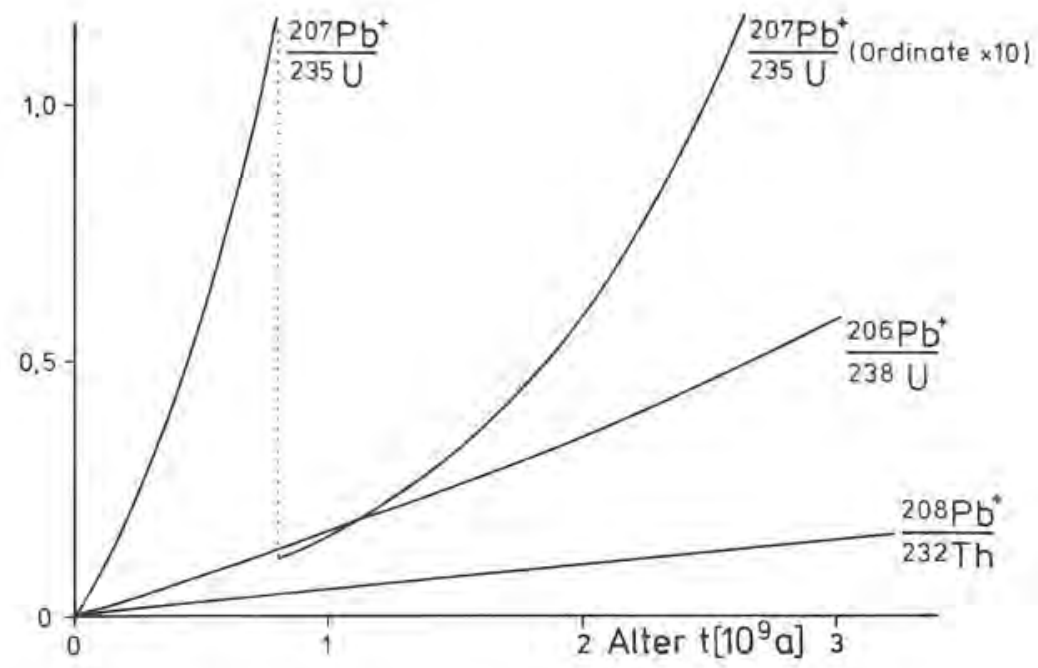

Abb. 5. -2: Die $\mathrm{Pb} / \mathrm{U}$ und $\mathrm{Pb} / \mathrm{Th}$ - Verhältnisse als Funktion des Alt,

Verstänanis noch einmal graphisch dargestellt. Es ist hier gut zu sehen, wie das ${ }^{207} \mathrm{~Pb} /{ }^{235} \mathrm{j}$-Verhältnis wegen der kleinen Halbwertszeit des ${ }^{235} \mathrm{U}$ am schnellsten zunimmt.

\subsection{Die abgeleitete Blei-Blei-Methode}

Durch Division der ersten und zweiten der Formeln 5,-2 erhält man eine weitere Formel, die, da das Verhältnis der Uranisotope ${ }^{235} \mathrm{U} / 238 \mathrm{U}=1 / 137$ eine universelle Konstante ist, nur noch das Verhältnis der radiogenen ${ }^{206} \mathrm{~Pb}$ und ${ }^{207} \mathrm{~Pb}$ Isotope enthălt.

$$
\frac{{ }^{207} \mathrm{~Pb}^{+}}{206 \mathrm{~Pb}^{+}}=\frac{1}{137} \cdot \frac{e^{\lambda_{235 t}-1}}{e^{\lambda 238^{t}}-1}
$$


Diese Formel zur sog. Blei-Blei-Altersbestimung von Uranmineralen hat u.a, den großen Vorteil gegenüber den $\mathrm{Pb} / \mathrm{U}-\mathrm{For}-$ meln (5. -2), daß als einzige Meßgröße nur das Verhältnis zweier Bleilsotope, das im Idealfall direkt an dem von dieser Probe abgetrennten Blei gemessen werden kann, in die Rechnung eingeht. Eine quantitative Bestimmung der Blei- oder Uran-

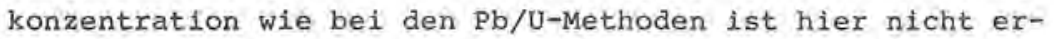
forderlich. Auch wird das Blei-Blel-Alter durch Blei- oder Uranverlust, wie später noch gezeigt wird, am wenigsten beeinflußt.

Der Nachteil der $\mathrm{Pb}-\mathrm{Pb}$-Methode liegt darin, daß bei kleinen Altern dieses auch bei hoher Präzision des MeBwertes ${ }^{207} \mathrm{~Pb}^{+}$, ${ }^{206} \mathrm{~Pb}^{+}$aus Formel $(5,-2)$ nur relativ ungenau ermittelt werden kann, wie sofort in der graphischen Darstellung (Abb.5.-3) der Formel $(5,-2)$ aus der geringen Steigung der Kurve bei kleinen Altern zu erkennen ist. Quantitativ ist das leicht aus einex Năherungsformel für Gl. (5.-3) zu ersehen. Ent-

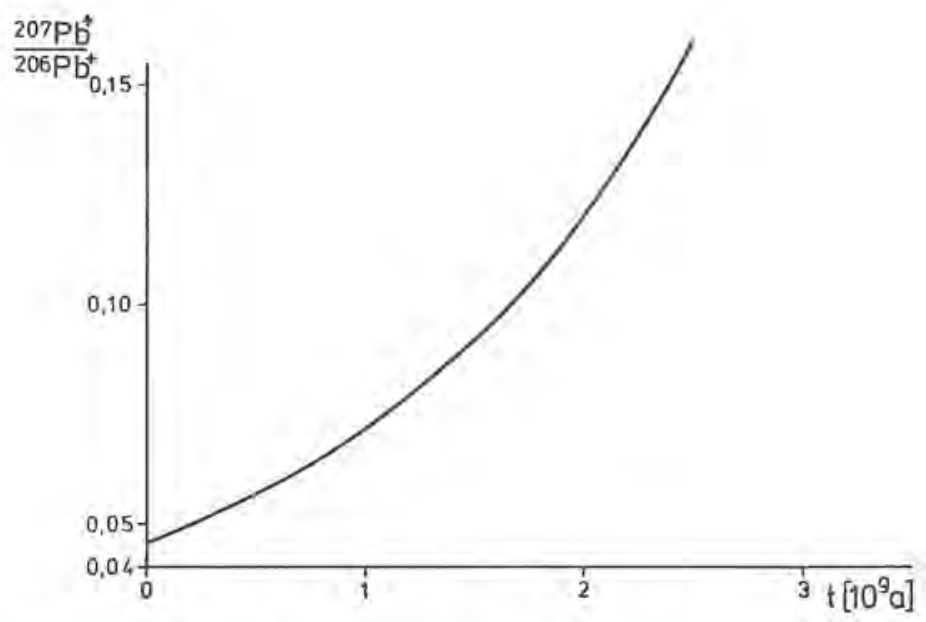

Abb. 5.-3: Das ${ }^{207} \mathrm{~Pb}^{+} /{ }^{206} \mathrm{~Pb}^{+}$-Verhältnis als Funktion des Alters 
wickelt man diese Gleichung für kleinere Alter $\left(\lambda_{\left.235^{t} \ll 1\right)}\right.$ in zähler und Nenner bis zum quadratischen Glied der Reihen für die Exponentialfunktion, so erhält man:

$$
\frac{207 \mathrm{~Pb}^{+}}{206 \mathrm{pb}^{+}}=\frac{1}{137.7} \cdot \frac{\lambda_{235}}{\lambda_{238}}\left(1+\frac{\lambda_{235}-\lambda_{238}}{2} \mathrm{t}\right) \quad(5 .-4)
$$

oder

$$
\frac{207 \mathrm{pb}^{+}}{206 \mathrm{pb}^{+}}=0,046 \cdot\left(1+4,09 \times 10^{-4} \mathrm{t}\right)
$$

mit $t$ in $10^{6} a$ für $t<2,5 \times 10^{8} a$.

Eine Ungenauigkeit in der ${ }^{207} \mathrm{pb}^{+} / 206 \mathrm{~Pb}^{+}$Isotopenbestimmung von \pm 18 ergibt also nach Formel $(5,-3)$ eine unsicherheit im Alter von $\pm 25 \mathrm{ma}$, die bei großen Altern z.B. bei $3 \times 10^{9} \mathrm{a}$ nur auf etwa $\pm 50 \mathrm{ma}$ ansteigt (Abb. $5,-4$ )

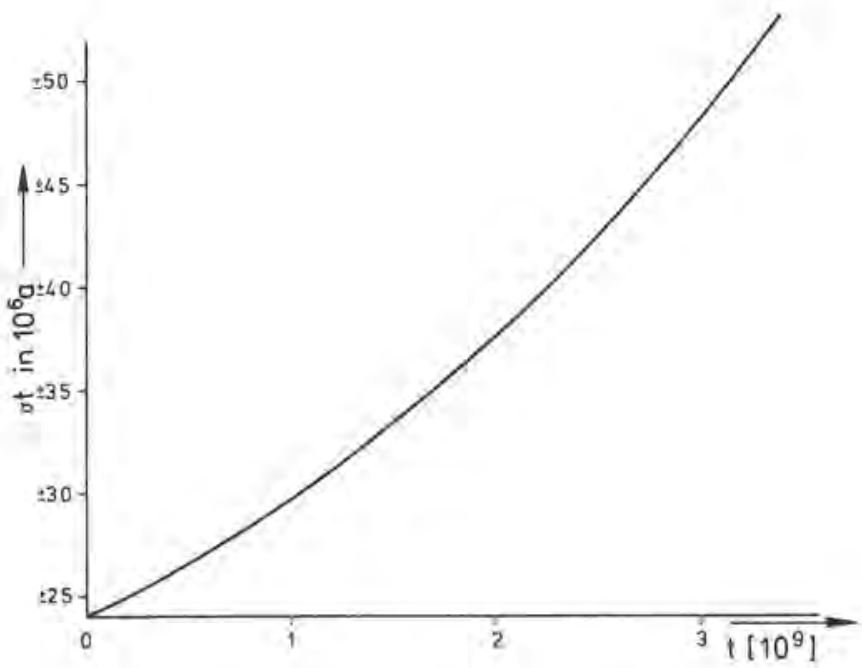

Abb, 5. -4: Fehler der ${ }^{207} \mathrm{pb}^{+} /{ }^{206} \mathrm{pb}^{+}$-Methode als Funktion des Alte 
Es ist damit verständlich, daB die ${ }^{207} \mathrm{~Pb}^{+} / 206 \mathrm{~Pb}^{+}$-Methode zur Bestimmung von kleinen Altern nicht geeignet ist, aber fur hohere Alter (etwa $>5 \times 10^{8}$ a) bereits eine sehr wertvolle Ergänzung $z u$ den beiden Uran-Blei-Methoden (Forme1 5,-2) darstellt. Die Unsicherheit in der Bestimung des Alters nach der ${ }^{207} \mathrm{~Pb} /{ }^{206} \mathrm{~Pb}$-Methode erhält bei kleinen Altern noch größere Fehler, wenn man berücksichtigt, daß man im allgemeinen auch in Uranineralien kein rein radiogenes Blei findet, sondern daneben noch gewöhnliches Blei, das bekannt11 ch ja auch die Isotope 206 und 207 enthält, vorhanden ist, das bereits bei der Mineralbildung mit eingebaut worden ist. Dieses mit dem Index Null in der folgenden Formel gekennzelchnete Ble1 1st von der Gesamtkonzentration ${ }^{206} \mathrm{~Pb}$ und ${ }^{207} \mathrm{~Pb}$ der Bleiisotope 206 und 207 abzuziehen. Formel $(5,-3)$ wäre aIso zu modifizieren zu

$$
\frac{{ }^{207} \mathrm{pb}^{+}}{206 \mathrm{~Pb}^{+}}=\frac{{ }^{207} \mathrm{pb}-{ }^{207} \mathrm{~Pb}_{\mathrm{o}}}{{ }^{206} \mathrm{~Pb}-{ }^{206} \mathrm{~Pb}}=\frac{1}{137} \frac{e^{\lambda 235^{t}}-1}{e^{\lambda 238^{t}}-1}
$$

teilt man $\mathrm{z}$ ahler und Nenner der linken Seite obiger Gleichung durch die zeitlich konstante Konzentration des nicht radiogenen Bleilsotops ${ }^{204} \mathrm{~Pb}$, so erhält man

$$
\frac{{ }^{207} \mathrm{~Pb}}{206 \mathrm{~Pb}} \cdot k=\frac{1}{137} \frac{\mathrm{e}^{\lambda_{235^{t}}}-1}{\mathrm{e}^{\lambda_{238^{t}}}-1}
$$

mit $k=\frac{1-\frac{\beta_{0}}{\beta_{0}}}{1-\frac{\alpha_{0}}{\alpha}}$

wobei $\alpha=\frac{{ }^{206} \mathrm{~Pb}}{{ }^{204} \mathrm{~Pb}} ; \quad \alpha_{0}=\frac{{ }^{206} \mathrm{~Pb}}{204{ }_{\mathrm{Pb}}} ; \quad B=\frac{{ }^{207} \mathrm{~Pb}}{204 \mathrm{~Pb}} ; \quad B_{O}=\frac{{ }^{207} \mathrm{~Pb}}{204{ }_{\mathrm{Pb}}}$ 
die auf das zeitlich konstante (d.h., ${ }^{204} \mathrm{~Pb}={ }^{204} \mathrm{~Pb}$ ) Bleiisotop 204 bezogenen Isotopenverhăltnisse des gesamten bei der Messung vorliegenden Bleies bzw. des gewöhnlichen Bleies (Index Null) sind.

Der sich aus den prozentualen Meßfehlern dieser Isotopenverhältnisse zusätzlich ergebende Fehler im korrigierten 207/ 206-Verhăltnis berechnet sich, wenn man das Verhältnis der radiogenen $\mathrm{Pb}$-Isotope mit $\phi$ bezeichnet, also

$$
\frac{207 \mathrm{~Pb}^{+}}{206 \mathrm{~Pb}^{+}}=\Phi, \mathrm{zu} \text { : }
$$

$$
\left(\frac{\sigma \Phi}{\phi}\right)^{2}=\left(\frac{\alpha_{0}}{\alpha-\alpha_{0}}\right)^{2}\left\{\left(\frac{\sigma \alpha}{\alpha}\right)^{2}+\left(\frac{\sigma \alpha}{\alpha_{0}}\right)^{2}\right\}+\left(\frac{\beta_{0}}{\beta-\beta_{0}}\right)^{2} \cdot\left\{\left(\frac{\sigma \beta}{\beta}\right)^{2}+\left(\frac{\sigma \beta_{0}}{\beta_{0}}\right)^{2}\right\}
$$

Dieser Fehler wird, wie sofort zu sehen ist, sehr groß, wenn neben dem gewöhnlichen Blel nur geringe Mengen radiogenen Bleies vorhanden sind, also $\alpha$ nur wenig größer als $\alpha_{0}$ und $\beta$ entsprechend wenig größer als $B_{0}$ ist. Bel relativ großen Konzentrationen von radiogenem Blei, also hohen Urankonzentrationen und großen Altern sowie bei kleinen Konzentrationen von gewöhnlichem Blei wird dieser Fehlerbeitrag aber klein,

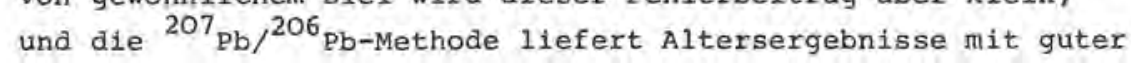
Genauigkeit.

\subsection{Diskordante Uran-Blei-Alter}

Falls die Voraussetzung erfüllt ist, daß für die Elemente Blei, Uran und Thorium sowie für alle Zwischenprodukte der Zerfallsreihen die zur Analyse gelangende Probe für die gesamte Dauer ihrer geologischen Geschichte ein geschlossenes system gewesen ist, d.h., daß keines dieser Elemente in dieser Zeit zu- oder abgeführt worden ist, so müssen die nach den Blei-Uran-Methoden (Formel 5,-2) und nach der BleiBlei-Methode (Foxme1 5,-5) erhaltenen Alterswerte im Rahmen 
ihrer MeBfehler gleich sein. Viele Uran-Blei-Datierungen haben auch solche "konkordanten" Altersergebnisse ergeben, aber das ist keineswegs immer der Fall. Sehr häufig wurden auch ModellAlter Folgen ermittelt, derart daß

$$
t(208 / 232)<t(206 / 238)<t(207 / 235)<t(207 / 206)<t,
$$

d.h., die nach den verschiedenen Methoden erhaltenen "Alter" sind in der og. Folge alle kleiner als das wirkliche Kristallisationsalter. Seltener findet man die ungekehrte Folge. Es läßt sich nun zeigen, daß sich bei einem späteren Bleirerlust des Uranminerals, z.B. đurch den Einflus einer Metamorphose oder durch Verwitterungsprozesse, die og. Folge dex scheinbaren "Alter" ergibt. Ganz offensichtlich ist also die bisher vorausgesetzte Annahme, die Mineralprobe als geschlossenes System ansehen zu können, sehr hăufig nicht erfüllt, und ein wirkliches Alter kann aus den Analysenergebnissen mit den einfachen Formeln $(5,-2)$ nicht mehr errechnet werden. Durch den glücklichen Umstand, daß bei den beiden uran-Blei"Uhren" sowohl das radioaktive Mutterelement, nämlich Uran, als auch das radiogene stabile Endprodukt, nämlich Ble1, für beide Zerfallsrethen chemisch glelch sind, ist es möglich, auch noch eine "Zwei-Ereignis-Geschichte" mit dem primären Bildungsalter und dem sekundären Metamorphosealter datieren zu können. Die mathematische Behandlung dieses Problems und die Ableitung der für einen solchen Fall gultigen Gleichungen, die anstelle der Gleichungen $(5,-2)$ anzuwenden sind, ist sehx einfach und soll daher hier kurz dargestellt werden.

Ein Uranmineral habe sich vor $t_{0}$ Jahren gebildet, und vor $t_{I}$ Jahren sei ein Teil des bis dahin gebildeten radiogenen Bleies $\mathrm{Pb}^{+}\left(t_{1}\right)$ durch einen der oben erwähnten prozesse aus dem Mineral entfernt worden, so daB nur noch $x .{ }^{+} \mathrm{Pb}\left(t_{1}\right)$ im Mineral verblieben ist. Die uranmenge $U$ läßt sich dann rechnerisch in zwei Komponenten aufspalten, und zwar die Menge $x$. $U$, die zum Zeitpunkt $t_{1}$ kein Blei, und die Menge $(1-x) v$, die zur Zeit $t_{1}$ alles bis dahin gebildete Blel verloren hat. Für den ersten Teil hat die Bildung des heute vorhandenen 
radiogenen Bleies vor $t_{0}$ Jahren, für den zweiten Teil erst vor $t_{1}$ Jahren begonnen. Es sind also für die diesen beiden Uran-Teilmengen entsprechenden Bleimengen $\mathrm{Pb}_{1}$ und $\mathrm{Pb}_{2}$ die Gleichungen $(5,-2)$ getrennt für die Alter $t_{0}$ und $t_{1}$ einzusetzen

$$
\mathrm{Pb}_{1}=x u\left(e^{\lambda t_{0}}-1\right) ; \quad \mathrm{Pb}_{2}=(1-x) U\left(e^{\lambda t_{1}}-1\right) .
$$

Die Sume dieser beiden Bleikomponenten liefert nach Division durch U für die beiden $\mathrm{Pb} / \mathrm{U}$-Verhältnisse

$$
\begin{aligned}
& \frac{{ }^{206} \mathrm{~Pb}^{+}}{{ }^{238} \mathrm{U}}=x\left(\mathrm{e}^{\lambda_{238^{\mathrm{t}}}{ }}-1\right)+(1-x)\left(\mathrm{e}^{\left.\lambda_{238^{\mathrm{t}} 1_{-1}}\right)}\right. \\
& \frac{{ }^{207} \mathrm{~Pb}^{+}}{235_{\mathrm{U}}}=x\left(\mathrm{e}^{\lambda_{235^{\mathrm{t}} \mathrm{O}}}-1\right)+(1-x)\left(\mathrm{e}^{\lambda_{235^{\mathrm{t}}}}-1\right)
\end{aligned}
$$

Diese Gleichungen sind für ein "Zwei-Ereignis-Modell" anstelle von Gl. $(5,-2)$, die fur das einfache "Ein-EreignisMocel1" gelten, zu setzen. Sie vereinfachen sich für die beiden Spezialfälle $x=1, d . h .$, kein Bleiverlust, und $x=0$, d.h., vollständiger Bleiverlust, bei $t=t_{1} z u$ Gl. $(5 .-2)$ und ergeben, da im ersten Fall die störung zur zeit $t_{1}$ nicht vorhanden ist, das primäre Alter $t_{0}$ und im zweiten Fall, da durch den vollständigen Bleiverlust bei $t_{1}$ ein neuer Anfangspunkt gesetzt wird, nur das jüngere Alter $t_{1}$. Wahrend bei dem einfachen Modell eine Probe eines Uraninerals durch die Anwendung der beiden Uran-Blei-Methoden zwei Gleichungen mit nur einer Unbekannten, nämlich dem Alter $t$, also ein uberbestimntes Gleichungssystem lieferte, das damit schon durch Ubereinstimmung bzw. Nichtübereinstimmung der Ergebnisse eine Kontrolle dieses Modelis erlaubte, sind in den Gleichungen $(5,-7)$ des erweiterten Modells, in dem jeder probe zugehörigen Gleichungspaar drei Unbekannte, nämlich $t_{0}, t_{1}$ und $x$ enthalten. Die Analyse einer Probe genügt also nicht zur Ermittlung dieser drei Werte. Hat man jedoch zwel proben mit der gleichen 
geologischen Geschichte, also primäre Bildung zur Zeit to' sekundäre Beeinflussung zur zeit $t_{1}$, aber verschieden starken Bleiverlust $\chi$, so stehen vier Gleichungen mit vier Unbekannten ( $t_{0}, t_{1}, x_{1}, x_{2}$ ) zur verfügung, die eine Lösung ermöglichen. Man kann also mit mindestens zwei proben aus einer geologischen Einheit auch noch ein komplizierteres geologisches Geschehen datieren. Jede weitere Probe erhöht die $\mathrm{Zahl}$ der Gleichungen um zwei und die Zahl der Unbekannten um nur eine, d.h., mit mehreren proben kann geprüft werden, ob ein "Zwei-Ereignis-Modell" vorliegt.

Diese etwas mathematische Betrachtung des durch die G1. (5.-7) beschriebenen Problems läßt sich sehr gut graphisch veranschaulichen:

Trägt man die Werte

$$
\mathrm{x}=\frac{207 \mathrm{~Pb}^{+}}{235 \mathrm{U}}=e^{\lambda_{235^{t}}-1}
$$

und

$$
y=\frac{206 \mathrm{~Pb}^{+}}{238 \mathrm{U}}=e^{\lambda_{238^{t}}}-1
$$

in einem $x-y$-Koordinatensystem als Funktion von $t$ gegeneinander auf, so erhält man die sog. "Concordia"-Kurve (Abb.5.-5). Die Quotienten der radiogenen Blei- und der entsprechenden Uranisotope

$\frac{207 \mathrm{~Pb}^{+}}{235 \mathrm{U}}$ und $\frac{206 \mathrm{~Pb}^{+}}{238 \mathrm{U}}$ aller Proben, die "konkordante" Alter, also nach der 207/235 gleiche Alter, wie nach der 206/238Methode liefern GI. (5.-2), liegen in diesem Diagramm auf der "Concordia". Ist ein Bleiverlust zu irgendelner späteren Zeit nach der Bildung aufgetreten, so liegt der ProbenmeBpunkt nicht mehr auf dieser Kurve, und die Auswertung der $\mathrm{Pb} / \mathrm{U}$-Verhältnisse nach Gl. $(5,-2)$ liefert verschiedene "diskordante" Alter für die beiden $\mathrm{Pb} / \mathrm{U}-M e t h o d e n$. Aus den für eine "Zwei-Ereignis-Geschichte" gültigen Gleichungen (5.-7) läBt sich nun folgendes ablesen: die $x$ - und $y$-Werte aller 


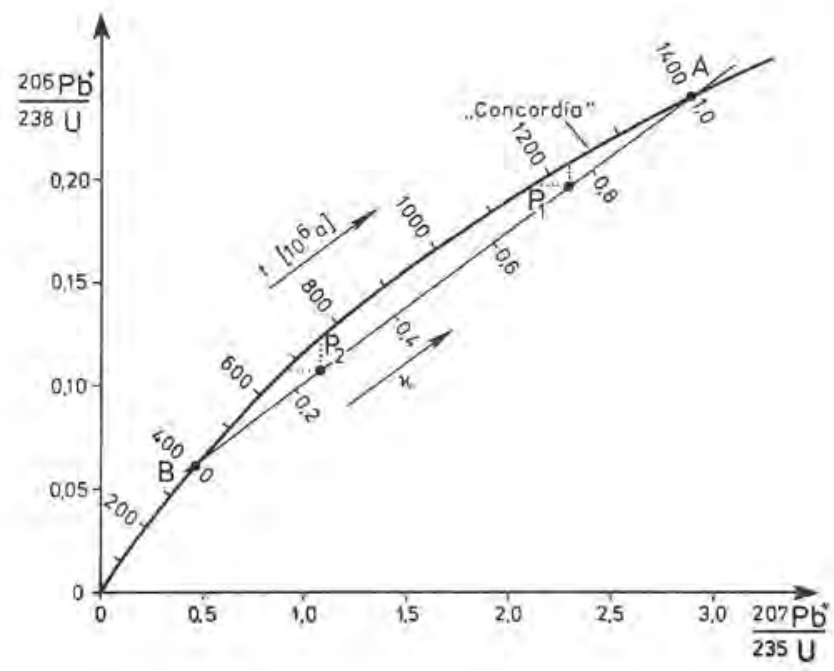

$\underline{\mathrm{Abb} .}$ 5.-5: Das ${ }^{207} \mathrm{~Pb}^{+} / 235 \mathrm{U}-{ }^{206} \mathrm{~Pb}^{+} /{ }^{238} \mathrm{U}$ - Concordia-Diagramm

Proben, die sowohl ein gleiches primäralter $t_{0}$ als auch ein gleiches Sekundäralter $t_{1}$ haben, sind rein lineare Funktionen von $x$, sie liegen also in dem "Concordia"-Diagramm auf einer Geraden. Für die beiden spezialfälle $x=1$ bzw. $x=0$ degenerieren die beiden Gleichungen $(5 .-7) \mathrm{zu}(5 .-2)$ und liefern konkordante Alter $t_{0} b z w, t_{1}, d . h .$, diese beiden Fälle liegen auch auf der "Concordia" und zwar an den $t_{0} b z w$. $t_{1}$ entsprechenden Punkten. Man kann also sagen:

Die $\mathrm{Pb} / \mathrm{U}-$ Verhältnisse aller Proben, die gleiches Primäralter $t_{0}$ und gleiches sekundäralter $t_{1}$, aber verschieden starke Bleiverluste haben, liegen im "Concordia"-Diagramm auf einer Geraden, (die manchmal auch als "Diskordia" bezeichnet wird), die die "Concordia" in den $t_{0}$ und $t_{1}$ entsprechenden Punkten schneidet. 
Dieses Ergebnis sol1 an einem in Abb. 5, -5 dargesteliten konkreten Beispiel nochmals veranschaulicht werden.

Ein 1400 ma altes Uramineral würde, wenn es bis heute keinen Uran- oder Bleiverlust erlitten hätte, die Werte

$\frac{206 \mathrm{~Pb}^{+}}{238 \mathrm{U}}=0,2400$ und $\frac{207 \mathrm{~Pb}^{+}}{235 \mathrm{U}}=2,900$ und $\frac{{ }^{207} \mathrm{~Pb}^{+}}{206 \mathrm{~Pb}^{+}}=0,0877$

haben (Punkt $A$ in Abb, 5.-5). Alle drei werte liefern nach Formel 5, -2 bzw. 5,-3 dasselbe Alter von $1400 \mathrm{ma}$.

Hat das Mineral jedoch vor 400 ma 25 \% seines Bleies verloren $(x=0,75)$, so wären die heutigen Blei-uran-Verhältnisse (Punkt $\mathrm{P}_{1}$ in $\mathrm{Abb}, 5 .-5$ )

$\frac{206 \mathrm{pb}^{+}}{238 \mathrm{U}}=0,1958 ; \quad \frac{207 \mathrm{pb}^{+}}{235 \mathrm{U}}=2,294 ;$ und $\frac{207 \mathrm{pb}^{+}}{206 \mathrm{pb}^{+}}=0,0850$

daraus wiurde man die scheinbaren "Alter"

$t(206 / 238)=1164 \mathrm{ma} ; t(207 / 235)=1226 \mathrm{ma} ; t(207 / 206)=1340 \mathrm{ma}$

erhalten. Bei einem Bleiverlust von 75 vor 400 ma $(x=0,25)$

erhalt man (Punkt $\mathrm{P}_{2}$ in $\mathrm{Abb}, 5,-5$ )

$\frac{206 \mathrm{~Pb}^{+}}{238 \mathrm{U}}=0,1075 ; \quad \frac{207 \mathrm{~Pb}^{+}}{235 \mathrm{U}}=1,0812 ;$ und $\frac{207 \mathrm{~Pb}^{+}}{206 \mathrm{~Pb}^{+}}=0,073$

mit dem scheinbaren "Alter"

$t(206 / 238)=664 \mathrm{ma} ; t(207 / 235)=754 \mathrm{ma} ; t(207 / 206)=1033 \mathrm{ma}$.

Falls es sich um einen reinen Bleiverlust zum Zeltpunkt $t_{1}$ handelt, und nicht um eine Zuführung von Uran, - beide Fälle sind hinsichtlich der Blei-Uran-Methoden nicht voneinander zu unterscheiden, - so ist eine dritte Gleichung dem System 5.-7 für das Verhältnis des radiogenen ${ }^{208} \mathrm{~Pb}$ und des ${ }^{232}$ Th hinzuzurügen, und es gilt entsprechend 


$$
\frac{20 \mathrm{~Pb}^{+}}{232 \mathrm{Th}}=x\left(\mathrm { e } ^ { \lambda _ { 2 3 2 ^ { \mathrm { t } } \mathrm { o } _ { - 1 } } ) + ( 1 - x ) } \left(\mathrm{e}^{\left.\lambda_{232^{\mathrm{t}}}{ }_{-1}\right)}\right.\right.
$$

und die gemessenen $\frac{208 \mathrm{~Pb}^{+}}{232 \mathrm{Th}}$ Verhältnisse müssen mit den aus dem $\mathrm{Pb} / \mathrm{U}$-Concordia-Diagramm ermittelten $t_{0}, t_{1}$ und $x$-Werten die obige Gleichung erfullen. Für unser hier diskutiertes Beispiel heibt das:

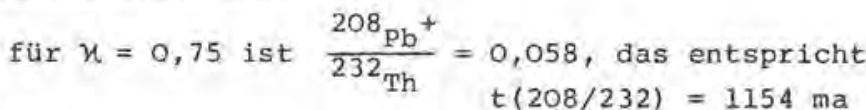

$$
\begin{aligned}
& \text { u. für } x=0,25 \text { ist }=0,0325 \text {, das entspricht } \\
& t(208 / 232)=654 \mathrm{ma}
\end{aligned}
$$

Das $\mathrm{Pb} / \mathrm{Th}$-Verhältnis erlaubt also in besonderen Fällen auch bei diskordanten Altern eine Kontrolle der aus den $\mathrm{Pb} / \mathrm{U}-$ Methoden ermittelten Primär- und Sekundäralter. Leider sind diese besonderen Fälle recht selten, denn die Voraussetzungen sind nur bei homogenen U-Th-Mischkristallen und reinem Bleiverlust gegeben. Sitzt hingegen das Th und damit auch das daraus entstandene ${ }^{208} \mathrm{~Pb}$ an einer anderen stelle im Mineral als das uran und damit das radiogene ${ }^{206} \mathrm{~Pb}$ und ${ }^{207} \mathrm{~Pb}$, so kann der durch Sekundärprozesse verursachte Bleiverlust für das ${ }^{208} \mathrm{~Pb}$ anders sein als für das ${ }^{206} \mathrm{~Pb}$ und ${ }^{207} \mathrm{~Pb}$.

Zirkon, wohl eines der bisher am häufigsten für die Datierung von Intrusivgesteinen nach den $\mathrm{Pb} / \mathrm{U}$-Methoden verwendete Mineral, ergibt sehr oft diskordante Alter, die durch eine solche $\mathrm{z}$ wei-Ereignis-Geschichte bedingt sind. Man beobachtet hier die für sekunaären Bleiverlust charakteristische Reihenfolge der Modellalter

$t_{1}<t(208 / 232)<t(206 / 238)<t 207 / 235<t 207 / 206<t_{0}$

Sind mehrere Proben solcher diskordanten $\mathrm{zirkone}$ aus einem Gestein vorhanden, so liegen sie jedoch in sehr vielen der bisher untersuchten Fälle im Concordia-Diagramm auf einer Geraden und aus deren Schnittpunkten mit der Concordia können Werte $t_{0}$ für Primär- und $t_{1}$ für das Sekundäralter entnommen werden. 
Schon Zirkonkonzentrate unterschiedlicher Korngröße derselben Gesteinsprobe können unterschiedliche Blelverluste und damit verschiedene diskordante Alter ergeben.

SILVER \& DEUTSCH (1963) haben aus einem Granodiorit Zirkone verschiedener Korngröße abgetrennt, deren scheinbare Alter, wie sie sich aus den $\frac{206 \mathrm{pb}^{+}}{238 \mathrm{U}}, \frac{207 \mathrm{~Pb}^{+}}{235 \mathrm{U}}$ und $\frac{{ }^{207} \mathrm{~Pb}^{+}}{206 \mathrm{pb}}+-$

Verhältnissen nach Gl, $(5,-2)$ errechnen in Tabelle 5.-2 zusammengestellt sind.

Tabelle 5.-2

Scheinbare Alter verschiedener Siebfraktionen von Zirkon aus einer Granodioritprobe nach SILVER \& DEUTSCH 1963.

\begin{tabular}{|l|r|r|r|}
\hline \multicolumn{4}{|c}{ Scheinbares Alter in ma } \\
\hline Siebfraktion & $t 206 / 238$ & $t 207 / 235$ & $t 207 / 206$ \\
R 200 mesh & $1070 \pm 20$ & $1270 \pm 20$ & $1630 \pm 20$ \\
R 300 mesh & $1030 \pm 20$ & $1230 \pm 20$ & $1610 \pm 20$ \\
R 400 mesh & $980 \pm 20$ & $1200 \pm 20$ & $1620 \pm 20$ \\
400 mesh & $995 \pm 20$ & $1205 \pm 20$ & $1610 \pm 20$ \\
UT R 300 & $227 \pm 30$ & $348 \pm 40$ & $1267 \pm 40$ \\
\hline
\end{tabular}

Im Concordia-Diagramm liegen diese vier Proben zusammen mit einem Uranothorit-Konzentrat aus dem gleichen Gesteinsstück auf einer Geraden (Abb, 5. -6), deren Schnittpunkte mit der "Concordia" ein Primäralter von $t_{0}=1660$ ma und ein Sekundäralter von $t_{1}=90 \mathrm{ma}$ ergeben. Die Autoren konnten auch zeigen, daß bei zonarem Aufbau der Zirkone durch schichtweises Ablösen einzelner Fraktionen unterschiedliche diskordante Alter erhalten werden, die aber alle auf derselben "Diskordia" liegen.

Neben diesem "Zwei-Ereignis-Modell", das in vielen Fällen mit diskordanten Altern erfolgreich angewendet worden ist, kann auch noch ein sog. "Diffusionsmodell" der tatsächlichen geologischen Geschichte besser angepaßt sein. Das oben aus- 


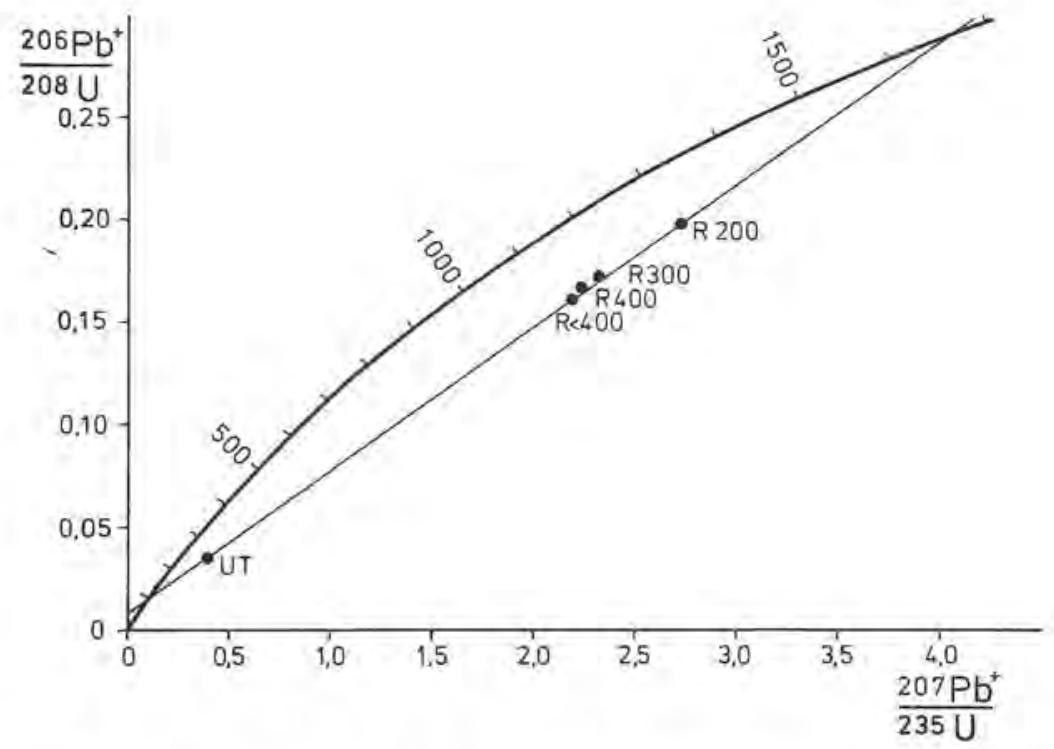

Abb. 5,-6; Discordante Zirkone (nach SILVER u. DEUTSCH 1963)

führlich beschriebene "Zwei-Ereignis-Modell" wurde erstmalig von WETHERILL (1956) abgeleitet. TILTON (1960) hat gezeigt, daß auch im Falle eínes gleichmäßigen, tber die ganze geologische Geschichte des Minerals andauernde Diffusion des radiogenen Bleies aus dem Mineral eine der "Diskordia" ähnliche lineare Beziehung zwischen den beiden $\mathrm{Pb} / \mathrm{U}$-Verhältnissen besteht.

Die Abweichung der diskordanten $\mathrm{Pb} / \mathrm{U}$-Verhältnisse von den dem wahren AIter $t_{0}$ entsprechenden Wertepaar (Abb. 5, -7) wird hier analog dem Wert $x$ beim einmaligen Bleiverlust durch die Größe $\frac{D}{2}$ bestimmt, wobei D der Diffusionskoeffizient und a der ${ }^{a}$ Radius der als kugelförmig angenommenen Mineralkörner ist. Die Probenmeßpunkte liegen auf einer zunächst gradlinigen "Diskordia", die aber nicht gradining bis zu dem zweiten Schnittpunkt $t_{1}$ fortzusetzen ist, sondern 


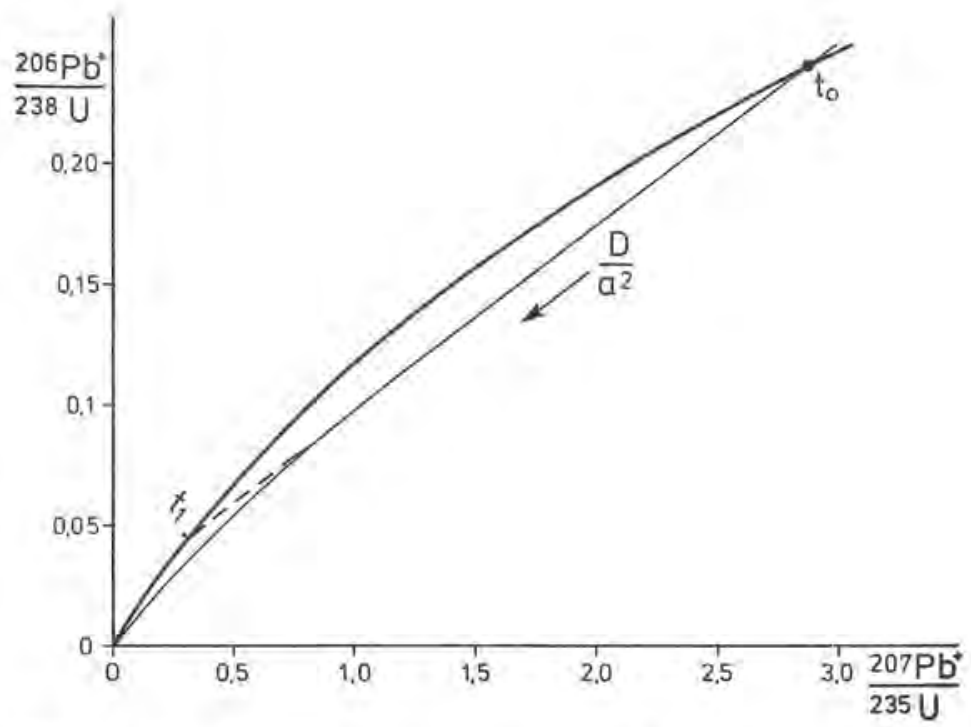

Abb. 5.-7: Die Diffusions-Discordia

die in ihrem unteren Teil zum Koordinatenanfang hin einschwenkt. In einem solchen Fall eines konstanten Diffusionsverlustes liefert der obere Schnittpunkt einer durch eine lineare Anordnung von ProbenmeBpunkten definierten Geraden das wahre Alter $t_{{ }^{\prime}}$, das durch den unteren Schnittpunkt dieser extrapolierten Geraden mit der "Concordia" erhaltene Alter $t_{1}$ hingegen hat keine geologische Bedeutung.

Diese beiden Modelle: Das Zwei-Ereignis-Modell und das Diffusionsmodell sind die einzigen etwas komplizierten Modelle, die mit einer ausreichenden $\mathrm{Zahl}$ von Proben eine Bestimmung der gesuchten Alterswerte möglich machen. Schon bei einem zweimaligen Blei- oder uranverlust, oder einer konstanten Diffusion verbunden mit einem einmaligen Verlust ist es nicht mehr möglich, aus den $\mathrm{Pb} / \mathrm{U}-$ Verhältnissen ein Alter zu errechnen. 
Die $\mathrm{Pb} / \mathrm{U}$-Methode 1 iefert also nur dann eindeutige Ergebnisse, wenn das zu datierende Mineral außer seiner primären Bildung höchstens noch eine spätere störung des Systems z.B. durch eine metamorphe Beeinflussung erlebt hat. Bei mehreren solchen Ereignissen, die zu teilweisem Verlust von Blei oder Uran gefürt haben, liegen die durch ihre $\frac{207 \mathrm{~Pb}^{+}}{235 \mathrm{U}}, \frac{206 \mathrm{~Pb}^{+}}{238 \mathrm{U}}-$ Wertepaare gekennzeichneten Meßpunkte im "Concordia"-Diagramm nicht mehr auf einer geraden Linie.

\subsection{Die $210_{\mathrm{Pb}-\text { Methode }}$}

Eine Variante der ${ }^{206} \mathrm{~Pb}^{+}-{ }^{238} \mathrm{U}$-Methode zur Datierung von uranhaltigen Mineralen hat HoUTERMANS (1951) gefunden. Wenn sich die gesamte Zerfallreihe des ${ }^{238} \mathrm{v}$ (Abb. 5. - I) in radioaktivem Gleichgewicht vefindet, so heißt das ja, daß pro Volumeneinheit des Minerals jedes der Tochterisotope des ${ }^{238} \mathrm{U}$ die gleiche $\mathrm{Zahl}$ von radioaktiven Zerfällen pro Zeiteinheit macht wie das Mutterisotop ${ }^{238} \mathrm{U}$ selbst. Die $\mathrm{Zahl}$ der Zerfälle pro Zeiteinheit $\dot{n}$ einer $\mathrm{Zahl} \mathrm{N}$ von Isotopen ist

$$
\dot{\mathrm{n}}=\lambda \mathrm{N} \text {, }
$$

wobel $\lambda$ die Zerfallskonstante des betreffenden Isotopes ist. Es gilt also für das ${ }^{210} \mathrm{~Pb}$, das auch als RaD bezeichnet wird:

$$
\dot{\mathrm{n}}_{210}=\dot{\mathrm{n}}_{238}=\lambda_{238} \cdot{ }^{238} \mathrm{U} \text {. }
$$

Eliminiert man mit dieser Beziehung die Größe ${ }^{238} \mathrm{U}$ in der ersten der Gleichungen $(5,-2)$, so erhält man für die spezifische ${ }^{210} \mathrm{~Pb}$ Radioaktivität des radiogenen Bleies 206

$$
\frac{\mathrm{n}_{210}}{206 \mathrm{~Pb}^{-}}+\frac{238}{\mathrm{e}^{238^{t}}-1}
$$


Um eine anschaulichere Vorstellung von der Altersabhängigkeit der spezifischen ${ }^{210} \mathrm{~Pb}-\mathrm{Aktivităt} \mathrm{zu}$ erhalten, betrachten wir die Vereinfachung der Formel $(5,-9)$ für junge Alter, d.h., $\lambda_{238} t \ll 1$, also einen Altersbereich $t<200 \mathrm{ma}$. Entwickelt man die Exponentialfunktion im Nenner bis zum linearen Glied, so wird Gl. $(5 .-9) \mathrm{zu}$

$$
\frac{n_{210}}{206 \mathrm{~Pb}^{+}}=\frac{1}{t}
$$

Man erhält also eine äußerst einfache Beziehung, daß die $\mathrm{Zahl}$ der ${ }^{210} \mathrm{~Pb}$ Zerfälle pro zeiteinheit und pro radiogenes ${ }^{206} \mathrm{~Pb}$-Atom gleich dem reziproken Alter (gemessen in den gleichen Zeiteinheiten wie die ${ }^{210} \mathrm{~Pb}-\mathrm{Zerfallsrate)}$ ist. Auf handliche Einheiten umgerechnet sind obige Formeln umzuschreiben in

$$
\frac{\dot{\mathrm{n}}_{210}}{{ }^{206} \mathrm{~Pb}^{+}}=\frac{.8539}{\mathrm{e}^{\lambda_{238^{t}}-1}} \quad \begin{aligned}
& \text { näherungsweise } \\
& \text { für kleine } t
\end{aligned}=\frac{5556}{t},
$$

wobei $\dot{\mathrm{n}}_{210}$ in apm (= Zerfälle pro Minute), ${ }^{206} \mathrm{pb}^{+}$in $\mu \mathrm{g}$ radiogenes ${ }^{206} \mathrm{~Pb}$ und das Alter $t$ in der Näherungsformel in $10^{6}$ Jahren einzusetzen ist. Die spezifische ${ }^{210} \mathrm{~Pb}-\mathrm{Akti}$ ivität pro $\mu \mathrm{g}{ }^{206} \mathrm{~Pb}$ ist in $\mathrm{Abb}, 5,-8$ als Funktion des Alters $t$ aufgetragen, und man sieht, daB für kleine Alter ( $t<200$ ma) die gestrichelt gezeichnete Naherungslösung (Gl, 5,-10) den Verlauf der exakten Funktion $(5 .-9)$ sehr gut wiedergibt. Der Vorteil dieser Methode im Vergleich zur Pb-u-Methode liegt darin, daß keine quantitative urananalyse mehr notwendig ist, sondern lediglich eine (nicht quantitative) $\mathrm{Ab}-$ trennung des Bleies aus dem Mineral und eine Bestimmung der zur $210 \mathrm{~Pb}$-Aktivitätsmessung gelangenden Menge des radiogenen ${ }^{206} \mathrm{~Pb}^{+}$. Denn durch chemische Trennungsvorgänge kann das Mengenverhältnis von radioaktivem ${ }^{210} \mathrm{pb}$ und radiogenem ${ }^{206} \mathrm{~Pb}$, da es sich um Isotope des gleichen chemischen Elementes han- 


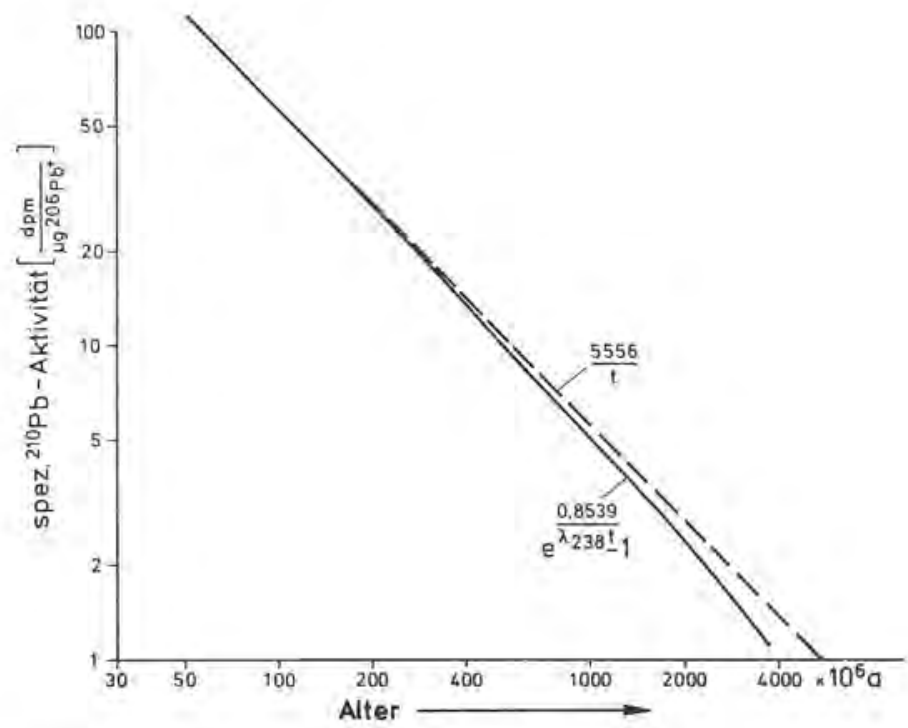

Abb, 5, -8 : Die spezifische ${ }^{210} \mathrm{pb}-$ Aktivität als Funktion des Alte

delt, nicht verändert werden, und nur dieses Verhältnis geht in die Formel $(5,-9$ bzw. 10) zur Berechnung des Alters ein. Die ${ }^{210} \mathrm{pb}-$ Methode liefert also im Fall des radioaktiven Gleichgewichtes, - und das wird für alle Pb-U-Methoden für die gesamte geologische Geschichte der zu datierenden Probe vorausgesetzt, - nichts anderes als die ${ }^{206} \mathrm{~Pb}-{ }^{238} \mathrm{U}$-Methode. Aber gerade diese Voraussetzung des radioaktiven Gleichgewichtes kann durch Vergleich dieser beiden Methoden tiberprüft werden. Würde z.B. eines der radioaktiven Zwischenisotope dex Uranreihe etwa das gasförmige ${ }^{222}$ Rn aus dem Mineral teilweise entweichen, so wird das ${ }^{206} \mathrm{~Pb}^{+}$nicht mehr an Ort des zerfallenden ${ }^{238} \mathrm{U}$-Isotopes sein, das ${ }^{206} \mathrm{pb}^{+}-{ }^{238} \mathrm{U}-$ Verhältnis und damit das sich daraus errechnende 206/238-A1ter wird zu klein sein. Im Falle eines konstanten relativen $222 \mathrm{Rn}$ Verlustes über das gesamte Alter des Minerals wird die ${ }^{206} \mathrm{~Pb}-{ }^{238} \mathrm{U}$-Methode ein zu kleines, die ${ }^{210} \mathrm{~Pb}-$ Methode hin- 
gegen das wahre Alter ergeben. Wegen der wesentlich kürzeren Halbwertszeit des ${ }^{219} \mathrm{Rn}$ aus der ${ }^{235} \mathrm{U}$-Zerfallsreihe wirkt sich ein Diffusionsverlust des gasförmigen Radons auf die ${ }^{207} \mathrm{~Pb}-$ ${ }^{235} \mathrm{U}$-Methode weit weniger aus als auf die ${ }^{206} \mathrm{~Pb}-{ }^{238} \mathrm{u}$-Methode. Dieses Beispiel mag einmal zelgen, welche Möglichkeiten dex Kontrolle der Datierungsergebnisse durch Kombination von mehreren Methoden zur Verfügung stehen.

Die Messung der Aktivität des ${ }^{210} \mathrm{~Pb}$ erfolgt nach HouTERMANS (1951) an einer wässrigen $\mathrm{Pb}$-Lösung im Flüssigkeitszählrohr und Vergleich mit einer Standardlösung bekannter ${ }^{210} \mathrm{~Pb}$ Aktivität. Hierbei wird nicht die weiche $B$-Strahlung des ${ }^{210} \mathrm{~Pb}$ direkt, sondern die härtere B-Strahlung des unmittelbaren Folgeproduktes ${ }^{210_{\mathrm{Bi}}}$ gemessen, das mit einer Halbwertszeit von 5,0 Tagen aus dem ${ }^{210} \mathrm{~Pb}$ entsteht. KULP et al. (1953) ebenso wie HŏHNDORF (1968) bestimmen die $210_{\mathrm{pb}-\text { Aktivität }}$ durch $\alpha$-Messung am ${ }^{210_{\mathrm{PO}}}$, dem Folgeprodukt des $210_{\mathrm{B} i}$.

\subsection{Die "Fission-track"-Methode}

Diese von PRICE \& WALKER (1963) erstmalig für die Datierung entdeckte Methode basiert im Gegensatz zu allen anderen bisher beschriebenen Methoden nicht auf dem radioaktiven Zerfall, sondern auf der spontanen Kernspaltung eines Isotops, hier dem ${ }^{238} \mathrm{U}$. Die $\mathrm{zah} 1$ der spontanen Kernspaltung pro Zeiteinheit $\mathrm{n}_{\mathrm{S}}$ einer Versamulung von ${ }^{238} \mathrm{v}$-Atomen ( ${ }^{238} \mathrm{U}$ repräsentiert hier eine $\mathrm{Zah} 1$ von Atomen) ist analog dem radioaktiven Zerfall:

$$
\mathrm{n}_{\mathrm{s}}=\lambda_{\mathrm{f}} \cdot{ }^{238} \mathrm{U}
$$

wobei $\lambda_{f}=8.42 \times 10^{-17} a^{-1}$, die Konstante des spontanen Kernzerfalls ist. Sie ist mehr als sieben Größenordnungen kleiner als die des radioaktiven Zerfalls. Ein paar Zahlenangaben mögen dies anschaulicher darstelien:

$1 \mathrm{\mu g}$ Uran sind $\frac{6.02 \times 10^{23}}{238 \times 10^{6}}=2.53 \times 10^{15}$ Atome,

d.h., $1 \mu \mathrm{g}^{238} \mathrm{U}$ macht nach GI. $(5,-11)$ 


$$
\mathrm{n}_{\mathrm{s}}=\lambda_{\mathrm{f}} \cdot{ }^{238} \mathrm{U}=8.42 \times 10^{-17} \cdot 2.53 \times 10^{15}=0.213 \mathrm{a}^{-1}
$$

also etwa 0.2 spontane Kernspaltungen pro Jahr.

Die $\mathrm{Z}$ ahl der radioaktiven Zerfälle der gleichen Menge ${ }^{238} \mathrm{U}$ hingegen ist

$\dot{\mathrm{n}}_{238}=238^{238} \mathrm{U}=1.537 \times 10^{-10}, 2.53 \times 10^{15}=3.9 \times 10^{5} \mathrm{a}^{-1}$

also $3.9 \times 10^{5}$ pro Jahr oder 0.74 pro Minute.

Die Methode des sog. "fission-track" = der Kernspuren-

Datierung besteht nun darin, die während der Existenzzeit,

d.i. also dem Alter $t$ des Minerals stattgefundenen Spaltungsprozesse $\mathrm{N}_{s}$ zu zählen, und es ist:

$$
N_{s}=n_{s} \cdot t=\lambda_{f} \cdot{ }^{238} u \cdot t .
$$

Diese so einfach abzuleitende Formel gilt jedoch nur, solange $n_{s}$, d.h. also nach 5.-11, daß $238 \mathrm{u}$ wăhrend der Zeit $t$ als konstant angesehen werden kann, also nur für kleine Alter $t\left(t<10^{8} \mathrm{a}\right)$, für die die Anderung der $\mathrm{zahl}$ der $238 \mathrm{U}$-Atome durch den radioaktiven Zerfall vernachlässigt werden kann. Für beliebige Alter $t$ ist $z u$ berücksichtigen, daß die Größe ${ }^{238} \mathrm{U}$ in Formel $(5,-12)$ ebenfalls eine Funktion der zeit ist und zwar ${ }^{238} \mathrm{v}(\mathrm{t})={ }^{238} \mathrm{Ue}{ }^{\lambda 238 t}$, wobei jetzt ${ }^{238} \mathrm{U}$ die heute in der Probe gefundene $\mathrm{Zahl}$ von ${ }^{238} \mathrm{U}$-Atomen und $\lambda_{238}$ die radioaktive zerfallskonstante ist. G1. (5.-12) ist dann zu ersetzen durch

$$
\begin{aligned}
& \text { t t } \\
& \mathrm{N}_{\mathrm{S}}=\int \mathrm{n}_{\mathrm{s}}(\mathrm{t}) \mathrm{dt}=\lambda_{\mathrm{f}}{ }^{238} \mathrm{U} \int \mathrm{e}^{\lambda_{238} \mathrm{t}} \cdot \mathrm{dt}=\frac{\lambda_{\mathrm{f}}}{\lambda_{238}}{ }^{238} \mathrm{U}\left(\mathrm{e}^{\lambda_{238} \mathrm{t}}-1\right) \\
& \text { o } \\
& \text { o }
\end{aligned}
$$

für $\lambda_{238} t \ll 1$ wird $(5,-13)$ zu $(5,-12)$

Ein Spaltprozess eines Uranatoms erzeugt in dem Mineral, in dem dieses zerfallende Atom sich befindet, eine Spur, die im 
Anschliff nach Atzen mit Flußsäure unter dem Mikroskop sichtbar ist.

Die Zah1 der an der Anschlifffläche sichtbaren Spuren $P_{S}$ ist gleich der $\mathrm{Zahl}$ der im Mineral vorhandenen Spaltspuren mal einem Geometriefaktor G, der die Verteilung des Urans im Mineral, die Tiefe unter der Mineraloberfläche im Vergleich zur Länge der Spur usw. berücksichtigt. Setzt man die gleiche Mineralprobe im Reaktor einer Dosis a thermischer Neutronen aus, so wird dadurch eine Spaltung der ${ }^{235} \mathrm{U}$-Atome induziert, deren $\mathrm{zahl} \mathrm{N}_{i}$ gegeben ist, durch

$$
\mathrm{N}_{i}={ }^{235} \mathrm{u} \cdot \mathrm{d} \cdot \sigma_{\mathrm{f}}
$$

wobei ${ }^{235} \mathrm{u}$ die $\mathrm{Zahl}$ der ${ }^{235} \mathrm{U}$-Atome in dem betrachteten Mineralstück und $\sigma_{f}=582 \times 10^{-24} \mathrm{~cm}^{2}$ der Wirkungsquerschnitt der Kernspaltung des ${ }^{235} \mathrm{U}$ ist. Diese induzierten Spaltungen erzeugen ebenfalis Spuren im Mineral, die auf die gleiche Weise sichtbar gemacht und unter dem Mikroskop ausgezählt werden können. Hierbel geht genau der gleiche Geometriefaktor $G$ in die $\mathrm{Zahl}$ der sichtbaren Spuren der induzierten Spaltung $P_{i}$ im Verhältnis zur Gesamtzahl $\mathrm{N}_{i}$ ein, so daß aus $(5 .-13)$ und $(5 .-14)$ folgt

$\frac{P_{S}}{P_{i}}=\frac{N_{S}}{N_{i}}=\frac{\lambda_{f}}{\lambda_{238}} \frac{238 U}{235 U} \frac{e^{\lambda_{238} t}-1}{d \cdot \sigma_{f}} \approx \frac{\lambda_{f}}{d \cdot \sigma_{f}} 137.7 \cdot t$

Es läBt sich also aus dem Verhältnis der ausgezahiten naturlichen und induzierten Spaltspuren bei bekannter Neutronenbestrahlungsdosis d das Alter $t$ aus Formel (5.-15) ausrechnen. Dieses sog. "fission-track"-Alter, dessen methodische Grundlagen sehr ausfuhrlich von WAGNER (1969) beschrieben worden sind, ist sehr erfolgreich für die Datierung von Tektiten von WAGNER (1966) und GENTNER et al. (1967) angewendet und mit Ergebnissen der K/Ar-Methode verglichen worden. Das "fission-track"-Alter ist jedoch sehr 
empfindlich gegen höhere Temperaturen. Schon eine Erwärmung auf $100^{\circ} \mathrm{C}$ uber längere Zeit verkleinert die Größe der Spuren und dadurch, daß einige ganz verschwinden, auch deren Anzahl und das Alter. STORZER \& WAGNER (1969) haben durch Aufheizungsexperimente einen zusammenhang zwischen der Verkleinerung des mittleren Durchmessers der Spuren und der Veränderung ihrer Anzahl (Abb. 5,-9) gefunden, mit der eine Korrektur durch thermische Effekte verkleinerte Spurenzahl möglich ist. WAGNER \& STORZER (1970) haben dieses Korrekturverfahren zur Datierung von permischem Pechstein bei Bozen angewendet. Die Häufigkeitsverteilung der Spurenđurchmesser (Abb. 5.-10) ist offensichtlich aus zwei Verteilungen zusammengesetzt, von denen die rechte um den gleichen Mittelwert wie die Verteilung der induzierten Spuren liegt, also offenbar thermisch nicht beeinflußt ist. Die zahl der dieser Vertellung zugehörigen Spuren liefern das Alter $t_{2}$ seit der letzten Erwärmung. Die linke Verteilung hat einen nur etwa halb so großen mittleren Durchmessex der Spuren, was nach der Korrekturkurve 5,-9 einem Spurenschwund von etwa 75 \& entspricht, d.h., die Gesamtzahl dieser spuren ist zu ver-

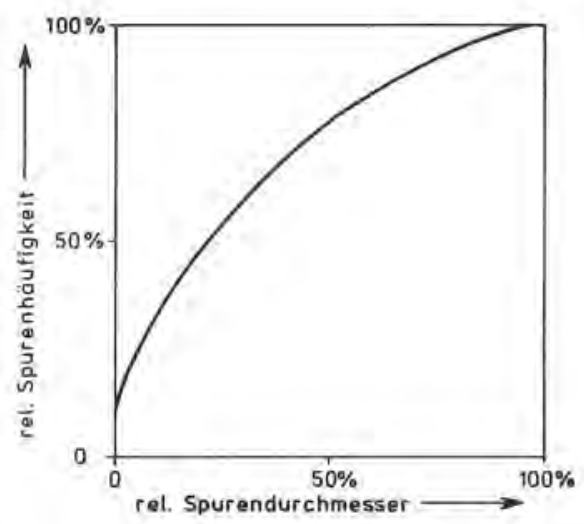

Abb. 5.-9: Spurenhäufigkeits-Durchmesserrelation bei der Spaltspurenmethode (nach WAGNER u. STORZER 1970) 


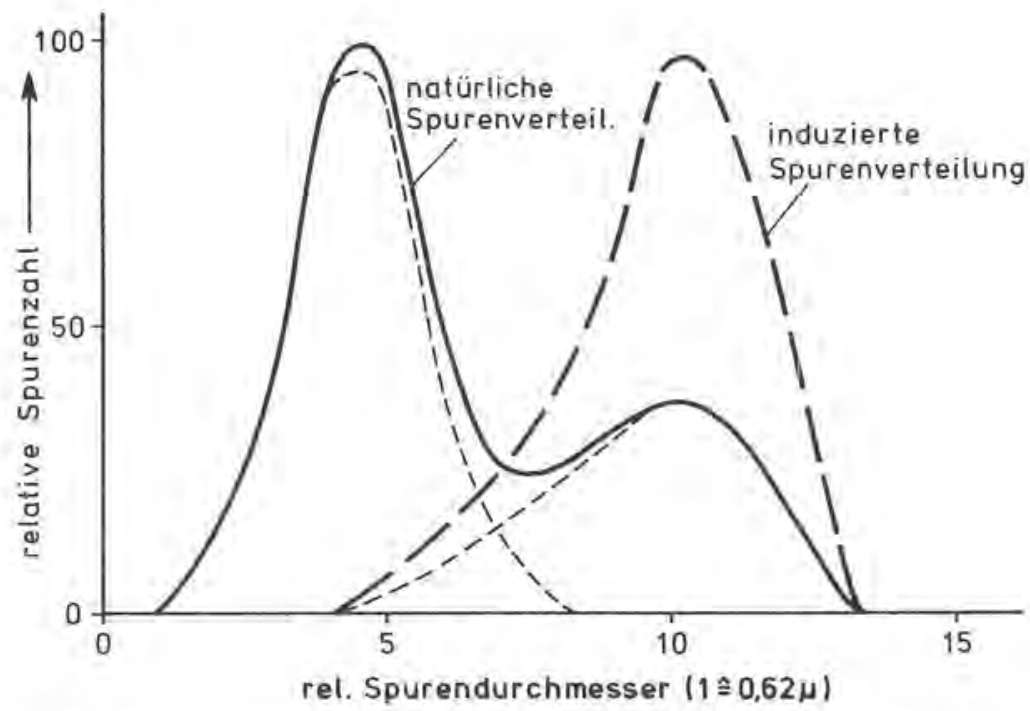

Abb. 5.-10: Spurendurchmesserspektrum bei einer Zweistufengeschichte (nach WAGNER u. STORZER 1970)

vierfachen, und das sich daraus ergebende korrigierte Alter $t_{1}$ liefert die zeit zwischen der primären Mineralbildung und der späteren Aufheizung. Die Summe von beiden ist das korrigierte Alter. Neun Proben der o.g. Pechsteine ergaben korrigierte Gesamtalter zwischen 231 und $276 \mathrm{ma}$, womit gezeigt ist, daß diese Methode nicht nur für die Datierung von jungen Altern von einigen Millionen Jahren, wie im Fall der Tektite, sondern auch für proben höheren Alters anwendbar ist. Für die Datierung von Ergußgesteinen eignen sich sehr gut zirkone, da sie einerseits eine gute stabilität der Spuren bei mäßig hohen Temperaturen haben, aber bei den hohen Temperaturen des Ergusses ( $800^{\circ} \mathrm{C}$ ) auf jeden Fall alle fruheren Spuren vollständig gelöscht werden. 
LITERATUR

HÖHNDORF, A.: Bestimmung der Halbwertszeit von ${ }^{210} \mathrm{~Pb}$. Dissertation math. nat. Fak. F.U. Berlin, 1968

KULP, J.L., BROECKER, R.W. \& ECKELMANN: Agedetermination of uranium minerals by the ${ }^{210} \mathrm{~Pb}$-Method.Nucleonics 11, 19-21, 1953.

PRICE, P.B. \& WALKER, R.M.: Fossil tracks of charged particles in mica and the age of minerals. J. Geophys. Res. $68,4847,1956$.

SILVER, L.T. \& DEUTSCH, S, : Uranium Lead Isotope Variations in Zircons. - J. Geol. 71, 721-758, 1963.

STORZER, D. \& WAGNER, G.A.: Correction of thermally Lovered Fission track Ages of Tektites. -

Earth and Plan. Sci. Lett. $\underline{5}, 463-468,1969$.

TILTON, G.R.: Volume diffusion as a mechanism for discordant Uranium-Lead ages. - J. Geophys. Res. 65 , 2933, 1960.

WAGNER, G.A.: Spuren der Kernspaltung des ${ }^{238}$ Urans. N.Jb. Miner. Abh. 110, 252-286, 1969.

WAGNER, G.A. \& STORZER, D.: Die Interpretation von Spaltspurenaltern am Beispiel von natírlichen Gläsern, Apatiten und Zirkon. - Eclogae geol. Helv. 63, $335-344,1970$.

WETHERILL, G.W.: Discordant Uranium Lead Ages I. - Trans. Am. Geophys. V. 37, 320, 1956. 


\subsection{Die Isotopenverhältnisse des gewöhnlichen Bleies}

Das gewöhnliche Blei, d.h., Blei, das in Form von reinen Bleierzen vorliegt, die kein Uran enthalten, besteht aus den vier Isotopen ${ }^{204} \mathrm{pb},{ }^{206} \mathrm{~Pb},{ }^{207} \mathrm{~Pb}$ und ${ }^{208} \mathrm{~Pb}$. Die relativen Häufigkeiten dieser Isotope sind nicht, wie das bei fast allen anderen Elementen der Fall ist, weltweit gleich. Die Ursache für diese Isotopenvariationen liegt in der Vorgeschichte des jeweiligen Blei-Erzes, d.h., ob und wie lange das Blei, bevor es als reines Bleimineral, z.B. PbS auskristallisiert ist, mit bestimmten Mengen Uran und Thorium, die radiogene $\mathrm{Blei}$-Isotope ${ }^{206} \mathrm{~Pb},{ }^{207} \mathrm{~Pb}$ und ${ }^{208} \mathrm{~Pb}$ produzieren, in einer gemeinsamen Schmelze existiert hat. Nimmt man einmal an, alle Bleierze sind durch Differentiation aus einem $\mathrm{U}$, Th und $\mathrm{Pb}$ enthaltenden Magma gebildet, so gilt für die heutige Konzentration der radiogenen Isotope ${ }^{206} \mathrm{~Pb}^{+}$und $207 \mathrm{pb}^{+}$dieses Magmas analog $\mathrm{zu} \mathrm{Gl}, 5 .-2$

$$
\begin{aligned}
& { }^{206} \mathrm{~Pb}^{+}={ }^{238} \mathrm{U}\left(\mathrm{e}^{\lambda^{2}} 238^{t} 0_{-1}\right) \\
& { }^{207} \mathrm{~Pb}^{+}={ }^{235} \mathrm{U}\left(\mathrm{e}^{\lambda} 235^{t} 0_{-1}\right),
\end{aligned}
$$

wobei to die zeitdauer der Existenz dieses "Urmagmas", also das Exdalter und ${ }^{238} \mathrm{U}$ bzw. ${ }^{235} \mathrm{D}$ die helitigen Konzentrationen dieser Isotope in diesem Magma sind. Ist aus diesem Magma vor $t$ Jahren das Blei durch Differentiation als reines $\mathrm{Pb}-$ Mineral abgetrennt worden, so sind die Pb-Mengen, die sich in der zeit zwischen $t$ und heute gebildet hätten,

$$
\begin{aligned}
{ }^{206} \mathrm{~Pb}^{+}(t) & ={ }^{238} \mathrm{U}\left(\mathrm{e}^{\lambda} 238^{t}-1\right) \\
207 \mathrm{~Pb}^{+}(t) & =235 \mathrm{U}\left(\mathrm{e}^{\lambda} 235^{t}-1\right)
\end{aligned}
$$

abzuziehen. Die zum zeitpunkt $t$ vorhandene Konzentration der $\mathrm{Pb}-$ Isotope ist also gleich der Differenz der G1, 6,-1 und 6.-2 zuzüglich den bereits zum zeitpunkt $t_{0}$ vorhandenen 
${ }^{206} \mathrm{~Pb}$ und ${ }^{207} \mathrm{~Pb}$ Konzentrationen. Es ist also:

$$
\begin{aligned}
& { }^{206} \mathrm{~Pb}={ }^{206} \mathrm{~Pb}_{\circ}+{ }^{238} \mathrm{U}\left(\mathrm{e}^{\lambda} 238^{t_{0}}{ }_{-e}{ }^{\lambda_{2}} 238^{\mathrm{t}}\right) \\
& { }^{207} \mathrm{~Pb}={ }^{207} \mathrm{~Pb}_{\mathrm{o}}+{ }^{235} \mathrm{U}\left(\mathrm{e}^{\lambda_{235^{t}} \mathrm{o}_{-\mathrm{e}}{ }^{\lambda_{2}} 25^{\mathrm{t}}}\right)
\end{aligned}
$$

oder nach Division durch das zeitlich konstante ${ }^{204} \mathrm{~Pb}$ und mit den Bezeichnungen

$$
\alpha=\frac{{ }^{206} \mathrm{~Pb}}{204 \mathrm{~Pb}} ; \quad B=\frac{207 \mathrm{~Pb}}{204 \mathrm{~Pb}} ; \quad \gamma=\frac{208 \mathrm{~Pb}}{{ }^{204} \mathrm{~Pb}}
$$

sowle

$$
\mu=\frac{238 \mathrm{U}}{204 \mathrm{~Pb}} \text { (heute); } \frac{1}{137}=\frac{235 \mathrm{U}}{238 \mathrm{U}} \text { (heute); } \kappa=\frac{232 \mathrm{Th}}{238 \mathrm{U}} \text { (heute) }
$$

erhält man:

$$
\begin{aligned}
& a-a_{0}=\mu\left(e^{\lambda_{2} 238^{t} 0_{-e}{ }^{\lambda_{2}} 238^{t}}\right) \\
& B-B_{0}=\frac{1}{137} \cdot \mu\left(e^{\lambda_{2} 235^{t} 0_{-e}{ }^{\lambda} 235^{t}}\right) \quad(6 .-4) \\
& \gamma-\gamma_{0}=\mu \cdot K\left(e^{\lambda_{2} 232^{t} 0_{-e} \lambda_{232}}\right)
\end{aligned}
$$

Diese zur zeit $t$ vorliegenden Isotopenverhältnisse änderr. sich in einem $\mathrm{Pb}$-Mineral bis heute nicht mehr, da das $\mathrm{Pb}$ von $t a b$ von den die Isotope ${ }^{206} \mathrm{pb},{ }^{207} \mathrm{pb},{ }^{208} \mathrm{pb}$ produzierenden Elementen Uran und Thorium abgetrennt ist.

\subsection{Das Holmes-Houtermans-Modell}

Falls die in den GI. $(6,-4)$ enthaltenen Konstanten $a_{0}$, ${ }_{0}$, $t_{0}, \mu$ und $k$ bekannt sind, so ware aus den gemessenen Isotopenverhältnissen $\alpha, \beta$ und $\gamma$ aus jeder der drei Gleichungen das Alter $t$ des Minerals $z u$ berechnen. 
Von besonderem Interesse sind die beiden ersten dieser drei Gleichungen, da in beiden auf der rechten seite neben to nur die Größe $\mu$, das ${ }^{238} \mathrm{U} /{ }^{204} \mathrm{~Pb}$-Verhältnis vorkormt. In einem $\alpha / B-D i a g r a m m$ erhält man mit den Konstanten

$$
a_{0}=9.56 ; \quad B_{0}=10.42 ; \quad t_{0}=4.58 \times 10^{9} \mathrm{a},
$$

die man aus uranfreien Meteoriten gewonnen hat, für verschiedene $\mu$ die sgn, "Entwicklungskurven" (in der englischen Literatur "grow-curves"), ein vom Punkt $\alpha_{0},{ }_{0}^{B}$ ausgehendes Kurvenbüschel (Abb. 6.-1). Diese Entwicklungslinien sind der geometrische ort allex $a$, $B$ Wertepaare von Bleiproben gleichen $\mu$-Wertes $\left({ }^{238} \mathrm{U} /{ }^{204} \mathrm{~Pb}\right.$-Verhältnisses), aber verschiedenen Alters $t$. Wie man aus GI. $(6,-4)$ ebenfalls ersehen kann, indem man die beiden ersten Gleichungen durcheinander dividiert, und damit eine von $\mu$ unabhăngige reine Funktion von $t$ erhält,

$$
\phi(t)=\frac{\beta^{-B_{0}}}{\alpha-\alpha_{0}}=\frac{1}{137.7} \frac{e^{+\lambda_{2}} 235^{t} o_{-}+e^{+\lambda} 235^{t}}{e^{+\lambda_{2}} 238^{t} o_{-e}+\lambda_{238} t} \quad(6 .-5)
$$

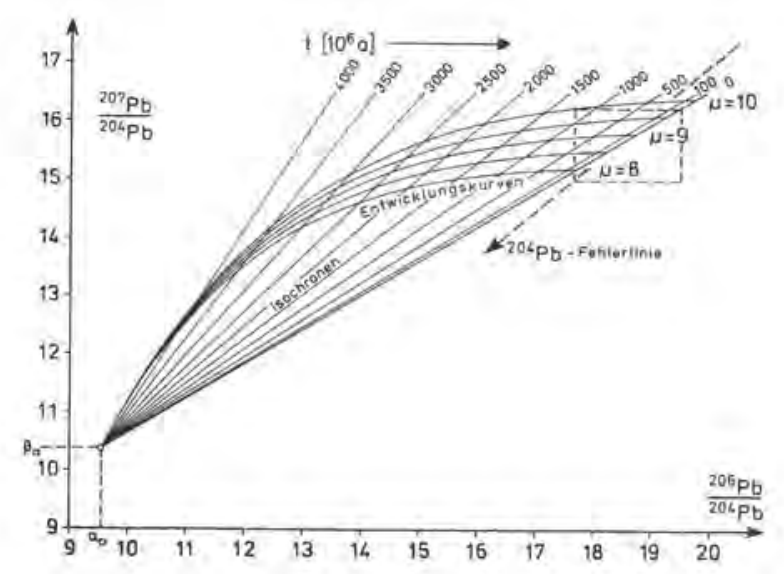

Abb. 6.-1: Die ${ }^{207} \mathrm{~Pb} /{ }^{204} \mathrm{~Pb}-{ }^{206} \mathrm{~Pb} /{ }^{204} \mathrm{~Pb}$-Entwicklungsilnien im Holmes-Houtermans Modell 


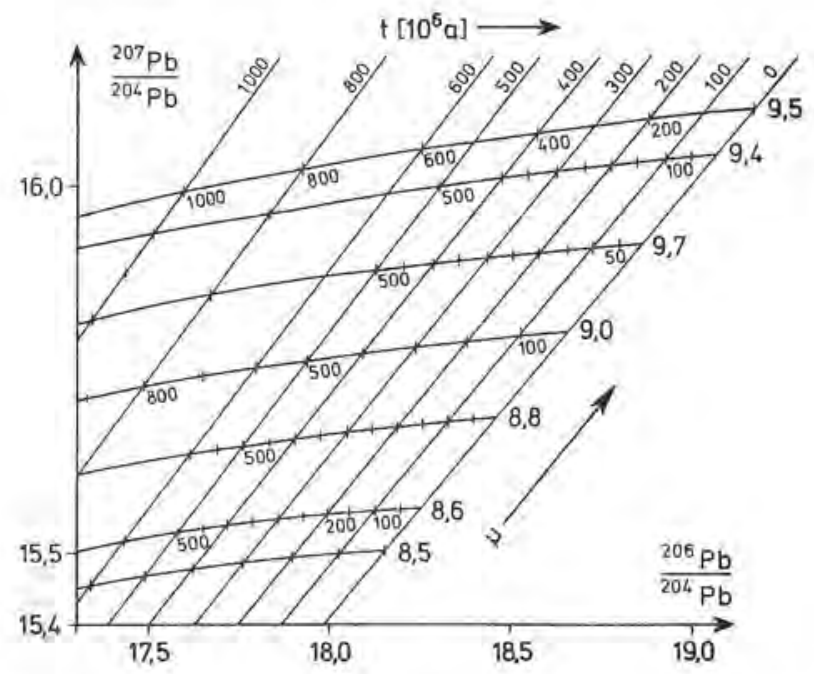

Abb. 6, -2: Ausschnittsvergrößerungen von Abb. 6.-1

liegen alle $\alpha, B$ Wertepaare von Proben gleichen Alters und beliebigen $\mu$-Werten auf Geraden, die ebenfalls durch den punkt $\alpha_{\sigma} / B_{0}$ gehen, den sog. "Isochronen". Diese durch GI. $6,-5$ gegebene Steigung $\phi(t)$ der Isochronen ist also nur eine Funktion des Alters des $\mathrm{Pb}$-Minerals, und eine Kenntnis des $\mu$-Wertes des undifferenzierten Magmas, aus dem das Bleinineral kristallisiert ist, ist nicht erforderlich.

Dieses sog. "Holmes-Houtermans"-Modell der Altersbestimmung (HOUTERMANS 1946) von Pb-Mineralen läßt alle undifferenzierte Magmen mit verschiedenen $\mu$-werten zu. Es wird lediglich vorausgesetzt, daB für das zu datierende Bleimineral eine "Einstufen-Geschichte" vorliegt, d.h., daß das Blei in der Zeit von to (dem Erdalter) und $t$, dem Bildungsalter des Pb-Minerals in einem Magma mit zwar beliebigen, aber zeitlich konstanten $\mu$ sich befunden hat, also das $\mathrm{Pb} / \mathrm{U}-\mathrm{Verhäitnis} \mathrm{in}$ diesem Magma außer durch den radioaktiven Zerfall des urans sich nicht geändert hat. In diesem Fall kann das Alter des $\mathrm{Pb}$-Minerals aus den gemessenen ${ }^{207} \mathrm{~Pb} /{ }^{204} \mathrm{~Pb}$ und ${ }^{206} \mathrm{~Pb} /{ }^{204} \mathrm{~Pb}-$ Verhältnissen nach der in $\mathrm{Abb}$. 6. -3 graphisch dargestellten 


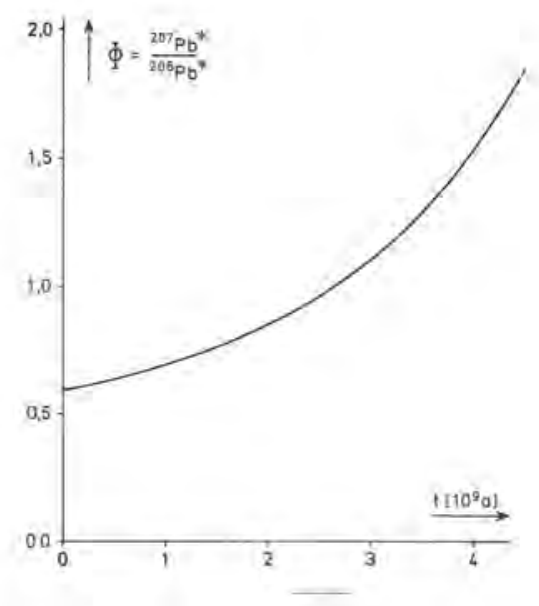

Abb. 6.-3: Die Funktion $\phi=\frac{1}{137.7} \frac{e^{\lambda 235 t} o-e^{\lambda 235 t}}{e^{\lambda 238 t} 0-e^{\lambda 238 t}}$ in Abhängigkeit von Alter $t$

Formel $(6,-5)$, oder aus den Isochronen-EntwicklungslinienDiagramen (Abb. 6,-1) bzw. für jüngere Alter in dem vergröBerten Ausschnitt der Abb, 6,-2 ermittelt werden.

Eine Auswertung von $\alpha$ - und $\beta$-Bestimmungen an vielen hundert $\mathrm{Pb}-\mathrm{Proben}$, deren Alter zwischen etwa $3000 \times 10^{6}$ a und praktisch rezent liegen, zeigt, daß alle Probenpunkte in diesem Diagramm innerhalb eines Fächers ( $A b b, 6,-8)$, dex von den Entwicklungslinien mit etwa $\mu=8.0$ und 10.0 begrenzt wird, 1 iegen, und $1 \mathrm{~m}$ Mittel um eine Entwicklungsinie mit $\mu=8.9 \mathrm{bis}$ 9.0 streuen. Es gibt aber eine ganze Reihe von Bleiproben, die ganz sicher in das "Holmes-Houtermans"-Modell nicht hineinpassen, was ganz offensichtlich für solche Proben gilt, die rechts von der Null-Isochrone llegen, d.h., denen negative Isochronen-Alterswerte zuzuordnen sind. Es sollen daher im folgenden Störungen des "Holmes-Houtermans"-Modells untersucht werden. 


\subsection{Störungen des Holmes-Houtermans-Model1s}

Lăßt man die Voraussetzung der Einstufenentwicklung fallen, nimmt man also an, daß das Blei von $t_{0}$ bis $t_{1}$ sich in einem durch $\mu_{0}$ gekennzeichneten Milieu befunden hat und vom Zeitpunkt $t_{1}$ z.B. als Gesteinsblei in einem Krustengestein mit dem Wert $\mu_{1}$ bis zum Zeitpunkt $t$ verblieben ist, un zum zeitpunkt $t$ endgultig als reines Pb-Mineral (d.h. $\mu=0$ ) auszukristallisieren, so gilt

$$
\begin{aligned}
& a=a_{0}+\mu_{0}\left(e^{\lambda_{8} t_{0}}-e^{\lambda t_{1}}\right)+\mu_{1}\left(e^{\lambda_{8} t_{1}}-e^{\lambda_{8} t}\right) \\
& B=B_{0}+\frac{\mu_{0}}{137.7}\left(e^{\lambda_{5} t_{0}} e_{-e^{\lambda_{1}} 1_{1}}+\frac{\mu_{1}}{137.7}\left(e^{\lambda_{5} t_{1}}-e^{\lambda_{5}}\right)\right.
\end{aligned}
$$

Die Entwicklungslinien sind für zwei Beispiele

$$
\text { 1.) } \begin{array}{rlrl}
t_{1}=2 \times 10^{9} \mathrm{a} \mu_{0} & =9.0 ; t=10^{8} \mathrm{a} ; \mu_{1} & =10.0 \\
\text { 2.) } & n & \mu_{1} & =8.0
\end{array}
$$

in Abb. 6. -4 dargeste11t. Für den Einstufenfal1, also

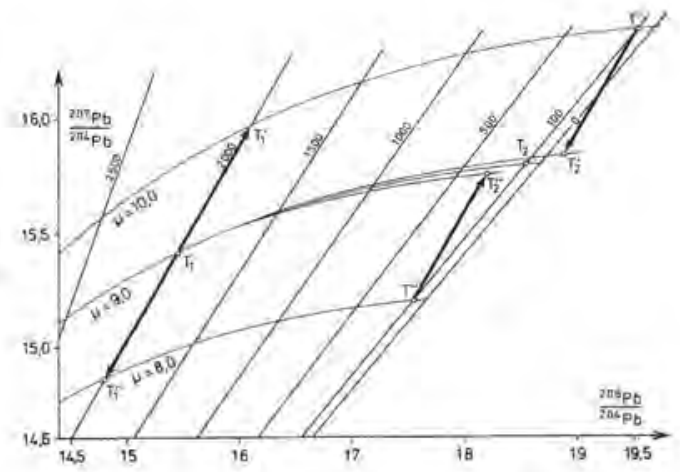

Abb. 6.-4: Graphisches Verfahren zur Darstellung von anomalem Ble

$\begin{aligned} \overline{+1} \lambda_{8} & =\lambda_{238}=\text { Zerf. Konstante des Uran-238 } \\ \lambda_{5} & =\lambda_{235}="\end{aligned}$ 
$\mu_{1}=\mu_{0}$ reduzierte sich die Gl. $(6,-6)$ zu $(6,-4)$. Die Isotopenwerte $\alpha$ und $B$ würden sich also von ${ }^{\alpha}{ }_{0}, B_{0}$ für $t=t_{0}$ ausgehend entlang der Entwicklungslinie mit $\mu=9.0$ bis dem einem Alter von $t_{1}=2 \times 10^{9}$ a entsprechenden Punkt $T_{1}$ in $\mathrm{Abb}$. 6.-4 bewegen und weiter dieser Entwicklungslinie bis zu dem einem Alter von $t=10^{8}$ entsprechenden Punkt $T_{2}$ folgen. Ist hingegen das Blei zum Zeitpunkt $t_{1}$ in eine Umgebung, deren ${ }^{238} \mathrm{U} /{ }^{204} \mathrm{pb}$-Verhältnis gleich $\mathrm{v}_{1}{ }^{1}=10.0$ ist, gebracht worden, so verläuft die Entwicklung entlang der von der einfachen Entwicklungslinie abweichenden, durch G1. 6. -6 beschriebenen Kurve von $T_{1}$ nach $T_{2}$ '.

Für das Beispiel $\mu_{1}=8.0$ entsprechend von $T_{1}$ nach $T_{2}$ ". Die Punkte $\mathrm{T}_{2}{ }^{\prime}$ (bzw. $\mathrm{T}_{2}{ }^{\prime \prime}$ ) können auch nach einem graphischen Verfahren (GEISS 1954) ermittelt werden. Man folgt der Entwicklungsinnie mit $\mu=10$ (bzw. $\mu=8$ ) bis zum schnittpunkt $T^{\prime}$ (bzW. T") mit der $t$ (hier $=10^{8} \mathrm{a}$ ) entsprechenden Isochrone. Von $T^{\prime}$ ( $\left.T^{\prime \prime}\right)$ geht man dann entlang des an $T^{\prime}$ ( $T^{\prime \prime}$ ) angesetzten, parallel verschobenen Vektors $\overline{T_{1}{ }^{\prime} T_{1}}$ (bzw. $\overline{T_{1}{ }^{\prime \prime} T_{1}}$ ), dessen Endpunkt dann der gesuchte Punkt $\mathrm{T}_{2}^{\prime}\left(\mathrm{T}_{2}{ }^{\prime \prime}\right)$ ist. Dieses graphische Verfahren folgt unmittelbar aus den G1. 6, -6, die etwas umgestellt lauten:

$$
\begin{aligned}
& a=\alpha_{0}+\mu_{1}\left(e^{\lambda_{8} t_{0}} e^{\lambda_{8} t}\right)-\left(\mu_{1}-\mu_{0}\right)\left(e^{\lambda_{8} t_{0}}-{ }^{\lambda_{8} t_{1}}\right)
\end{aligned}
$$

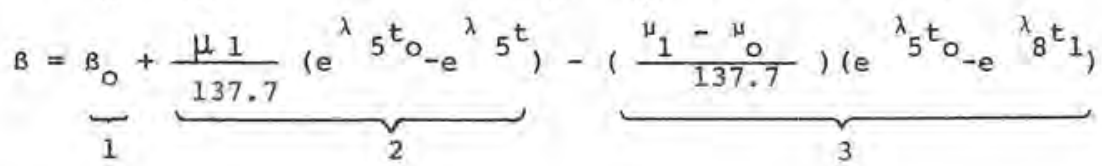

Die Terme 1 und 2 kennzeichnen den dem Alter $t$ entsprechenden Punkt auf der $\mu_{1}$-Entwicklungslinie und der Term 3 in den beiden obigen Gleichungen liefert die horizontale und vertikale Komponente des Vektors $\overline{\mathrm{T}_{I} \mathrm{~T}_{I}}$.

Die sich für diese beiden Beispiele aus den $a$ - und $\beta$-Werten

1.) $\mu_{1}=10.0 ; \alpha=18.876 ; \beta=15.840 ; \phi(t)=0.583$ entspr.t=-120ma

2.) $\mu_{1}=8.0 ; \alpha=18.187 ; B=15.754 ; \phi(t)=0.618$ entspr. $t=260 \mathrm{ma}$ 
ergebenden scheinbaren "Holmes-Houtermans-Modellalter" weichen im ersten Fall ( $\mu_{1}>\mu_{0}$ ) nach unten, im zweiten $\left(\mu_{1}>\mu_{0}\right)$ nach oben $\mathrm{ab}$. Im ersten Fall wird hier das Modellalter sogar negativ (= $120 \mathrm{ma})$. Solche, nicht in das ModeIl passende Alter bezeichnete Houtermans als "anomale" Alter und zwar für den ersten Fall $\left(\mu_{1}>\mu_{0}\right)$ als J-Typen (nach dem Vorkommen Joplin) und den zweiten Fall $\left(\mu_{1}<\mu_{0}\right)$ als B-Typen (nach dem Vorkommen "Bleiberg").

Ohne zusätzliche Informationen uber maximales oder minimales Alter des $\mathrm{Pb}$-Minerals sind zunächst nur J-Typen, die ein negatives "Alter" nach dem Holmes-Houternans-Modell liefern, klar als "anomale" Bleie zu erkennen. Gewisse zusätzliche Hinweise erhält man noch aus den Konzentrationen gewisser Spurenelemente (COHEN et al. 1958).

\subsection{Das Russel-Farguhar-Cuming (RFC) Modell}

Das Holmes-Houtermans-Modell läBt unterschiedliche U/Pb-Verhältnisse in der undifferenzierten Schmelze zu. RUSSELFARQUHAR-CUMMING (1954) gehen hingegen von der Annahme aus, daß ein homogenes Mantelmaterial mit einheitlichem $\mu$ (und einheitlichem $k$ in Gl. $(6 .-4)$ existiert, und dieser $\mu_{-}^{-}$ (und $k^{-}$) Wert muß gleich dem mittleren $\mu$-Wert des gesamten Krustenmaterial sein. Daraus folgt, das alle Bleie mit einer echten Einstufenentwicklung im $\alpha-B$-Diagram ebenso wie im $\alpha-\gamma-D i a g r a m m$ auf einer einzigen Entwicklungslinie liegen. Formt man die Entwicklungsgleichungen 6.-4 um:

$$
\begin{aligned}
& \alpha=\alpha_{0}+\mu\left(e^{\lambda t_{0}-e^{\lambda t}}\right) \\
& \alpha=\alpha_{0}+\mu\left(e^{\left.\lambda t_{0}-1\right)-\mu\left(e^{\lambda t}-1\right)}\right.
\end{aligned}
$$

und bezeichnet die ersten beiden Terme mit a,

$$
a_{0}=u\left(e^{\lambda t_{0-1}}\right)
$$


d,i. das ${ }^{206} \mathrm{~Pb} /{ }^{204} \mathrm{~Pb}$-VerhäItnis, das ein-mittleres "Mantelblei" zun heutigen zeitpunkt hat, so erhält man einen, der G1. 6,-4 entsprechenden Gleichungssatz

$$
\begin{aligned}
& \alpha=a_{0}-\mu\left(e^{\lambda_{8} t}-1\right) \\
& \beta=b_{0}-\frac{\mu}{137.7}\left(e^{\lambda_{5} t}-1\right) \\
& \gamma=c_{0}-w \cdot\left(e^{\lambda_{2} t}-1\right)
\end{aligned}
$$

mit den Konstanten

$$
\begin{array}{ll}
a_{0}=18.72 ; & b_{0}=15.82 ; \quad c_{0}=38.8 \\
\mu=9.075 ; & w=34.2 .
\end{array}
$$

Alle Bleie, die diese Voraussetzungen erfülen, müBten somit sowohl im $\alpha-\beta-$ als auch im $\alpha-\gamma-D i a g r a m a n$ auf jeweils einer einzigen Entwicklungslinie liegen. Nach RUSSEL \& FARQUHAR sind nur diese Blele, die "conformable-lead" genannt werden, Einstufentypen und alle anderen Bleie sind "anomale". Einige sehr gut untersuchte und mit vielen Probenmessungen belegte Vorkommen solchex "conformable-leads" sind in $\mathrm{Abb}, 6,-5$ und $\mathrm{Abb}$. 6. $-6 \mathrm{im}-\beta-\mathrm{bzw}$. im - -Diagram dargestellt. Solche Proben können im Prinzip mit jeder der drei Gleichungen 6. -7 datiert werden, das ${ }^{207} \mathrm{~Pb} /{ }^{204} \mathrm{~Pb}-$ Verhältnis ist jedoch während der letzten $10^{9}$ Jahre nahezu konstant, weshalb dieses Isotopenverhältnis hierzu weniger geeignet ist. Man erhält also zwei Altersgleichungen;

$$
\begin{aligned}
& t_{206}=6.51 \cdot \text { In }\left(\frac{27.8-\alpha}{9.075}\right) \\
& t_{208}=20.0 . \text { In }\left(\frac{73.00-y}{34.2}\right)
\end{aligned}
$$

Mit diesen Zahlenwerten ergibt sich $t$ in $10^{6} \mathrm{a}$. Durch Division der ersten beiden Gleichungen 6.-7 erhält man:

$$
\frac{207 \mathrm{~Pb}}{206 \mathrm{~Pb}}=\frac{15.82-0.0659\left(e^{\lambda} 5^{t}-1\right)}{18.72-9.075\left(e^{\lambda} 8^{t}-1\right)}
$$

eine Altersabhăngigkeit des ${ }^{207} \mathrm{~Pb} /{ }^{206} \mathrm{~Pb}-$ Verhältnisses der conformable Bleie. Diese Formel ist besonders angenehm, da das ${ }^{204} \mathrm{~Pb}$, das ja nur mit $\sim 1.5$ \% Häufigkeit vorhanden ist und daher nicht so genau zu messen ist wie die anderen 


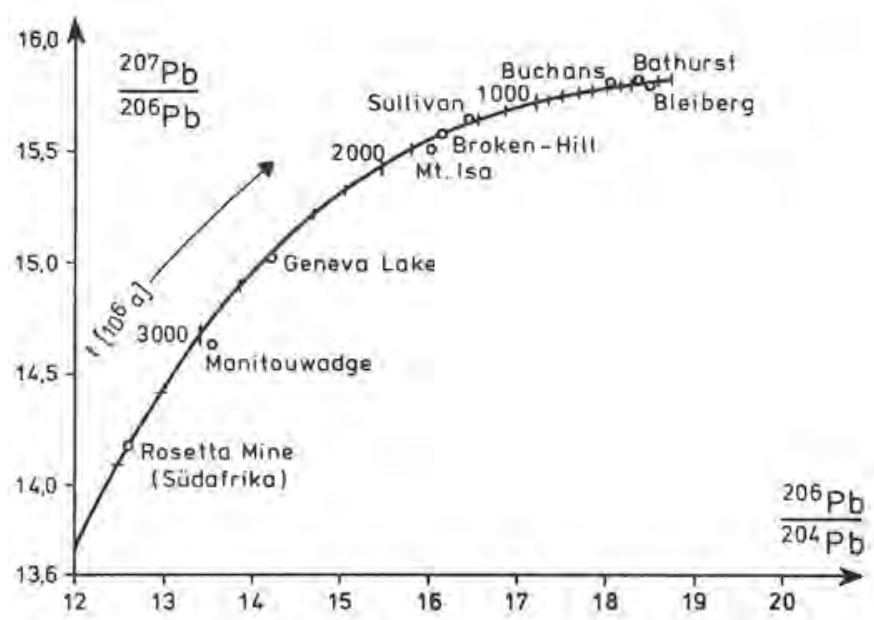

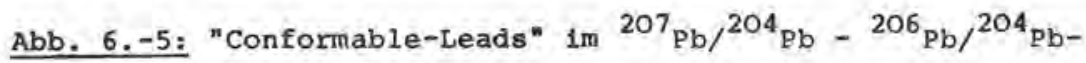
Diagramm

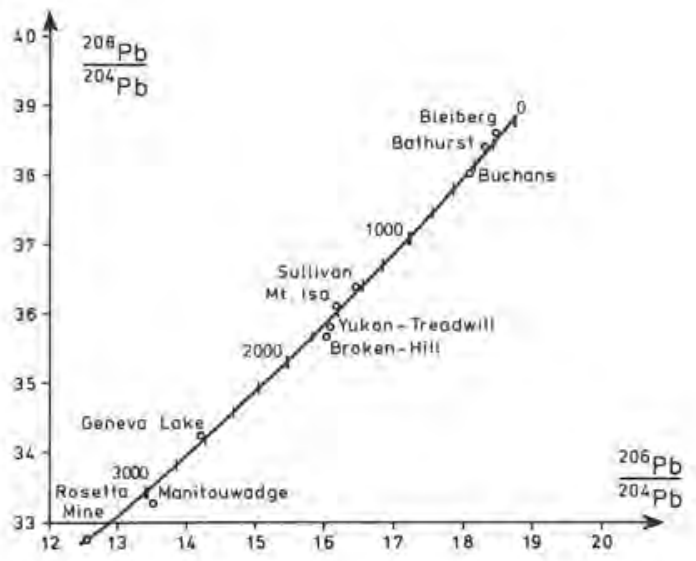

Abb. 6. -6: "Conformable-Leads" im ${ }^{208} \mathrm{pb} /{ }^{204} \mathrm{pb}-{ }^{206} \mathrm{~Pb} /{ }^{204} \mathrm{pb}-$ Diagramm 
$\mathrm{Pb}$-Isotope, nicht in die Formel eingeht. Die Abhängigkeit des ${ }^{207} \mathrm{~Pb} /{ }^{206} \mathrm{~Pb}-$ Verhältnisses vom Alter $t$ für conformable Bleie ist in $\mathrm{Abb}$. $6 .-7$ graphisch aufgetragen.

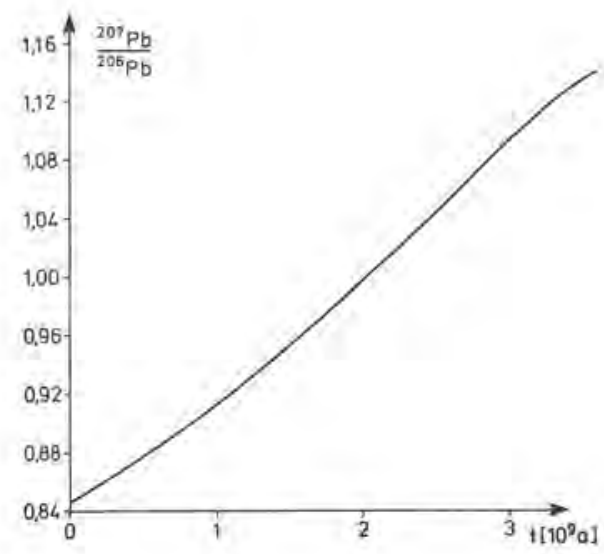

Abb. 6. -7: Das ${ }^{207} \mathrm{~Pb} /{ }^{206} \mathrm{~Pb}-$ Verhältnis als Funktion des Mineralalters

Wie man aus der Vielzahl von $\mathrm{Pb}$-Isotopenmessungen weiß, Iiegen keineswegs die meisten $\mathrm{Pb}$-Minerale auf einer solchen einheitlichen Entwicklungslinie $(\mathrm{Abb}, 6,-8)$. Nach der RFC-Theorie wären also die meisten Bleie "anomale", d.h., mindestens einmal umkristallisierte Bleie. Besonders bei älteren Isotopenmessungen kann auch eine fehlerhafte ${ }^{204} \mathrm{pb}$-Bestimmung vorliegen, die sich in gleichem Maße auf das ${ }^{207} \mathrm{~Pb} /{ }^{204} \mathrm{~Pb}-$ und das ${ }^{206} \mathrm{~Pb} /{ }^{204} \mathrm{~Pb}-$ Verhältnis auswirkt. Im $\alpha-\beta-D i a g r a m m$ würden die Punkte sich dann entlang einer in $\mathrm{Abb}, 6,-1 \mathrm{mit}$ eingezeichneten ${ }^{204} \mathrm{~Pb}$-Fehlergeraden, also einer durch den Koordinatenanfang gehenden Geraden, verschieben. Da diese Gerade für Alter < 3000 ma steiler als die Isochrone des Holmes-Houtermans-Modells verläuft, führt ein $z u$ hoher ${ }^{204} \mathrm{~Pb}-$ Wert zu einem jüngeren Modellalter und ungekehrt. 


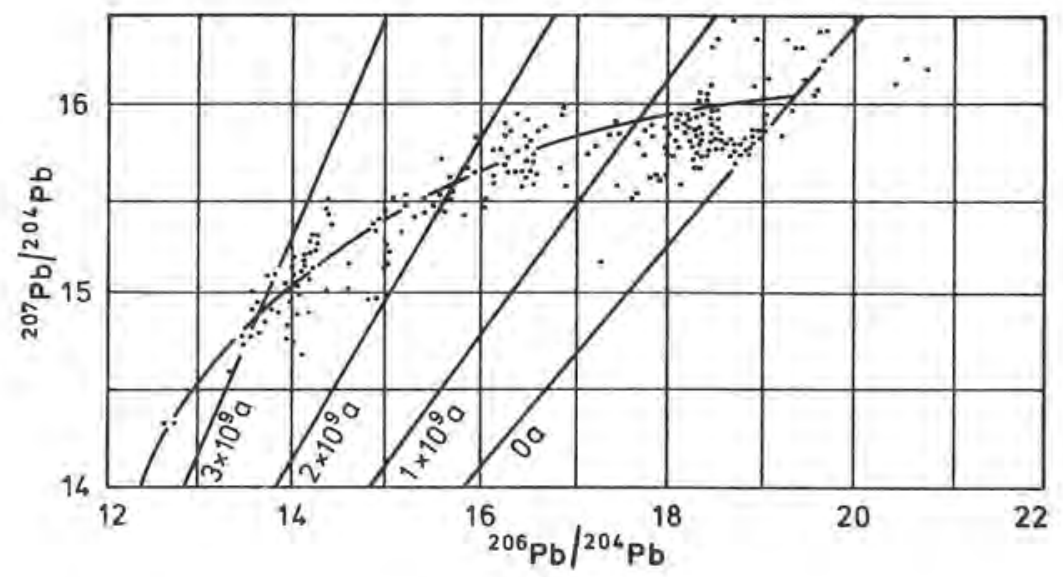

Abb. 6.-8: Verteilung der ${ }^{207} \mathrm{pb} /{ }^{204} \mathrm{pb}-{ }^{206} \mathrm{pb} /{ }^{204} \mathrm{pb}$-Werte im Entwicklungsdiagramm

Durch radiogenes Blei stark beeinflußte ganz eindeutig "anomale" Alter findet man häufig in der Nähe von Uranvorkommen. Tabelle 6.-1 zeigt einige Beispiele von "confornable" und "anomalen" Bleien. 


\begin{tabular}{|c|c|c|c|c|}
\hline & & ${ }^{206} \mathrm{~Pb} /{ }^{204} \mathrm{~Pb}$ & ${ }^{207} \mathrm{~Pb} /{ }^{204} \mathrm{~Pb}$ & ${ }^{208} \mathrm{~Pb} /{ }^{204} \mathrm{~Pb}$ \\
\hline 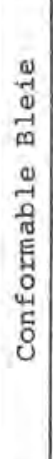 & $\begin{array}{l}\text { Bleiberg } \\
\text { Bathurst } \\
\text { Buchans } \\
\text { Sullivan } \\
\text { Mt. Isa } \\
\text { B.oken Hill } \\
\text { Geneva Lake } \\
\text { Manitouwadge } \\
\text { Rosetta Mine } \\
\text { (Südafrika) }\end{array}$ & $\begin{array}{l}18.48 \pm 0.03 \\
18.34 \pm 0.04 \\
18.18 \\
16.47 \pm 0.02 \\
16.17 \pm 0.03 \\
16.07 \pm 0.02 \\
14.20 \pm 0.05 \\
13.57 \pm 0 \\
12.60\end{array}$ & $\begin{array}{l}15.82 \pm 0.01 \\
15.82 \pm 0.02 \\
15.81 \\
15.65 \pm 0.01 \\
15.58 \pm 0.03 \\
15.50 \pm 0.02 \\
15.08 \pm 0.02 \\
14.63 \pm \\
14.18\end{array}$ & $\begin{array}{l}38.60 \pm 0.15 \\
38.38 \pm 0.08 \\
38.05 \\
36.39 \pm 0.03 \\
36.10 \pm 0.05 \\
35.80 \pm 0.03 \\
34.25 \pm 0.04 \\
33.25 \pm \\
32.72\end{array}$ \\
\hline 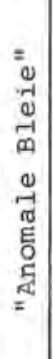 & $\begin{array}{l}\text { Joplin } \\
\text { Missouri } \\
\text { Flat River } \\
\text { (Columbia) } \\
\text { Iowa } \\
\text { Bellevue Mine } \\
\text { (Idaho) } \\
\text { Blind River }\end{array}$ & $\begin{array}{l}22.06 \\
22.07 \\
22.20 \\
22.07 \\
21.24 \\
1187.8\end{array}$ & $\begin{array}{r}15.93 \\
16.05 \\
16.18 \\
16.13 \\
16.41 \\
187.16\end{array}$ & $\begin{array}{r}41.33 \\
41.59 \\
42.45 \\
41.91 \\
42.63 \\
116.71\end{array}$ \\
\hline
\end{tabular}

Tabelle 6.-1: Pb-Isotopenverhältnisse einiger Bleivorkommen (nach RUSSEL et al. 1960) 
Zusammenfassend kann festgestellt werden:

Die Datierung von reinen $\mathrm{Pb}-\mathrm{Mineralen}$ ist, da sie nur für primär, direkt aus einem homogenen Magma gebildete Bleie mit Hilfe der beschriebenen mathematischen Modelle möglich ist, stets etwas problematisch. Dies gilt besonders für jüngere Blele (a,h., jünger als Kambrium), da Fehler in Bezug auf der Alter von \pm 200 ma die Ergebnisse nur bedingt verwendbar machen. In diesem Altersbereich soliten die $\mathrm{Pb}$-Isotopenwerte nur mehr zur Parallelisierung von verschiedenen Bleivorkommen und weniger $\mathrm{zu}$ einer direkten Altersbestimung verwendet werden. Jedoch für alle Bleiminerale ist die $\mathrm{Pb}$-Mineraldatierung, insbesondere in zusammenhang mit anderen Verfahren der Altersbestimmung von großem Nutzen. 
LITERATUR

GEISS, J.: The isotopic analysis of ordinary lead. -

Zeitschrift $\mathrm{f}$. Naturf. A/9, 218, 1954.

HOUTERMANS, F.G.: The isotope ratios in natural lead and the age of uranium.- Naturw. 33, 185-186, 1946 .

RUSSEL, R.D., FARQUHAR, R.M. , CUMMING, G.L. \& WILSON, J.T. : Dating galenas by means of their isotopic constitutions. - Trans. Am. Geoph. U. 35, $301,1954$.

RUSSEL, R.D. \& FARQUHAR, R.M.: Lead Isotopes in Geology.Intersc. Publ. Inc. New York, London, 1960. 
7. ZEITSKALA DER ERDGESCHICHTE, ALTER DER ERDE, DATIERUNG VON MONDGESTEINSPROBEN

\subsection{Die Zeitskala der Erdgeschichte}

Schon bald nachdem einige stratigraphisch gut einzuordnende, datierte Proben vorlagen, versuchte HoLMES (1947) eine "absolute Zeitskala" aufzustellen, die er später (HOLMES 1959) noch einmal verbesserte. Mit einigen Korrekturen wurde von KULP (1960) und nach einem Symposium im Jahre 1964, wo alle bisher vorliegenden radiometrischen Altersbestimungen an Proben, deren stratigraphische Position einigermaßen sicher einzugrenzen war, eingehend diskutiert worden sind, die zurzeit gültige Zeitskala (Abb. 7,-1) zusammengestellt (HARLAND et al. 1964). Die Schwierigkett in der Aufstellung einer solchen "Zeitskala", d.h., der Korrelation zwischen biostratigraphisch ermittelten Zeitabschnitten oder Epochen und radiometrisch nach den in diesem Buch beschriebenen Methoden bestimmten Jahreszahlen besteht darin, das die biostratigraphische Einstufung nur an fossilfuhrenden sedimenten direkt durchfürbar ist, die aber nicht radiometrisch datiert werden können, wăhrend die radiometrische Datierung praktisch nur an Intrusivgesteinen vorgenommen werden kann. Die bisher einzige Ausnahme bilden die Glaukonite, deren Bildungsalter in manchen Fälen mit dem Sedimentationsalter gleich zusetzen 1st, und die mit Hilfe der K/Ar-Methode datiert werden können. Glaukonite verlieren jedoch schon bei gering erhöhten Temperaturen Argon, so daB eine Glaukonitdatierung auf Gebiete beschränkt ist, für die eine postsedimentäre Erwärmung durch spätere Intrusionen ausgeschlossen werden kann. Weiterhin muß sichergestellt sein, daß nur autochtone Glaukonite und keine ungelagerten für eine Datierung herangezogen werden. DODSON et al. (in HARLAND et al. 1964) hat unter Beachtung dieser Vorschriften mit Hilfe von Glaukoniten einen wesentlichen Beitrag zur zeitlichen Unterteilung der Unterkreide und des oberen Jura geleistet. 


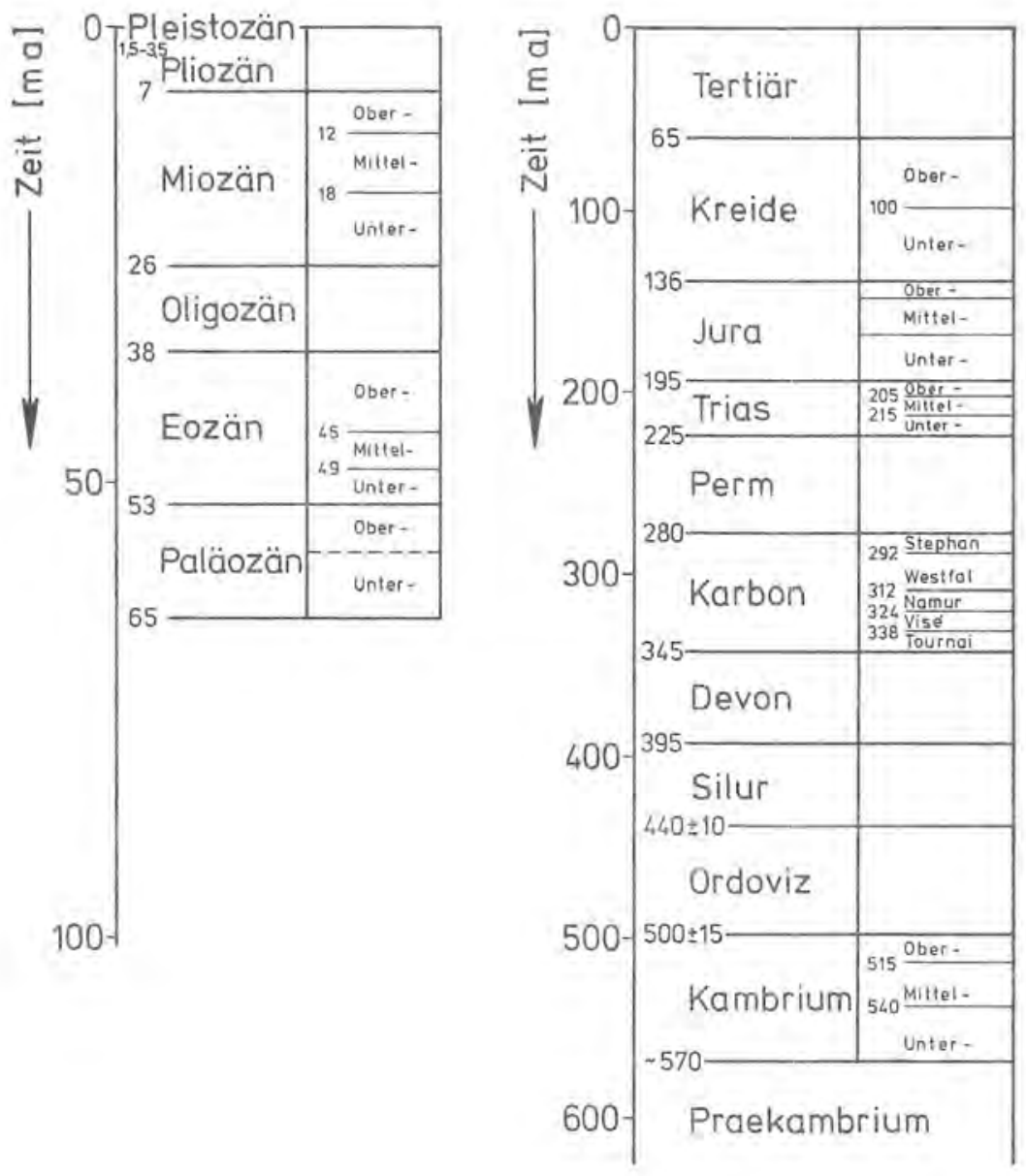

Abb. 7.-1: Die Geologische Zeitskala 
Im ubrigen ist man darauf angewiesen, datierbare Intrusivgesteine zu finden, deren stratigraphische Einstufung sich aufgrund der geologischen Gegebenheiten möglichst eng eingrenzen läßt. Solche Fälle sind naturgemäB relativ selten. Die zeltliche Gliederung, wie sie in $\mathrm{Abb}$. 7,-1 dargestellt ist, ist daher an manchen stellen noch unsicher, und neue Datierungen werden eine ständige Verbesserung und Verfeinerung dieser zeiteinteilung exbringen.

Die mit biostratigraphischen Methoden zu unterteilende zeit reicht bekanntl1ch nur bis ca. 600 ma zurück, und erst die Isotopenmethoden der Geochronologie ermöglichen es, orogene Ereignisse im Präkambrium zeitlich einzustufen. Mit Hilfe einer groBen zahl von Datlerungen im kanadischen Schild mit Altern, die bis uber 2000 ma hinausgehen, konnten einzelne "Provinzen" gleichen Alters abgegrenzt werden. Ebenso ist es erst mit Hilfe von radiometrischen Altersbestimmungen möglich, eine vorstellung von der bis in das frühe Präkambrium hineinreichenden geologischen Geschichte der alten Schilde in Südafrika, wo AIter von iber 3000 ma gefunden wurden, Skandinavien und Indien zu erhalten.

\subsection{Das Alter der Erde}

Die Frage nach dem Alter unseres planeten hat, da es von grundlegendem wissenschaftlichen Interesse ist, schon immer die , anschheit bewegt. Lediglich die Methoden, mit denen die Beantwortung dieser Frage versucht worden ist, haben sich im Laufe der leit geändert. Erzbischof USHER hat durch Addition der biblisch uberlieferten Alter der Erzväter und den Angaben uber die Schöpfungsgeschichte ein Alter von etwa 6000 Jahren ermittelt, ein Wert, dem man sehr bald berechtigte Zweifel entgegenbrachte.

Rein kernphysikalische Uberlegungen erlauben eine obere Grenze für das Alter der Erde bzw. für die Entstehung der Elemente anzugeben. Das Isotop ${ }^{235} \mathrm{U}$ mit einer ungeraden Neutronenzahl kann nicht häufiger gewesen sein, als das Isotop ${ }^{238_{U}}$ mit einer geraden Neutronenzahl, d.h., das heute $\frac{1}{137.7}$ be- 
tragende Verhältnis dieser beiden Isotope muß auch vor to Jahren, (wenn man das Erdalter mit $t_{0}$ bezeichnet) <l gewesen sein, also

$$
I>\left(\frac{235 \mathrm{U}}{238 \mathrm{U}}\right)_{t_{0}}=\frac{1}{137,7} \frac{\mathrm{e}^{\lambda 235 t_{0}}}{\mathrm{e}^{\lambda 238 t_{0}}}
$$

daraus folgt: $\quad t_{0}<6 \times 10^{9} \mathrm{a}$.

Andererseits sind mit den Isotopenmethoden ( $\mathrm{Rb} / \mathrm{Sr}, \mathrm{Pb} / \mathrm{U}$ ) bereits Gesteinsalter von $>3,3 \times 10^{9}$ a gefunden worden. Damit kann das Erdalter schon einmal mit sicherheit auf den Bereich

$$
3,3 \times 10^{9} a<t_{0}<6 \times 10^{9} a
$$

elngeengt werden.

Damit sind aber die Informationen, die man für dieses Problem aus terrestrischem Material erhalten kann, erschöpft, und man ist darauf angewiesen, zusätzliche Daten aus extraterristrischem Material zu bekommen. Das Erdalter $t_{0}$ erscheint bereits in der Formel zur Datierung von Bleimineralen (GI, 6,-4). Es ist hier eng gekoppelt mit den Werten ${ }^{a} o^{\prime}, \beta_{0}$, den Bleiisotopenverhältnissen des "Urbleis", also des Bleis vor to Jahren. Es wurde hier bereits gesagt, dab fur to ein Wert von $t_{0}=4,55 \times 10^{9}$ a einzusetzen wäre, wenn sich mit den dort angegebenen Konstanten $\alpha_{O}, B_{0}$ vernünftige Bleialter $t$ ergeben sollen.

Die Formel 6. -5 ist sehr empfindich hinsichtlich der Variation der verwendeten Konstanten bei jungen Altern $t$. $(t<200 \mathrm{ma})$. Es gilt hier

$-0.4 \cdot \Delta t_{0}-0.09 \cdot \Delta t+0,18\left(\Delta \beta-\Delta \beta_{0}\right)-0.31\left(\Delta \alpha-\Delta \alpha_{0}\right)=0$, 
wobei $\Delta t$ und $\Delta t_{0}$ in $10^{y}$ Jahren einzusetzen sind. Daraus ist $z u$ ersehen, daß eine Änderung von $t_{0}$ um $\Delta t_{0}=+50$ ma eine Änderung des Mineralalters um $\Delta t=-222$ ma ergeben würde, wenn $\alpha, \alpha, \beta, \beta$, konstant bleiben. Bei konstantem (d,h, bekanntem) $\alpha, \beta$ und $t$ hat eine Anderung von $\beta_{0}$ um $\Delta \beta_{0}=+0.1$ eine Anderung in $t_{0}$ von $\Delta t_{0}=-45 \mathrm{ma}$ bzw. bei $a_{0}$ um $\Delta a_{0}=$ +0.1 , um $\Delta t_{0}=+77.5$ ma zur Folge. Die gleichen Änderungen der Konstanten $B_{0}$ und $\alpha_{0}$ würden andererseits bei konstant gehaltenem $t$, Anderungen des Mineralalters um $\Delta t=-200$ ma im ersten und wn $+340 \mathrm{ma}$ im zweiten Fall bewirken. Die Konstanten $a_{0}, B_{0}$ und $t_{0}$ in Formel $6,-5$ sind also miteinander verknüpft, und sie sind hier mehr als parameter denn als tatsächlich mit der erforderlichen Genauigkeit emittelte MeBgrößen zu verstehen.

Aus mehreren noch undifferenzierten Proben, die seit ihrer zur zeit $t_{0}$ angenommenen Entstehung sich bis heute hinsichtlich des Urans im gleichen (aber von Probe zu Probe verschiedenen) Milieu befunden haben, also ein Differentiationsalter von $t=0$ haben, läBt sich aus der steigung der Ausgleichsgeraden nach Gl. $(6,-5)$ das Alter $t_{\circ}$ errechnen. Aus zahlreichen Meteoritenproben von PATTERSON (1956) und HESS \& MARSHALI (1960) ergibt sich eine solche "Geochrone", dex ein to von $4.6 \times 10^{9}$ a entspricht (Abb, 7.-3).

Eine Auswertung von 57 Meteoriten-Isotopenanalysen (in HAMILTON 1965) ergibt

$$
t_{0}=(4.56 \pm 0.02) \times 10^{9} \mathrm{a} .
$$

Die Annahme, diesen aus Meteoritenproben emittelten Wert auch auf das Erdalter beziehen zu können, wird dadurch gestïtzt, daß der Mittelwert des rezenten krustenbleies

$$
\begin{aligned}
& \mathrm{a}_{0}={ }^{206} \mathrm{~Pb} /{ }^{204} \mathrm{~Pb}=18.78, \\
& \mathrm{~b}_{\mathrm{o}}={ }^{207} \mathrm{~Pb} /{ }^{204} \mathrm{~Pb}=15.82
\end{aligned}
$$

ebenfalls auf dieser "Geochronen" liegen. 


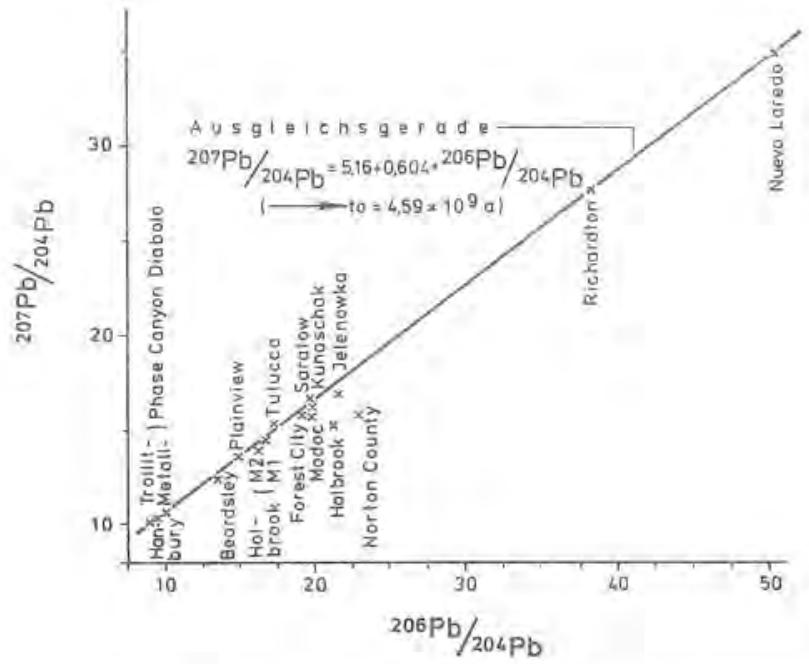

Abb. 7.-2: Die ${ }^{207} \mathrm{~Pb} /{ }^{204} \mathrm{~Pb}-{ }^{206} \mathrm{~Pb} /{ }^{204} \mathrm{~Pb}-$ Geochrone von Meteoriten-BIei

Auch die Rb/Sr-Isochronenmethode ist mit Erfolg für die Bestimmung von $t_{0}$ an Meteoriten angewendet worden. Die Isochrone liefert ein Alter von $t_{0}=4.5 \times 10^{9}$ a $(\mathrm{Abb}, 7,-4)$, Nach neueren Messungen von BOGARD (1967) erhäIt man

$$
t_{0}=(4.70 \pm 0.1) \times 10^{9} \mathrm{a} \text { (mit } \lambda_{\mathrm{Rb}}=1.39 \times 10^{-11} \mathrm{a}^{-1} \text { ) }
$$

Mit der Zerfallskonstante $\lambda=1.47 \times 10^{-11}$ a $\cdot 1$ würde sich $t_{0}=4.45 \times 10^{9}$ a ergeben. Da das Problem der Halbwertszeit des ${ }^{87} \mathrm{~Pb}$ noch nicht endgültig gelöst ist, bleibt zunächst noch dieses unsicherheit in der Rb/Sr-Datierung für das Erdalter. Auf jeden Fall kann die Ubereinstimmung mit dern Ergebnis der $\mathrm{Pb}-$ Geochrone als befriedigend angesehen werden. Die $\mathrm{k} / \mathrm{Ar}$-Datierung von Meteoriten liefert häufig wegen des Argon-Verlustes durch Aufheizen aer Meteorite, z.B, beim Eintritt in die Atmosphäre, ein zu junges Alter. Die Häufigkeitsverteilung der bisher an Meteoriten gemessenen K/Ar- 


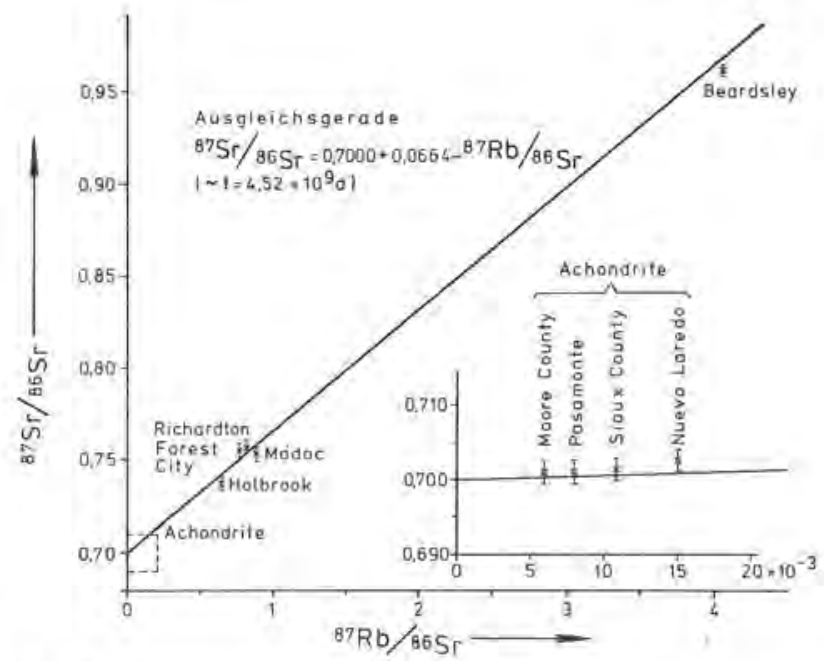

Abb. 7.-3: Rb/Sr-Isochrone von Meteoriten

Alter (Abb, 7, -4) zeigt aber eine Häufung der K/Ar-Alter bei 4.0 bis etwas über $4.5 \times 10^{9} \mathrm{a}$, ein Bild, das damit ebenfalls dem durch $\mathrm{Pb}$ - und $\mathrm{Rb} / \mathrm{Sr}$-Isochronen gefundenen Wert nicht widerspricht. Es darf daher für $t_{0}$ der wert von etwa

$$
t_{0}=4.55 \pm 0.1 \times 10^{9} \mathrm{a}
$$

angenommen werden.

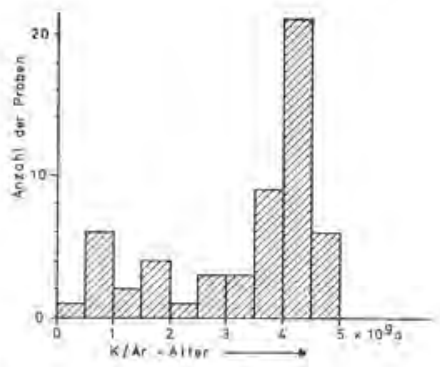

Abb. 7. 4: K/Ar-Alter von Meteoriten 


\subsection{Datierungen von Mondgestein}

Die bei der ersten bemannten Mondlandung des Apollo Il Fluges gewonnenen Proben lieferten erstmalig Material, an dem Altersbestimungen von Mondgesteinen vorgenommen werden konnten. Die Ergebnisse aller Untersuchungen sind in zahlreichen Einzelarbeiten, die im wesentlichen im Januarheft der zeitschrift Science veröffentlicht sind, zu finden. Die zur Datierung nach den verschiedenen Methoden verwendeten Proben lassen sich in vier Gruppen unterteilen:

Typ A: feinkörniges Kristallingestein

Typ B: mittelkörniges Kristallingestein

Typ C: Brekzie

Typ D: Staub

WANLESS et al. (1970) exhielt mit der K/Ar-Methode Alter von 2260 bis 3370 ma für proben des Typs A und B, Werte, die offenbar in unterschiedilcher Weise durch Argon-Verlust. verändert worden sind.

TURNER (1970) konnte mit Hilfe der ${ }^{40}$ Ar/ ${ }^{39}$ Ar-Korrektur den Argon-Verlust eliminieren und erhielt werte von $3,6-3.9 \times 10^{9}$ a für Typ A und B Gesteine.

MURTHX et al. (I970a) (1970b) fanden ein gemeinsames Rb/SrIsochronenalter von $4.42 \pm 0.12 \times 10^{9} \mathrm{a}\left(\lambda=1.39 \times 10^{-11} \mathrm{a}^{-1}\right)$ mit einem Anfangswert von $\left(\frac{87 \mathrm{Sr}}{{ }^{86} \mathrm{Sr}}\right)_{0}=0.6987 \pm 0.0001$ für die Proben vom Typ A, B, C und D. Die Streuung der Meßpunkte um diese Isochrone ubersteigt jedoch die Fehlergrenzen.

Auch COMPSTON et al. (1970) finden eine $\mathrm{Rb} / \mathrm{Sr}$-Isochrone fur alle Proben von $3.81 \pm 0.07 \times 10^{9}$ ma mit einem Anfangswert von $\left({ }^{87} \mathrm{Sr} /{ }^{86} \mathrm{Sr}\right)_{\mathrm{o}}=0.69936 \pm 0.00008$.

Eine sehr detaillierte Rb/Sr-Untersuchung wurde von PAPANASTASSIOU et al. (1970) an Ganzgesteinsproben und Mineralseparaten des Mondgesteins durchgeführt. Sechs Proben des Kristallingesteins lieferten Einzelisochronen aus Mineralfraktionen, die folgende Werte 1 ieferten $\left(\lambda=1.39 \times 10^{-11} a^{-1}\right.$ ) 


\begin{tabular}{|c|c|c|}
\hline Probe & Alter $\left(x \quad 10^{9}\right.$ a) & $\left.l^{87} \mathrm{Sr} l^{86} \mathrm{Sr}\right)_{0}$ \\
\hline 10017 & $3.59 \pm 0.05$ & $0.69932 \pm 0.00005$ \\
\hline 10044 & $3.71 \pm 0.11$ & $0.69909 \pm 0.00007$ \\
\hline 10057 & $3.63 \pm 0.02$ & $0.69939 \pm 0.00004$ \\
\hline 1058 & $3.63 \pm 0.20$ & $0.69906 \pm 0.00008$ \\
\hline 1069 & $3.68 \pm$ & $0.69929 \pm$ \\
\hline 10071 & $3.68 \pm 0.02$ & $0.69926 \pm 0.00003$ \\
\hline
\end{tabular}

Eine Ganzgesteinsisochrone dieser sechs Proben lieferte

$$
\begin{aligned}
t & =(3.76 \pm 0.12) \times 10^{9} \mathrm{a} \\
\left(\frac{87 \mathrm{Sr}}{86}\right)_{0} & =0.69914 \pm 0.00008
\end{aligned}
$$

in guter Ubereinstimmung mit den sechs internen Mineralisochronen der Einzelproben.

Im Gegensatz zu diesen Alterswerten exhielten die o.g. Autoren für Mondboden- und Staubproben und die Brekzien eine Isochrone mit $t=4.67 \times 10^{9} \mathrm{a}$, einem Wert also, der etwa gleich dem Erdalter ist. Obwohl die ${ }^{87} \mathrm{Rb} /{ }^{86} \mathrm{Sr}-$ Verhältnisse der Mondproben extrem niedrig liegen $(<0.1)$, konnten durch eine außerordentich hohe Meßgenauigkeit im ${ }^{87} \mathrm{Sr} /{ }^{86} \mathrm{Sr}$-Verhältnis von etwa $\pm 0.1 \%$ Alterswerte mit einer relativ hohen Genauigkeit erhalten werden.

Die $\mathrm{Pb} / \mathrm{U}-\mathrm{Datierungen} \mathrm{bestätigen} \mathrm{diese} \mathrm{Ergebnisse} \mathrm{weitgehend.}$ Die Analysen von TAтsumoto (1970) zelgten, daß die $U$, Th und $\mathrm{Pb}$ Konzentrationen sehr niedrig sind (U:0.16-0.87; Th:0.53-3.4; Pb:0.29-1.7 ppm), das jedoch das Blei sehr stark radiogen ist. Dieser Umstand ermöglichte detaililerte $\mathrm{U} / \mathrm{Pb}$ - und $\mathrm{Pb} / \mathrm{Pb}-I$ sotopenanalysen. Ganzgesteinsisochronen lieferten in guter Ubereinstimmung der ${ }^{208} \mathrm{~Pb} /{ }^{204} \mathrm{pb}-{ }^{232} \mathrm{Th} /$ ${ }^{204} \mathrm{~Pb},{ }^{207} \mathrm{~Pb} /{ }^{204} \mathrm{~Pb}-{ }^{235} \mathrm{U} /{ }^{204} \mathrm{~Pb}$ und ${ }^{206} \mathrm{~Pb} /{ }^{204} \mathrm{~Pb}-{ }^{238} \mathrm{U} /{ }^{204} \mathrm{~Pb}$ Isochronendarstellung Alter von

$$
4.6 \text { bis } 4.7 \times 10^{9} \text { a für Brekzie und Feinmaterial }
$$

und

3.8 bis $4.0 \times 10^{9}$ a fur das Kristallingestein. 
Interne Mineralisochronen der Proben 10012 (Kristallin) und 10084 (Feinmaterial) bestätigen die Ganzgesteinsdaten.

Ein sehr interessantes Ergebnis liefert die Concordia-Darstellung $\left({ }^{206} \mathrm{pb}^{+} / 238 \mathrm{U}\right.$ gegen $\left.{ }^{207} \mathrm{~Pb}^{+} / /^{235} \mathrm{U}\right)$, wobei zur Bestimmung des radiogenen Bleies $\mathrm{Pb}^{+}$das Urblei abgezogen wurde. Die aus dem Ganzgestein gewonnenen MeBpunkte definieren in diesem Diagramm eine "Diskordia", deren oberer Schnittpunkt mit der "Concordia" ein Alter von 4.6 bis $4.63 \times 10^{9}$ a und deren unterer Schnittpunkt 3.4 bis $3.8 \times 10^{9}$ a liefert. Die Proben des Feinmaterials liegen nahezu auf oder geringfügig über der "Concordia", sind also etwa konkordant, während die Kristallinproben unterhalb liegen, entsprechend einem Bleiverlust von $\sim 60$ of $(x \sim 0.4)$ vor $\sim 3.8 \times 10^{9} \mathrm{a}$. Zusammenfassend kann festgestellt werden, daB alle Isotopenmethoden in relativ guter Ubereinstimmung darauf hinweisen, daB dem Mond ein Primäralter von $\sim 4.6 \times 10^{9}$ a zuzuschreiben ist, d.h., ein mit dem Erd-bzw. Meteoritenalter ubereinstimmender Wert. Eine Differentiation und Bildung von Kristallingestein im Gebiet des Mare Transquillis muß vor etwa 3.7 bis $3.8 \times 10^{9}$ stattgefunden haben.

Diese ersten Ergebnisse der Datierungen von Mondgestein lassen bereits erkennen, welche weltgehenden Informationen über die geologische Geschichte des Mondes durch die Isotopenmethoden erhalten werden können, und es ist zu erwarten, daß weitere aufschluBreiche Erkenntnisse aus den Untersuchungen der Apollo 12 und 14 Proben gewonnen werden können. 
LITERATUR

BOGARD: Rb-Sr-isochron $4 \mathrm{O}_{K}-{ }^{40} \mathrm{Ar}$ ages of the Norton County achondrite. - Earth, Plan. Sci. Lett. $\underline{3}$, $179-189,1967$.

COMPSTON, W., ARRIENS, P.A., VERNON, M.J., CHAPPEL, B.W.: Rubidium-Strontium Chronology and Chemistry of Lunar Material, - Science 167, 474-476, 1970.

HAMILTON, E.I.: Applied Geochronology. - Academic Press, London, New York, 1965.

HARLAND, W.B., GILBERT-SMITH, A., WILCOCK, B.: The Phanerozoic Time Scale. - Suppl. to Quart. J. Geol. Soc. London Vol. 120s, 1964.

HESS, P.C., MARSHALL, R.R.: The isotopic compositions and concentrations of lead in some chondritic stone meteorites. - Geoch. Cosmochim. Acta 20, $284-299,1960$.

HOLMES, A.; The construction of a Geologic Time Scale. Trans. Geol. Soc. Glasgow 21, 117-152, 1947.

HOLMES, A.: A revised Geological Time Scale. - Trans. Edinb. Geol. Soc. 17, 3, 183-216, 1959.

KULP, J.A.: The Geological Time Scale. - Int. Geol. Congr. Copenhagen pt. III, 18-27, 1960.

MURTHY, R.V., SCHMITT, R.A., REY, P.: Rubidium-Strontium age and elemental and Isotopic abundances of some trace Elements in Lunar Samples. - Science 167, $476-479,1970$.

MURTHY, R.V., EVENSEN, N.M., $\operatorname{COSC}$ IO $_{1}, \mathrm{M}_{.}$R, : Distribution of $\mathrm{K}, \mathrm{R}, \mathrm{Sr}$ and $\mathrm{Ba}$ and $\mathrm{Rb}-\mathrm{Sr}$ isotopic relations in Apollc 11 lunar samples. - Proc. of Apollo 11 Lunar Cont. Vol. $\underline{2}, 1393-1406,1970$.

PAPANASTASSIOU, P.A., WASSERBURG, G.J., BURNETT, D.S.: Rb-Sr Ages of Lunar Rocks from the Sea of Transquillity. - Earth, Plan. Sci. Lett. $\underline{8}$, $1-19,1970$.

PATTERSON, C.: Age of meteorites and the earth. - Geochim. Cosmochim. Acta 10, 230, 1956.

TATSumoto, M.: Age of the moon: An isotopic study of $\mathrm{U}-\mathrm{Th}-\mathrm{Pb}$ systematics of Apollo 11 Lunar samples II, Proc. Apollo 11 Lunar Sci. Conf. Vol. 2, $1595-1612$, 1970 . 
TURNER, G.: Argon 40/Argon 39 Dating of Lunar Rock Samples. Science 167, 466-468, 1970.

WANLESS, R.K., LOVERIDGE, W.D., STEVENS, R.D. ; Age determinations and isotopic abundances measurements on lunar samples (Apollo 11). - Proc. of Apollo 11 Lunar Science Conference Vol. 2 pp. $1729-1739,1970$. 
8. RADIOMETRISCHE ALTERSBESTIMMUNGEN AM BROCKEN INTRUSIONSKOMPLEX IM HARZ ALS BEISPIEL DER INTERPRETATION DISKORDANTER MODELLALTER

(M. SCHOELL)

\subsection{Einleitung}

Seit die Methoden der radiometrischen Altersbestimungen vom physikalischen Standpunkt aus ausgereift waren und ein Mittel der geologischen Forschung wurden, war das Phänomen der diskordanten Modellalter Gegenstand sehr vieler Untersuchungen. Anhand solcher Untersuchungen wurden die prinzipiellen Grenzen der Methoden aufgezeigt, aber auch neue Möglichkeiten dex Interpretation entdeckt. Klassisches Beispiel sind hier die Arbeiten von HART (1964), JÄGER \& NIGGLI (1964) u.a.

Was sind nun diskordante Modellalter?

Wie der Name andeutet, handelt es sich um Modellalter, die (iber das MaB der Analysengenauigkeit hinaus ${ }^{+)}$unterschiedlich sind. Sie müssen dabei an einem geologischen Körper (Gesteinsprobe oder Granitstock u,a.) ermittelt worden sein. (Unterschiedliche Modellalter an verschiedenen geologischen Körpern, z.B. Granit und Nebengestein, sind natülich nicht diskordant zu nennen).

Entsprechend der oben genannten Definition gibt es verschiedene Möglichkeiten diskordanter Modellalter:

a) Unterschiede in den Modellaltern zwischen einzelnen Datierungsmethoden ermittelt an einem Mineral (vgl. JAGER (1965) und HARRE et al. (1968)).

b) Unterschiede in den Modellaltern verschiedener Minerale eine Gesteins ermittelt mit einer Methode (bestes Literaturbeispiel HART (1964)).

c) Unterschiede zwischen Gesteinsdatierung (Isochrone) und Mineralmodellalter (vgl. Kap. 3).

+) Modellalter werden melst mit einem Fehler ( $1 \sigma$ ) der sogenannten Standardabwelchung angegeben. Dies bedeutet, daB innerhalb dieser Toleranz das Ergebnis mit einer Wahrscheinlichkeit von 688 meßtechnisch wieder ermittelt werden kann. 
d) Unterschiede in den Modellaltern verschledener Korngrößen eines Minerals (HARRE et al. 1968).

In Kap. 3 sind die Voraussetzungen genannt worden, die gemacht werden müssen, damit die gemessenen Modellalter als "wahres" Alter des Gesteins betrachtet werden können. - Diskordante Modellalter entstehen somit dadurch, daß eine dieser Voraussetzungen nicht erfüllt ist.

Es hat sich gezeigt, daß häufig der Grund für diskordante Modellalter darin zu suchen ist, das der betrachtete Bereich kein geschlossenes System war, Die betrachteten Bereiche können sein

\section{1) Das Mineral \\ 2) Das Gestein}

Für beide Fälle lassen sich an Hand der Datierungen am Brocken Intrusionskomplex Beispiele anführen.

zu 1): Nehmen wir z.B. K/Ar Modellalter an Mineralen, so kann es sein, das bestimmte Minerale aufgrund ihrer ungünstigen Gittereigenschaften oder Gitterstörstellen das radiogene Argon nlcht quantitativ im Gitter halten können. Somit ergeben diese, trotz gleicher Kristallisationsgeschichte gegenüber "dichten" Mineralen unterschiedilche Modellalter (Typ b der diskordanten Alter). Anhand der K/Ar Datierungen der Glimmer aus dem Brocken Intrusionskomplex soll auf den speziellen Fall eingegangen werden, daß Mineralgemische zur Datierung vorlagen, deren Komponenten offensichtlich unterschiedliches Argonhaltevermögen aufweisen. Es soll an diesem Beispiel dargelegt werden, wie man durch geeignete Darstellung der MeBdaten solche Effekte schnell erkennen kann.

SchlieBlich wird noch die Möglichkeit gezeigt, wie man in dem speziellen Fall diskordante Modellalter rechnerisch korrigieren kann und damit zu Ergebnissen gelangt, die für die Beantwortung der geologischen Fragestellung des Alters der Brocken Intrusion herangezogen werden können. 
Zu 2) :Für das zweite Beispiel, daB das Gestein selbst ein geöffnetes System sein kann, sollen die $\mathrm{Rb} / \mathrm{Sr}$ und $\mathrm{K} / \mathrm{Ar}$ Datierungen an einem Aplitgranitgang des Brocken Granits herangezogen werden. Die in Kap, 3 dargestellten Methoden, wie man mit Hilfe des Nicolaysen Diagramms solche Homogenisierungen darstellt, können ganz analog angewendet werden.

Die folgenden Ergebnisse sind natürlich vom Standpunkt der Anwendung für die geologische Fragestellung verzerrt dargestellt. Die Betonung der Moglichkeit, das unterschiedliche Modellalter aus einem einheitlichen geologischen Komplex ermittelt werden können, soll die Aufmerksamkeit des "Verbrauchers" auf Effekte lenken, die den Begriff "absolute Altersbestimmungen" in ein richtiges Licht rücken.

\subsection{Kurze geologische Übersicht}

Der Brocken Intrusionskomplex ist ein, das geologische Bild des Mittelharzes beherrschender, komplexer Intrusionskörper, der im wesentlichen aus drei an die Oberfläche ausstreichenden Teilplutonen besteht. Diese Teilplutone sind der Harzburger Gabbro, der Oker Granit und der Brocken Granit im eigentlichen Sinne (Abb. 8. -1).

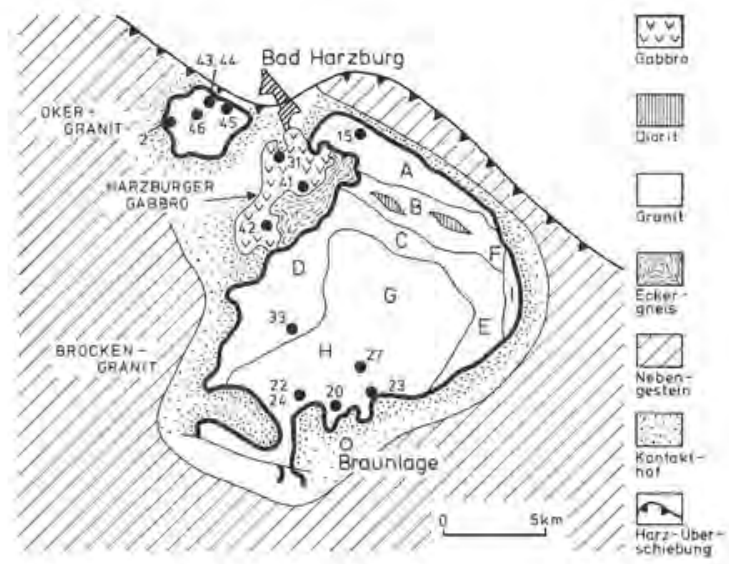

Abb. 8,-1: Der Brocken Intrusionskomplex im Harz.

Schwarze Punkte: Probennahme; Zahlen: Proben-Nr.;

Buchstaben: Teilbereiche des Brocken Granits: A: Ilsesteingranit B: Granit-Dorit Zone; C,D,E: Randgranit; G,H: Dachgranit;

I: Dorit des Ostrandes 
Es soll hier nicht auf die Einzelheiten der Geologie eingegangen werden, sie können in der sehr eingehenden Arbeit von CHROBOK (1965) für den Brocken Granit und von FUCHS (1969) für den Oker Granit nachgeschlagen werden.

Wesentlich für die Fragestellung nach dem Alter der Intrusion, d.h. im geologischen Sinne nach der Platznahme der Magmen, ist die Tatsacke, daB es sich offensichtlich um eine mehrphasige Zufuhr der Maymen handelt, wie sie in Abb. 8.-2 schematisch dargestelit ist.

Vorläuferintrusionen werden durch ultrabasische bis gabbroide Magmen gebildet; sie werden gefolgt von der normalgranitischen bis monzonitischen Hauptintrusion, die abgeschlossen wird von einem aplitgranitischen bis quarzporphyrischen Ganggefolge.

Vom geologischen Rahmen her zu schließen, ist das Maximalalter der Intrusion post unteres Namur; das Minimalalter kann durch Geländebefund lediglich als prae-Quadraten Senon angegeben werden. Regionalgeologisch und petrographische Argumente zeigen

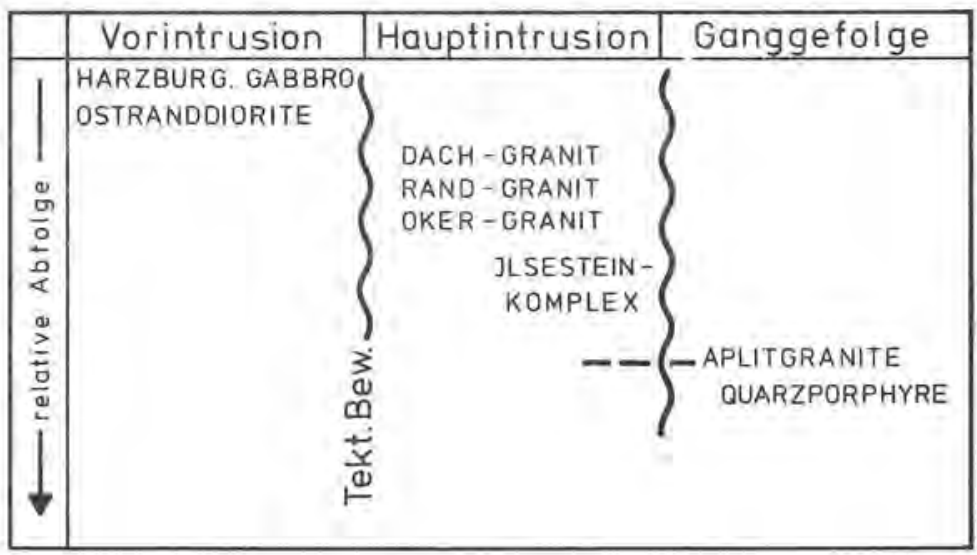

Abb. 8.-2: Schema der Intrusionsabfolge im Brocken-Intrusionskomplex 
jedoch eine starke Verwandtschaft $z$ u anderen varistischen Graniten, so daß allgemein eine Platznahme im oberkarbon angenommen wird. Auf dex anderen Seite wurde jedoch z.B. für den Ramberg Granit in neuerex Zeit durch HOPPE et al. (1965) ein Rotliegend Alter abgeleitet; es schien daher sehr wichtig zu sein, das tatsächliche Minimalalter der Brocken Intrusion festzulegen.

\subsection{K/Ar Datierungen an Biotit-Chlorit-Gemischen}

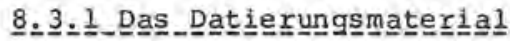

In den meisten granitischen Tiefengesteinen sind autometamorphe Umwandlungen des primären Mineralbestandes als Folge der pneumatolytisch-hydrothermalen Endphasen der Kristallisation weit verbreitet. Die spezielle Umwandiung von Biotit in Chlorit ist hierbei eine besonders auffallende Erscheinung. Sie wurde für granitische Gesteine des Moldanubikums von MULLER (1966) mineralogisch und chemisch eingehend beschrieben; für die am Brocken Intrusionskomplex bearbeiteten Proben kann die Darstellung von MULLER bis in Einzelheiten ubernommen werden. Die Aufbereitung der Glimerpräparate führt, da die Biotite mechanisch nicht von den Chloriten zu trennen sind, zu Biotit-Chlorit-Gemischen. Da Biotit und Chlorit auch sehr ähnliche magnetische Eigenschaften haben, ist eine quantitative magnetische Trennung nicht möglich. Lediglich eine Handverlesung führt schlieBllch zu reinen Biotitpräparaten.

Es lag daher nahe, diesen Umstand auszunützen und die Beimengung von Chlorit in den Blotitpräparaten systematisch zu untersuchen.

Für diesen Zweck wurden aus dem selben Gesteinsmaterial Glimmergemische verschiedener Biotit-Chlorit-Konzentration hergestellt. Da einer der Hauptvorgänge bei der Chloritisierung eine Kaliumabfuhr ist, muB sich der zunehmende Chloritgehalt in den Präparaten im Kaliumgehalt wiederspiegeln, wie es in $\mathrm{Abb}, 8,-3$ dargestellt ist. 


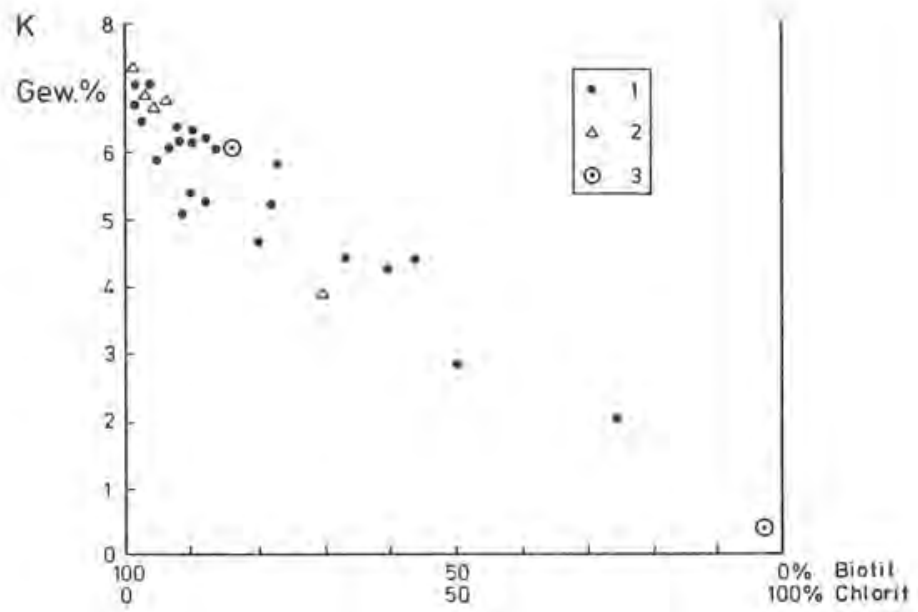

Abb, 8,-3: Kaliumgehalt der Biotit-Chlorit-Gemische in Abhängigkeit von ihrer Mineralzusammensetzung.

Punkte: Brocken-Okergranit; Dreiecke: Harzburger Gabbro; Kreis mit Punkt: Cordierit-Sillimanitgneis oberpfalz

Der Kaliungehalt wurde flamuenphotometrisch bestimut ( $\pm 0.06 \% \mathrm{~K})$, der Biotitgehalt wurde u.d.M. durch Auszählen der Chloritkörner und der chloritisierten Partien der Biotite mit dem pointcounter (kleine Schrittweite) bestimnt ( $\pm 1-2$ \% absolut).

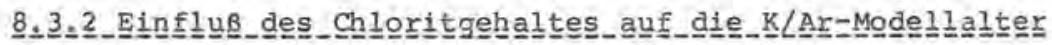

Die für die Modellalter wichtige Größe ist nun nicht der $\mathrm{K}-\mathrm{Ge}-$ halt, sondern das ${ }^{40} \mathrm{Ar} / \mathrm{K}$ Verhältnis. Es gibt somit bei der Chloritisierung drei Möglichkeiten wie sich das ${ }^{40}$ Ar/K Verhaltnis ändern kann.

1) Das Kalium wird bevorzugt aus dem Gitter entfernt, Argon verbleibt auf (evtl, anderen) Gitterplatzen (Oktaederschicht): Das ${ }^{40} \mathrm{Ar} / K$ Verhältnis, d.h. das Modellalter, muB dann mit zunehmenden Chloritgehalt ansteigen (EVERNDEN \& KISTLER 1970). Das verbleibende Argon nennt man UberschuBargon. 
2) Kalium und Argon werden in gleichen Anteilen aus dem Gitter entfernt: Das ${ }^{40} \mathrm{Ar} / \mathrm{K}$ Verhältnis bzw. das Modellalter verändert sich nicht.

3) Argon wird bevorzugt aus dem Gitter entfernt, evtl. entlang der Gitterstörstellen eines Restkalium im Chloritgitter: Das ${ }^{40} \mathrm{Ar} / \mathrm{K}$ Verhältnis und damit das Modellalter müßte mit zunehmendem Chloritgehalt abnehmen.

Die hier aufgezeigten Möglichkeiten sind in Abb. 8,-4 graphisch dargestellt.

Da das ${ }^{40} \mathrm{Ar} / \mathrm{K}$-Verhältnis ein Maß für das Modellalter ist, folgt für die Darstellung in $A b b .8,-4$, daB mit zunehmender Steigung einer Geraden (Ursprung im O-Punkt) ein gröBeres Modellalter angezeigt wird. Daraus folgt die Darstellung der oben genannten Möglichkeiten. Es sei als Beispiel nur Version 1 diskutiert: Kalium wird bevorzugt aus dem Gitter entfernt. der Punkt "Reiner Biotit" wandert in Richtung des Pfeiles 1 nach Maßgabe der Chloritbeimischung (bzw. der Kalium-Abgabe).

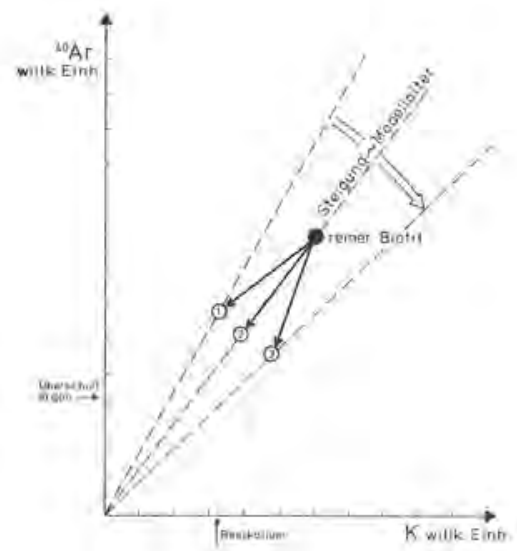

Abb. 8, -4 : Schematische Darstellung der Chlorttisierung reiner Biotite im ${ }^{40} \mathrm{Ar} / \mathrm{K}-\mathrm{Di}$ agramm, Erläuterung im Text. 
Eine Gerade durch den dadurch neu entstehenden Punkt und den Ursprung hat eine größere Steigung als die ursprüngliche Gerade "Reiner Biotit"-Ursprung. Das $40 \mathrm{Ar} / \mathrm{K}$ Verhältnis wurde also gröBer und somit das Modellalter, das wir in dieser Probe ermittelt hätten. Ganz analog ist die Erläuterung für die Fä1le 2 und 3. Die Größen "Uberschußargon" una "Restkalium" sind Korrekturgrößen, die man durch Extrapolation erhält. Im Falle des "Uberschußargons" im Chlorit z.B. kommt ihm die Bedeutung $z u$, daß es der Argon-Gehalt ist, für den kein Kalium mehr im Glimmer vorhanden ist.

Aus der $\mathrm{Abb}, 8,-4$ geht überdies ein für $\mathrm{K} / \mathrm{Ar}$ Datierungen wichtiger Zusammenhang hervor: Es muß nachgewiesen werden, $\mathrm{da} B$ bei unterschiedichem Kaliungehalt yon Mineralen,z,B. Biotiten oder Hellglimmern etc., eine Regressionsgerade $\mathrm{im}{ }^{40} \mathrm{Ar} / \mathrm{K}$ Diagramm durch den Ursprung geht. Da sonst ünterschiede in den Modellaltern keine geologische Bedeutung haben, sondern möglicherweise als Präparations fehlex anzusehen sind.

Wie in Abb. 8,-4 können wir nun die MeBergebnisse aus dem Brocken Intrusionskomplex darstellen. Beschränken wir uns hierbei zunächst auf eine Probe, deren MeBergebnisse in Abb, 8. -5 dargestellt sind. Auf der linken Seite sind die Ergebnisse in der bekannten Weise, ${ }^{40} \mathrm{Ar}$ in Abhängigkeit vom Kallumgehalt der Gemische, abgetragen. Die Linearität der Abhängigkeit dieser MeBgrößen bedeutet, daß zwischen ${ }^{40}$ ArGehalt und $\mathrm{K}$-Gehalt eine gute Korrelation besteht. Wie die Extrapolation auf einen (fiktiven) Ar-Gehait von 0 zeigt, handelt es sich offensichtlich um den Fall 3 der in Abb. 8. -4 diskutierten Möglichkeiten.

Was bedeutet nun die Extrapolation? Betrachten wir hierzu $\mathrm{Abb}$. 8. $-5 \mathrm{~B}$. Hier ist die mineralogische $\mathrm{z}$ usammensetzung, d.h. der Chloritgehalt der Gemische ebenfalls in Abhängigkeit vom Kalium-Gehalt gesetzt. Durch Extrapolation erhalten wir die K-Gehalte der reinen Endglieder der Mischungsrethe. Im Falle des Chlorits ist dieser $K_{C}=0.4 \%$. Denselben Wert erhalten wir auch durch Extrapolation in Abb. 8, $-5 \mathrm{~A}$. Daraus können wir 


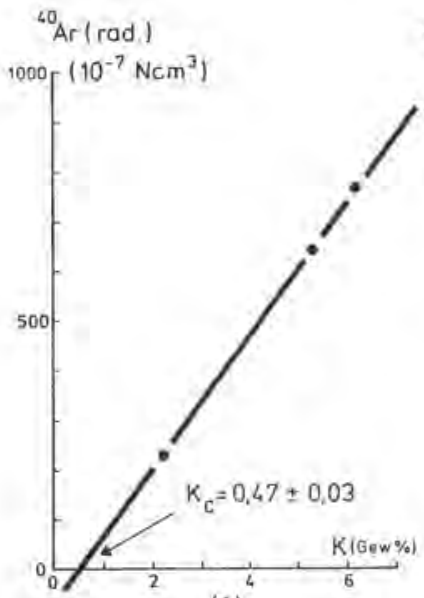

(A)

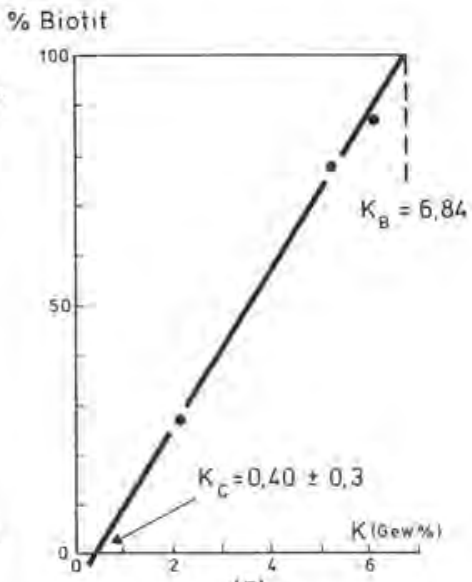

(B)

Abb. 8. -5: Abhängigkeit (A) des ${ }^{40}$ Ar-Gehaltes und (B) des Chloritanteils jeweils vom Kaliumgehalt an Gemischen der Probe 2

schlieBen, daB wir in Abb. 8. $-5 B$ einen $K_{c}$-Wert erhalten, der einem Kaliungehalt in der Probe entspricht, dem kein Zerfallsargon zukomnt (ein bezüglich der Datierung inertes Kalium), und durch den Vergleich mit $A b b$. 8, $-5 B$ zeigt sich, daB dies der Restkaliumgehalt der Chlorite sein mus.

Dieser Zusammenhang ist nicht nur für eine Probe nachgewiesen. In $\mathrm{Abb}$. 8, -6 sind die Ergebnisse aller datierten Proben zusammengestellt. Es wurden hier Regressionsgeraden errechnet unter Berücksichtigung der MeBfehler der Einzelwerte. Alle Geraden, für die einzelnen Massive gesondert dargestellt, ergeben den an der Einzelprobe oben diskutierten Zusammenhang, weil die Meßwerte innerhalb ihrer Fehler auf diesen Geraden liegen. Da die Geraden nicht duxch den Ursprung gehen (ArVerlust), werden die ${ }^{40} \mathrm{Ar} / \mathrm{K}$ Verhältnisse kleiner je geringer der $\mathrm{K}$-Gehalt der Proben wird, wie es in Abb. 8,-7 dargestellt ist. 


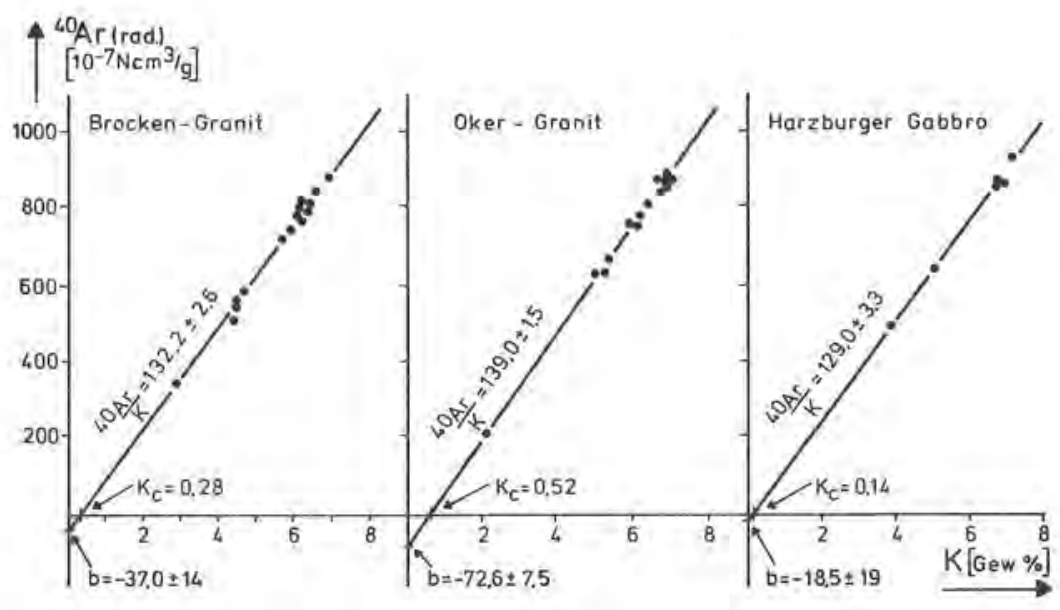

Abb. 8. -6: Die Argon Isotopenanalysen dargestellt im $40_{\mathrm{Ar} / \mathrm{K}-}$ Diagramm; aufgegliedert nach den einzelnen Massiven Anders gesprochen, wir erhalten aus einem Intrusionskomplex jedes beliebige Modellalter, was geologisch natürlich sinnlos ist und damit erhebt sich die Frage nach deren Korrektur.

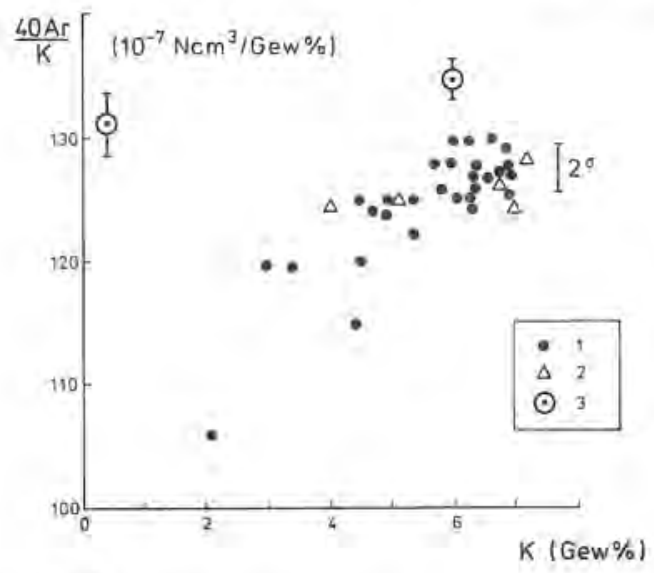

Abb. 8.-7: Die ${ }^{40} \mathrm{Ar} / \mathrm{K}$-Verhältnisse der Biotit-Chlorit-Gemische in Abhängigkeit vom Kaliumgehalt 


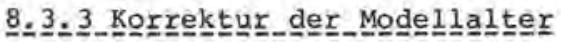

Am einfachsten kann das Prinzip der Korrektur wieder an Hand von $\mathrm{Abb}, 8,-5$ dargelegt werden. Aus $\mathrm{Abb}, 8,-5 \mathrm{~B}$ läBt sich leicht ersehen, daß man aus den $K$-Gehalten der Gemische (sie seien mit $K_{B C}$ bezeichnet) die K-Gehalte der reinen Endglieder der Mischungsreihe $\left(\mathrm{K}_{\mathrm{B}}\right.$ und $\mathrm{K}_{C}$ ) durch Extrapolation ermitteln kann. Wenn wir den Anteil an Biotit im Gemisch mit b, den des Chlorit mit $c$ bezeichnen, so ergibt sich durch einfache Verhältnisbildung in $\mathrm{Abb}$. 8. $-6 \mathrm{~B}$

$$
\mathrm{K}_{\mathrm{B}}=\frac{\mathrm{K}_{\mathrm{BC}}-{ }^{K_{C}}}{\mathrm{~b}}+\mathrm{K}_{\mathrm{C}}
$$

oder

$$
K_{B}=\frac{K_{B C}-{ }^{K_{C}}(1-b)}{b}
$$

Wenn wir nun folgende Indizes einfuhren

$$
\begin{aligned}
& B=\text { Biotit } \\
& B C=\text { Gemisch } \\
& C=\text { Chlorit }
\end{aligned}
$$

so gilt für das Modellalter des Gemisches

$$
M^{\prime}=\frac{{ }^{40}{ }_{\mathrm{Ar}}}{\mathrm{K}_{\mathrm{BC}}}
$$

Für das Modellalter des reinen Biotits muB der Anteil des Chlorits im Gemisch berücksichtigt werden und beträgt

$$
M=\frac{{ }^{40}{ }_{\mathrm{Ar}_{\mathrm{B}}}}{\mathrm{D} \cdot \mathrm{K}_{\mathrm{B}}}
$$

Da das Restkalium im Chlorit kein Argon einfürt (Abb. 8,-6A) gilt:

$$
{ }^{40}{ }_{\mathrm{Ar}} \mathrm{BC}=40_{\mathrm{Ar}}
$$


und somit labt sich das Modellalter korrigieren

$$
M=M^{\prime} \frac{K_{B C}}{b \cdot K_{B}}
$$

mit der ersten Gleichung folgt dann:

$$
M=M^{\prime} \quad \frac{K_{B C}}{K_{B C}-K_{C}(1-b)}
$$

In einer Fehlerrechnung läßt sich zeigen, daß der Fehler der Korrektur klein bleibt im Vergleich zum MeBfehler, wenn man für Gehalte $\leq 30$ \& Chlorit korrigiert.

In $\mathrm{Abb}, 8,-8$ ist der Effekt der Korrektur dargestellt.

Die unkorrigierten Modellalter uberdecken einen Bereich von 301 - $248 \mathrm{Ma}$, wogegen sich die korrigierten Werte im Bereich zwischen 302 - 288 Ma bewegen.

Die Mittelwerte der korrigierten Modellalter können aus Tab. 8. -1 entnommen werden.

Tab. 8. $-1:$

\begin{tabular}{|c|c|c|c|c|c|}
\hline & gew. Mittel & & $\begin{array}{l}\text { Ler d. } \\
\text { zelmsg. }\end{array}$ & $\begin{array}{l}\text { Fehler d. } \\
\text { Mittels }\end{array}$ & $\begin{array}{l}\text { Anzahl d. } \\
\text { Messgn. }\end{array}$ \\
\hline Brocken-Granit & 294.6 & \pm & 3.3 & \pm 0.9 & 12 \\
\hline Oker-Granit & 295.4 & \pm & 3.2 & 0.9 & 14 \\
\hline Harzb, Gabbro & 293.4 & \pm & 3.0 & 1.3 & 5 \\
\hline Alle & 294.7 & \pm & 3.2 & \pm 0.6 & 31 \\
\hline
\end{tabular}

Mittelwerte der korrigierten $\mathrm{K} / \mathrm{Ar}$ Modellalter der Biotite

Diese Werte der Tab. 8,-1 sollen später für die geologische Interpretation herangezogen werden. 


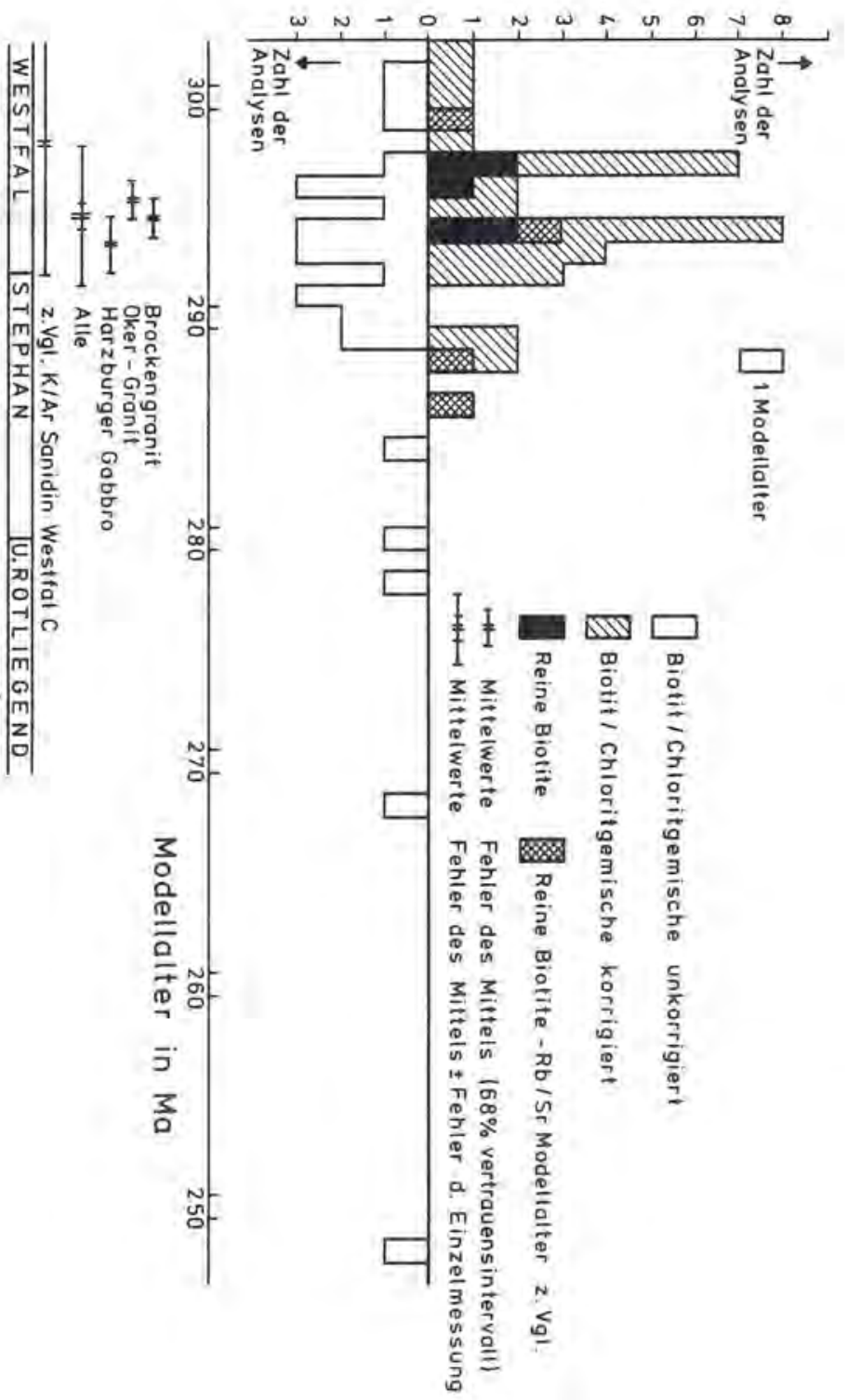

Abb. 8. -8: Häufigkeitsverteilung der K/Ar Modellalter der Biotit-Chlorit-Gemische.

Unten: Unkorrigierte Werte; Oben: Auf 100 \% Biotit korrigierte Werte. Zum Vergleich sind die $\mathrm{Rb} / \mathrm{Sr}$ Modellalter von 4 reinen Blotiten eingetragen. 


\subsection{Die $\mathrm{Rb} / \mathrm{Sr}$ Datierungen}

\subsection{1_Gesamtgesteinsanalysen_am_Brocken=_und_oker Granit}

Um den in der Einleitung genannten zweiten Fall diskordanter Modellalter zu behandeln, müssen zunächst die Ergebnisse der Datierungen an granitischen bis dioritischen Gesteinen des Oker- und Brocken Granits diskutiert werden.

Die Gesteine wurden so ausgewählt, daß ein möglichst weiter Berelch in den $\mathrm{Rb} / \mathrm{Sr}$ Verhältnissen uberstrichen wird. Da Diorite $\mathrm{Sr}$ reich und $\mathrm{Rb}$ arm sind und Granite dagegen $\mathrm{Sr}$ arm und $\mathrm{Rb}$ reich (vgl. Abb. 8. -9), war die Auswahl nicht schwierig.

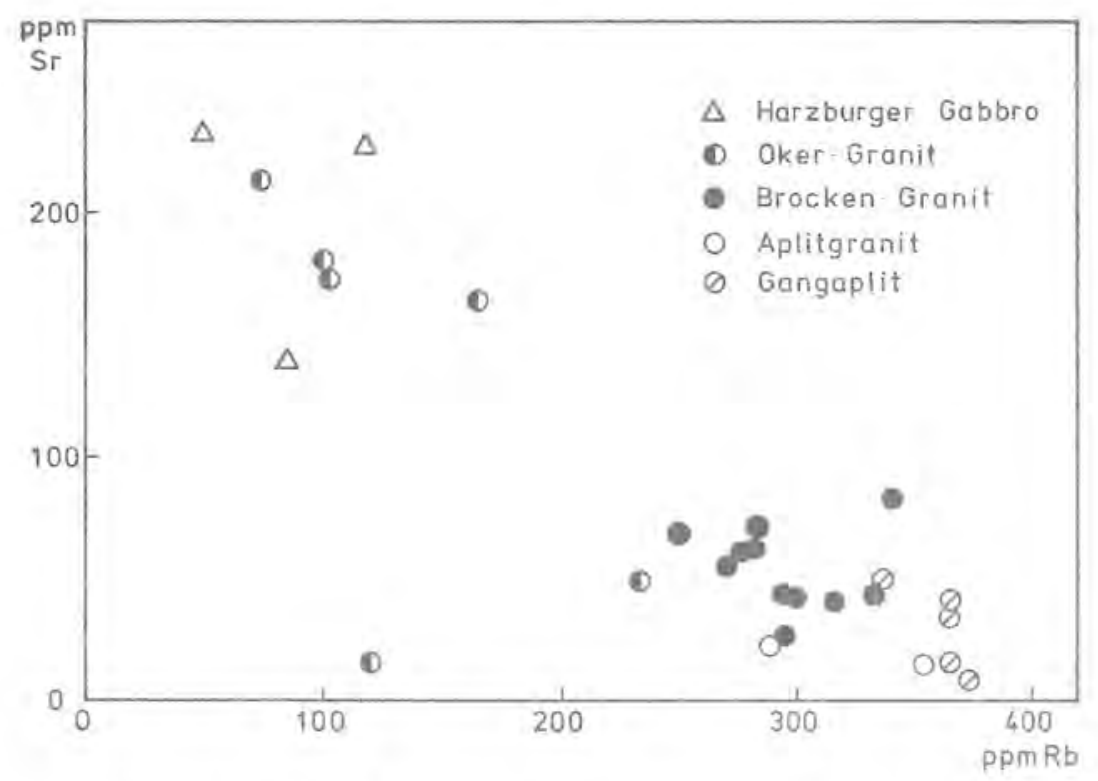

Abb. 8. -9: $\mathrm{Rb}-$ and $\mathrm{Sr}-$ Gehalt der Magmatite des Brocken Intrusionskomplexes 
Die Darstellung der Isotopenanalysen im Nicolaysen Diagramm ( $\mathrm{Abb}, 8,-10$ ) zeigt, daB die Proben von Oker Granit und Brocken Granit innerhalb threr Meßfehler auf einer Geraden liegen.

Die gesondere Berechnung der Daten des Okergranits ergab dieselbe Steigung der Geraden. Die errechneten Modellaltex und die ${ }^{87} \mathrm{Sr} /{ }^{86} \mathrm{Sr}$ Anfangswerte der Isochronen wurden in Tab. 8. -2 zusammengestellt.

Tab. 8. -2 :

Modellalter und Anfangswerte der Gesamtgesteinsisochronen

\begin{tabular}{|c|c|c|}
\hline & $\begin{array}{l}\text { Modellalter } \\
\text { in Ma }\end{array}$ & $\begin{array}{l}{ }^{87} \mathrm{Sr} /{ }^{86} \mathrm{Sr} \\
\text { Anfangswert }\end{array}$ \\
\hline Brocken-Granit & $288 \pm 12$ & $0.712 \pm 0.004$ \\
\hline Oker-Granit & $285 \pm 13$ & $0.714 \pm 0.002$ \\
\hline $\begin{array}{l}\text { Brocken- und } \\
\text { oker-Granit }\end{array}$ & $285 \pm 6$ & $0.713+0.001$ \\
\hline
\end{tabular}

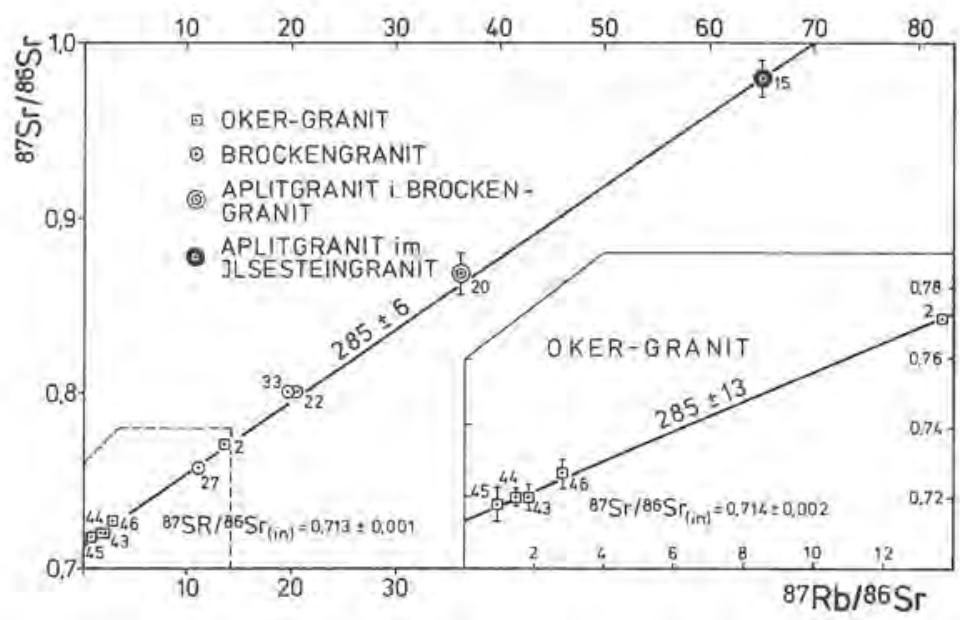

Abb. 8.-10: Die $\mathrm{Rb}$ - und $\mathrm{Sr}$-isotopenanalysen an Gesamtgesteinen, dargestellt im Nicolaysen-Diagramm 
Ubereinstimmend $\mathrm{mit}$ den $\mathrm{K} / \mathrm{Ar}$ Datierungen an den Blotiten zeigen Brocken- und Oker Granit gleiche Modellalter. Der

Systematische Unterschied zwischen $\mathrm{Rb} / \mathrm{S} x$ Gesamtgestein und K/Ar-Biotit-Modellaltern sollte nicht überbewertet werden.

1) Mußsten die MeBergebnisse mindestens $3 \sigma$ (Fehlerbalken in $\mathrm{Abb}$. 8. -15) auseinanderliegen, um statistisch signifikant verschieden zu sein.

2) Bestehen zwischen der $\mathrm{Rb} / \mathrm{Sr}$ und $\mathrm{K} / \mathrm{Ar}$ Methode noch systematische Unsicherheiten (Zerfallskonstante), so daß bei Meßfehlern von ca. 1 g der unterschied $10-15 \%$ sein mulbte, um signifikant zu sein.

\section{4_2_Gesamtgesteinsanalyse_an_einem_Aplit_granitt_Gang_als

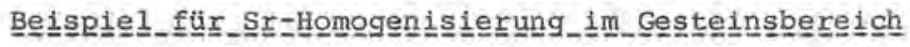

$\underline{8} \cdot \underline{4} \cdot \underline{2} \cdot \underline{1}$ Kurze petrographische Beschreibung.

Der Aplitgranit tritt im rötlich-grauen Brockengranit als heller, leukokratex \pm scharf begrenzter Gang auf. Hauptcharakteristikum ist seine chemische und mineralogische zonierung, wie es sehr deutlich aus den Variationsdiagramen in Abb, 8,-11 hervorgeht.

Man erkennt die Zonierung besonders klar am ( $\mathrm{K}$, Na) Feldspatund Plagioklasgehalt, der sich jeweils im Rb-bzw. Sr-Gehalt der elnzelnen Bereiche widerspiegelt. Entsprechend stark vari-

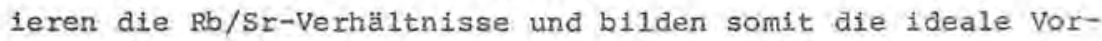
aussetzung für Isotopenanalysen. 

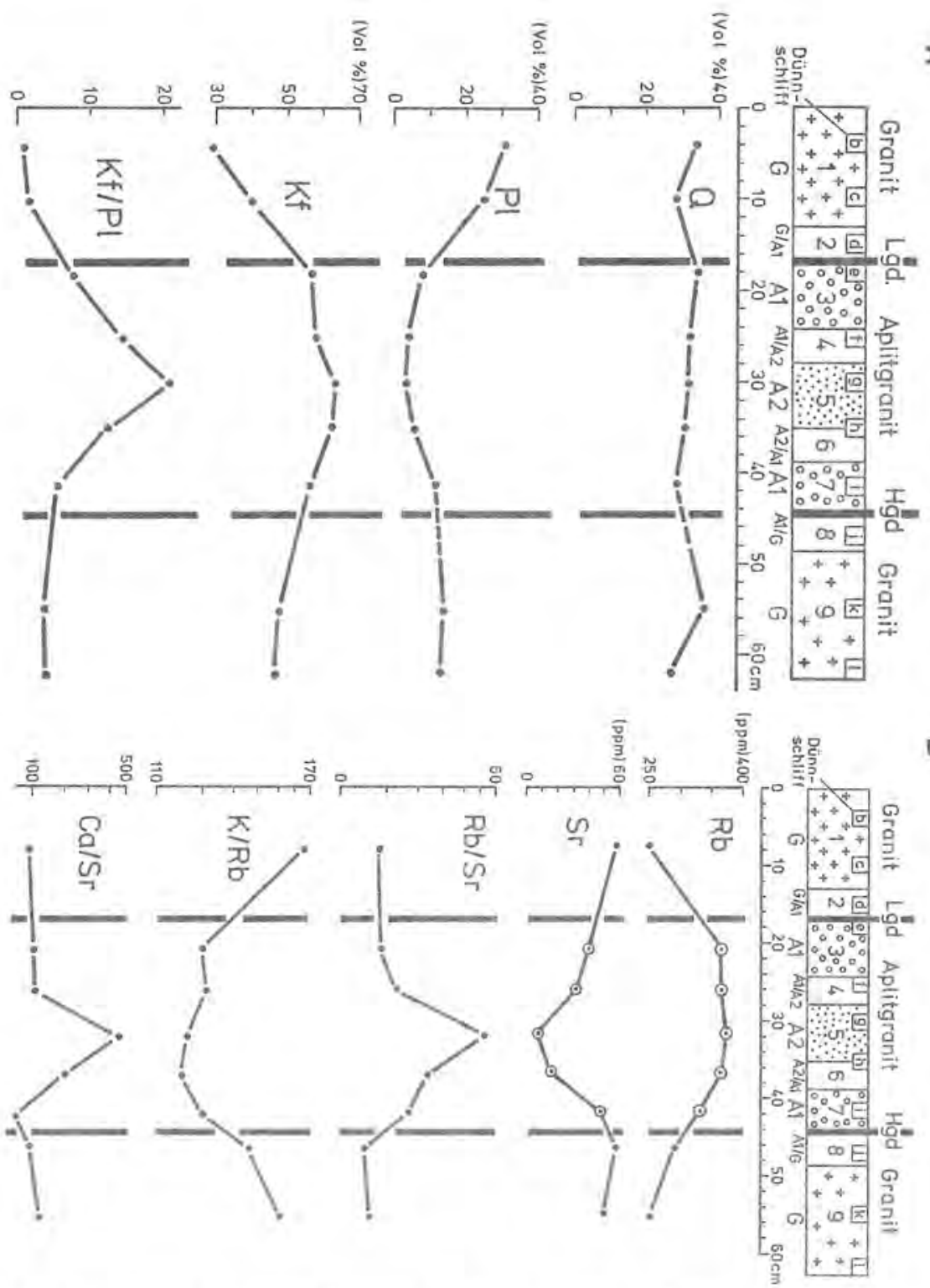

Abb. 8,-11: Varlationsdiagram (A) der mineralogischen und

(B) der chemischen Zusamensetzung des Aplitqranitganges una seines Nebengesteins.

Q) Quarz; P1: Plagioklas; Kf: (K, Na) Feldspat 


\subsection{A1lgemeines zur Sr-Homogenisier.ung_1m Gesteinsbereich}

Unter Sr-Homogenisierung im Gesteinsbereich versteht man eine Neuvertellung des radiogenen und gewöhnlichen Strontiums zu irgend einem Zeitpunkt nach der Kristallisation des Gesteins. In Kap. 3 wurde die Sr-Homogenisierung am Beispiel MineralGestein gezeigt (Abb, 3, -2). So wie ein Mineral auf kurze Distanz im Gesteinskörper eine Anomalie darstellt bezüglich der Elemente $\mathrm{Rb}$ und $\mathrm{Sr}$, so stellt auch meist ein leukokrater Gestelnskörper in einem Granit eine deraxtige Anomalie dar. Die notwendige Voraussetzung füx die Registrierung von Homogenisierungen sind diskrete, meBbare unterschiede im $\mathrm{Rb}$ - bzw. Sr-Gehalt dex betrachteten Körper, etwa in verschiedenen Mineralen eines Gesteins. Hinzu komt, daß nach Ergebnissen von Isotopenanalysen von JÄGER (1970) saure Sr arme Gesteine sehr empfindlich auf Effekte reagieren, die Sr-Homogenisierungen hervorrufen.

In $A b b ., B,-12$ sind zwei Möglichkeiten schematisch dargestellt.

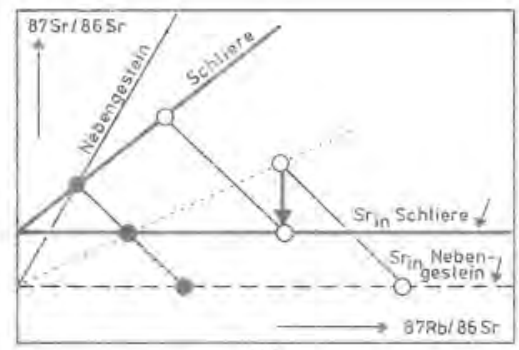

(A)

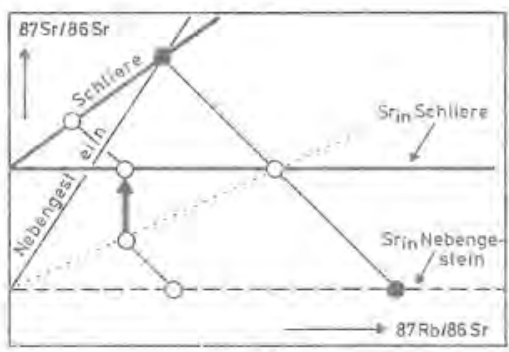

(B)

Abb. 8,-12: Schematische Darstellung der Sr-Homogenisierung zwischen Nebengestein und Gesteinsschileren oder

-gang: (A) Schlieren Sr-reicher, (B) Sr-ärmer als Nebengestein. 
Daxaus geht hervor, daß es gleichgultig ist, ob das Nebengestein Sr reicher oder $5 x$ ärmer ist als die Gesteinsschliere. In jeder Falle wird bei einer vollständigen Sr-Homogenisierung awischen Schliere und Gesteinsköxper (angedeutet durch den dicken Pfeil in Abb. 8.-12) durch die Analyse der Schliere kef Beitpunkt der Homogenisierung registriert. Ein schönes Anwendungsbeispiel hat GRAUERT (1969, S. 102) durch die Analysen eines Aplitgranits aus dem Silvretta Kristallin geliefert als Beispiel für $\mathrm{Abb}$. 8. $-12 \mathrm{~A}$.

\section{$\underline{8} \cdot \underline{4} \cdot \underline{2} \cdot \underline{3}$ Die Ergebnisse_und_deren_Diskussion}

Der Aplitgang wurde entsprechend seiner chemischen Gliederung in 5 zonen (3-7) eingeteilt; an den Gesamtsteinsproben wurden $\mathrm{Rb}$ und $\mathrm{Sr}$-Isotopenanalysen durchgeführt; an einem handverlesenen, sehr reinen Blotit erfolgte eine K/Ar-Altersbestimnung. Die Ergebnisse der $\mathrm{Rb}$ und $\mathrm{Sr}$-Isotopenanalysen sind im Nicolaysen Diagramm der Abb, 8, -13 dargestellt.

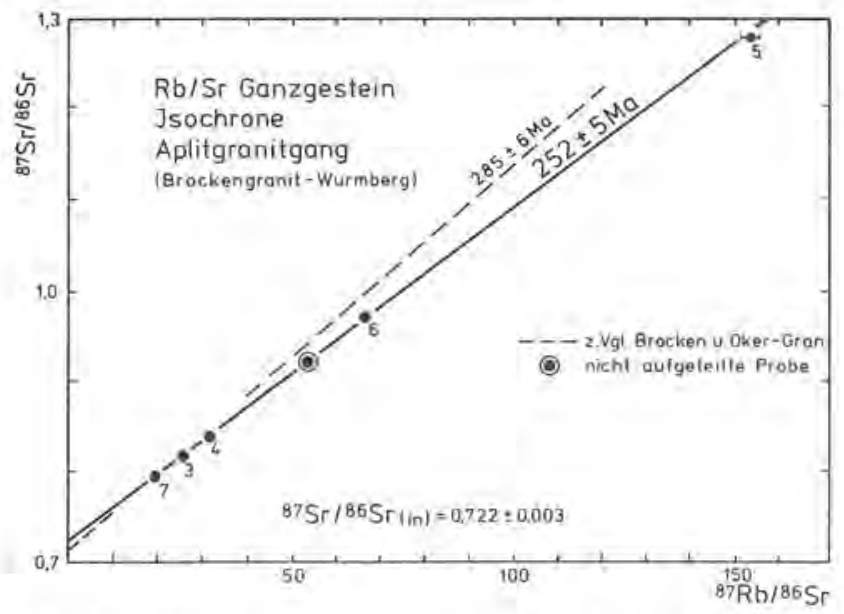

Abb. 8,-13: Die Isotopenanalysen am Aplitgranitgang im Nicolaysen Diagramm 
Anhand dieser Abbildung kann man sehr leicht folgende Yeststellungen machen, die Grundlage der folgenden Xurzen Diskussion sein müssen:

1) Meswerte der einzelnen zonen ans Apliths Im Bicolaysen Diagramm liegen auf einex Geraden.

2) Der $87 \mathrm{Sr} / 86 \mathrm{Sr}$ Anfangswart des Aplits ist böher als der des Granits $(0,722$ geqendbes 0.714$)$.

3) Die Steigung dex Isochrone tat varylichen mit dex des Granits geringer.

4) Das K/Ar-Modellalter des reinen Blotits aus dem Aplit beträgt 294 \& $5 \mathrm{ma}$ und ergab somit exakt das Modelialter des Granits.

Punkt 1) läBt sich alternativ erklären:

a) Die Gerade stelit ein reelles Modellalter dar

b) die Gerade bestimmt den Jeitpunkt eines vollkommenen Sr-Ausgleichs Granit-Aplit.

Gegen a) spricht das K/Ar Modellalter des Blotits von $294 \pm 5 \mathrm{ma}$, das im Sinne der oben genannten Definitionen als diskordant anzusprechen wäre. Weltexbin spricht der Anfangswert des Aplits von 0.722 gegen diese Möglichkeit, da wir sonst eine Differentiation saurex aplitischer Schmelzen von Granit 30-40 ma nach dessen Intrusion und Abkühlung annehmen müßten.

Somit muB die zweite Möglichkeit einex Sx-Homogenisierung angenommen werden.

Die Linearität im Nicolaysen Diagramm zejgt, daß es sich um einen vollkomenen Sx-Ausgletch Aplit-Nebengestein gehandelt haben mus. Dieser Vorgang kann sehr verständilch dargestelit werden an Hand des in Kap. 2 erwähnten Compston-Jeffery Modells, wie es in Abb. 8,-14 geschehen ist. 


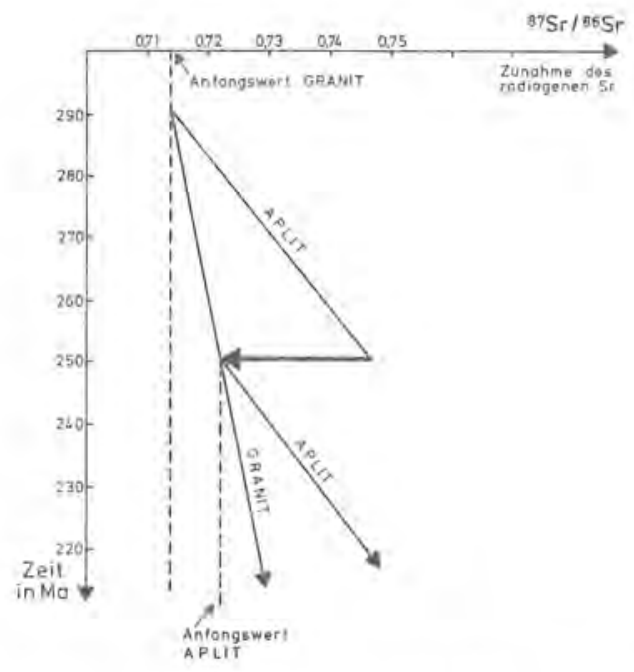

Abb. 8,-14: Darstellung der Sr-Homogenisierung des Aplitganges im Compston-Jeffery Diagramu

Dex Granit beginnt seine ${ }^{87} \mathrm{Sr} /{ }^{86}$ Sr-Entwicklung nach MaBgabe seines ${ }^{87} \mathrm{Rb}$ Gehaltes von ca. $70 \mathrm{ppm}$ bei ca, $290 \mathrm{Ma}$ una erreicht bej. $250 \mathrm{ma}$, d.h, nach 40 Ma den wert ${ }^{87} \mathrm{Sr} /{ }^{86} \mathrm{Sr}$ von 0.722 . Da der Aplitgranit einen höheren ${ }^{87} \mathrm{Rb}$-Gehalt hat, erreicht dieser in der gleichen Zeit einen ${ }^{87} \mathrm{Sr} /{ }^{86} \mathrm{Sr}$-Wert von 0.746 . In diesem Zeitpunkt findet die Sr-Homogenisierung statt und 1 äBt den ${ }^{87} \mathrm{Sr} /{ }^{86}$ Sr-wert des Aplits auf den des Granits absinken, und die Sr-Entwicklung des Aplits beginnt bei dem neuen Wert 0.722 , der als Anfangswert der Isochrone ermittelt wurde.

Diese Zusammenhänge wurden bewußt vereinfacht dargestellt, um Prinzip und Wirkung elner Sr-Homogenisierung darzustellen. Es soll auch fux weitere Untersuchungen eher als Arbeitshypothese dienen, die durch weitere Analysen bestätigt wexden muß. So 1st noch ungeklärt, welche $\mathrm{Rb} / \mathrm{Sr}$-Modellalter die Biotite haben. Weiter muß gezeigt werden, welche Mineralkomponente am Sr-Ausglelch beteiligt ist, nachdem offensichtlich das K/ArSystem dex Biotite nicht angegriffen wurde. Weitere Analysen 
müssen also noch die bisherige Modellannahme bestätigen.

\subsection{AbschlieBende geologische Interpretation}

In $\mathrm{Abb}$. 8, -15 sind die Ergebnisse der Datierungen zusammen-

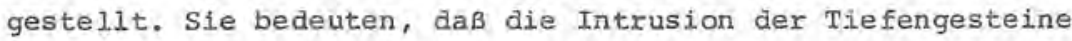
des Brocken Komplexes vor ca. 290 Ma abgeschlossen war. Die Gesteine waren zu diesem Zeitpunkt unter die kritische Diffusionstemperatur von Argon (ca. $300^{\circ} \mathrm{C}$ ) abgekühlt. Dieser Zeitpunkt läBt sich durch Vergleich mit einer stratigraphisch gesicherten K/AI Datierung aus dem Ruhrgebiet (DAMON 1968) als Grenze Westfal/Stephan bestimnen. Die Einzelmassive unterscheiden sich in ihren $\mathrm{K} / \mathrm{Ar}$ Modellaltern nicht signifikant. Da aber K/Ar Modellalter Abkuhlungsaltex sind - und das ist wichtig für jede Interpretation - kann dies nicht notwendig heiben, daß die Intrusionen gleichalt sind. Die einheitlichen $\mathrm{K} / \mathrm{Ar}$ Modellalter besagen vielmehr, daß der Bereich, in den die Brocken-Magmatite intrudierten, eine gemeinsame Abkühlungsgeschichte hatten. Die bisherigen Ergebnisse schließen nicht aus, daB z.B. der Harzbuxger Gabbro 30 Ma vor dem Brocken-oker-

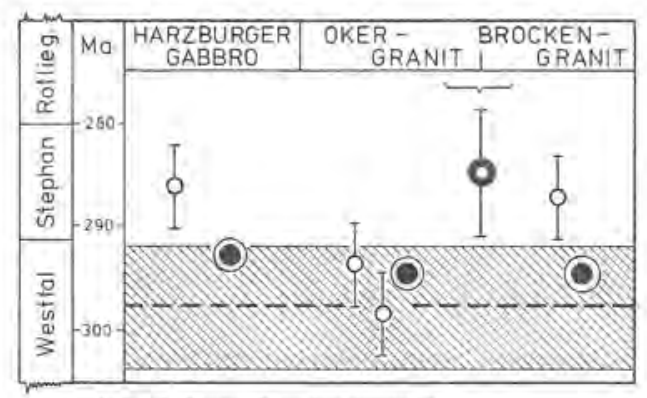

- RD,St Biotit (Einzeimadellolter)

(C) K Aar Korrigierte Madelialtor gemillolt

- Rb/5r Modellalter Gesamigesteinsisochrone lur Brocken-u. Okergranit Reterenzalter Hagen-Tonstein,
mitti Wostiol C.

Abb. 8.-15: Zusammenstellung der Modellalter 
Granit intrudierte; die Intrusion des Brocken Granits und die damit verbundene Aufheizung dieses Krustenbereiches kann die bffnung der Minerale (Argonabgabe) zur Folge gehabt haben.

In dieser Hinsicht geben die Rb/Sr-Datierungen an Biotiten keinen weiteren AufschluB uber die Intrusionsgeschichte des Harzburger Gabbros; sie bestätigen die $\mathrm{K} / \mathrm{Ar}$ Modellalter.

Auf der anderen Seite ist die Ubereinstimung innerhalb der Fehlergrenzen von $\mathrm{Rb} / \mathrm{S} r$ Ganzgesteinsdatierungen mit den $\mathrm{K} / \mathrm{Ar}$ Modellaltern von Oker- und Brocken Granit ein Hinweis dafür, daB Intrusion und Abkihlung eine Phase in der Geschichte des Granitkomplexes sind. Somit kann als gesichert angenomen werden, $d a \beta$ die eigentilche Intrusionsgeschichte des Brocken Massivs im oberen Westfal, höchstens im mittleren stephan, abgeschlossen war. - Die Gesteine und vor allem die sauren Varianten waren jedoch nach diesem Zeitpunkt 2.T. chemisch offene Systeme hinsichtlich der Elemente Rubidium und Strontium. Dies wird deutlich durch die Datierungen an einem Aplitgranit im Brocken Granit, Als ein mit den vorläufigen Daten ubereinstimmendes Arbeitsmode11 muB angenommen werden, daB im Dezimeterbereich z.T. vollkommene Sr-Homogenisierung vor $250 \mathrm{Ma}$, d.h. an der Grenze Unter Rotliegend - Ober Rotliegend, stattgefunden hat. Als geologische Ursache kann hierfür eine regionale Aufheizung der Brocken Gebiete während der Zeit des Rotliegend-Vulkanismus (Ilfelder Becken, Meisdorfer Becken) in der näheren Umgebung in Betracht kommen. 
LITERATUR

CHROBOK, S.M.: Untersuchungen zur Geologie des Brockenmassivs (Harz). - Geologie 14, Beih. 48, 82 S., 1965.

DAMON, P.E.: K/Ar dates for the Upper Carboniferons (Lower Westphalien C) Hagen Tonstein. - Annual Progress Report Nr. CO0-689-100 Contract AT (11-1)-689, S. 61-63, Tuscon, Arizon, 1968.

EVERNDEN, J.F. \& KISTLER, R.W.: Chronology of Emplacement of Mesozoic Batholithic Complexes in California and Western Nevada. - Geol. Surv. Prof. Paper 623, U.S. Government Printing Office, Washington, 1970.

FAIRBAIRN, H.W. \& HURLEY, P.M.: Northern Appalachian Geochronology as a Model for Interpreting ages in Oldex Orogens. - Eclog. Geol. Helv. 63, 1, 83-90, 1970.

FUCHS, W.: Untersuchungen $z$ ur Geologie und Petrographie des Oker-Plutons im Harz. - Clausthaler Tektonische Hefte, 9, I1I-185, 1969.

GRAUERT, B.: Die Entwicklungsgesch1chte des Silvretta-Kristallins auf Grund radiometrischer Altersbestimungen. Diss. Univ. Bern, München, 1969.

HARRE, W., KOCKEL, F., KREUZER, H., LENZ, H., MULLER, P, \& WALTHER, H,W.: Ubber Rejuvenationen im SerboMazedonischen Massiv (Deutung radiometrischer Altersbestimungen). - XXIII Internat, Geol. Congr. Vol. $\underline{6}, 223-236,1968$.

HART, S,R.: The petrology and isotopic mineral age relations of a contact zone in the Front range, Colorado. J. Geol. $72,5,493-516,1964$.

HOPPE, G., KUNERT, R. \& SCHWAB, M.: Zirkone aus Gesteinen des mitteldeutschen Permokarbons. II. Die Altersstellung des Auerbergporphyrs im Harz. - Geologie $14,7,777-813,1965$.

JÄGER, E. \& NIGGLI, E.: Rubidium-Strontium-Isotopenanalysen an Mineralien und Gesteinen des Rotondogranites und ihre geologische Interpretation. - Schweiz. Min. Petr. Mitt., 44, 61-81, 1964.

JÄGER, B.: Rb-Sr-Systems in Different Degress of Metamorphism. Eclog. Geol. Helv. 63, 1, 163-172, 1970.

MULLER, G.: Die autometamorphe retrograde Unwandlung von Biotiten in Chlorite und Muskowite in sauren Tiefengesteinen.Contr. Mineral. and Petrol. 13, 295-365, 1966. 
9. ANHANG

Die natürlicher radioaktiven zerfallsreihen (Abb, 5,-1) 
Bisher erschienen:

\section{Clausthaler Tektonische Hefte}

Heft Nr. 1, 4. Auflage, 1965; Adler, R., Fenchel, W., Hannak, W., Pilger, A., mit einem Beitrag von K.-P. Hoyer: Einige Grundlagen der Tektonik I, 64 S., 39 Abb., 2 Tab., brosch. Vergriffen, völlige Neubearbeitung des Stoffes in Heft Nr. 12

Heft Nr. 2, 3. Auflage, 1965; Adler, R., Fenchel, W., Pilger, A.: Statistische Methoden in der Tektonik I. - Die gebräuchlichsten Darstellungsarten ohne Verwendung der Lagenkugelprojektion. 97 S., 51 Abb., 9 Tab., brosch. fPr: DM 9,80

Heft Nr. 3, 3. Auflage, 1967; Adler, R., Fenchel, W., Martini, H.-J., Pilger, A.: Einige Grundlagen der Tektonik II. Die tektonischen Trennflächen. 94 S., 67 Abb., 1 Tab., brosch. fPr: DM 7,90

Heft Nr. 4, 3. Auflage, 1969; Adler, R., Fenchel, W., Pilger, A.: Statistische Methoden in der Tektonik II. Das Schmidtsche Netz und seine Anwendung im Bereich des makroskopischen Gefüges. 111 S., 79 Abb., brosch. fPr: DM 10,30

Heft Nr. 5, 1964; Karl, F.: Anwendung der Gefügekunde in der Petrotektonik, Teil I Grundbegriffe. 142 S., 73 Abb., brosch. fPr: DM 16,20

Heft Nr. 6, 1967; Kronberg, P.: Photogeologie. Eine Einführung in die geologische Luftbildauswertung. 235 S., 126 Abb., davon 46 Luftbilder und 28 Luftbildstereogramme, brosch. fPr: DM 23,-

Heft Nr. 7, 1968; Bottke, H.: Schürfbohren. Teil 1: Die Durchführung von Kernbohrungen. 140 S., 46 Abb., 60 Tab., 4 Taf., brosch. fPr: DM 21,50

Heft Nr. 8, 1968; Adler, R. E., Krückeberg, F., Pfisterer, W., Pilger, A., Schmidt, M. W.: Elektronische Datenverarbeitung in der Tektonik. 157 S., 23 Taf., 39 Abb., 24 Tab., brosch. fPr: DM 22,50

Heft Nr. 9, 1969; Fuchs, W., Günther, R., Krausse, H.-F., Meyer, W., Mohr, K., Pertold, Z., Pilger, A.: Lineamenttektonik und Magmatismus im Westharz. 260 S., 6 Tab., 32 Abb., 16 Bilder, brosch. fPr: DM 28,-

Heft Nr. 10, 1970; 28 Titel, 27 Autoren, Computer-Einsatz in der Geologie. $400 \mathrm{~S}$, 126 Abb., 13 Tab., brosch. fPr: DM 54,-

Heft Nr. 11, 1971; Geyh, Mebus, A.: Die Anwendung der ${ }^{14} \mathrm{C}$-Methode. Die Entnahme, Auswahl und Behandlung von ${ }^{14} \mathrm{C}$-Proben sowie Auswertung und Verwendung von ${ }^{14}$ C-Ergebnissen, 118 S., 12 Abb., brosch. fPr: DM 14,50

Heft Nr. 12, 1972; Flick, H., Quade, H., Stache, G.-A., mit Beiträgen von F. W. Wellmer: Einführung in die tektonischen Arbeitsmethoden. - Schichtenlagerung und bruchlose Verformung. 96 S., 54 Abb., 2 Tab., brosch. fPr: DM 10,50

Heft Nr. 13, 1972; Wendt, I., mit Beiträgen von Lenz, H. und Schoell, M.: Radiometrische Methoden in der Geochronologie. $126 \mathrm{~S}$., $47 \mathrm{Abb}$. im Text, $5 \mathrm{Tab}$. und $1 \mathrm{Abb}$. im Anhang, brosch. fPr: DM 17,50 


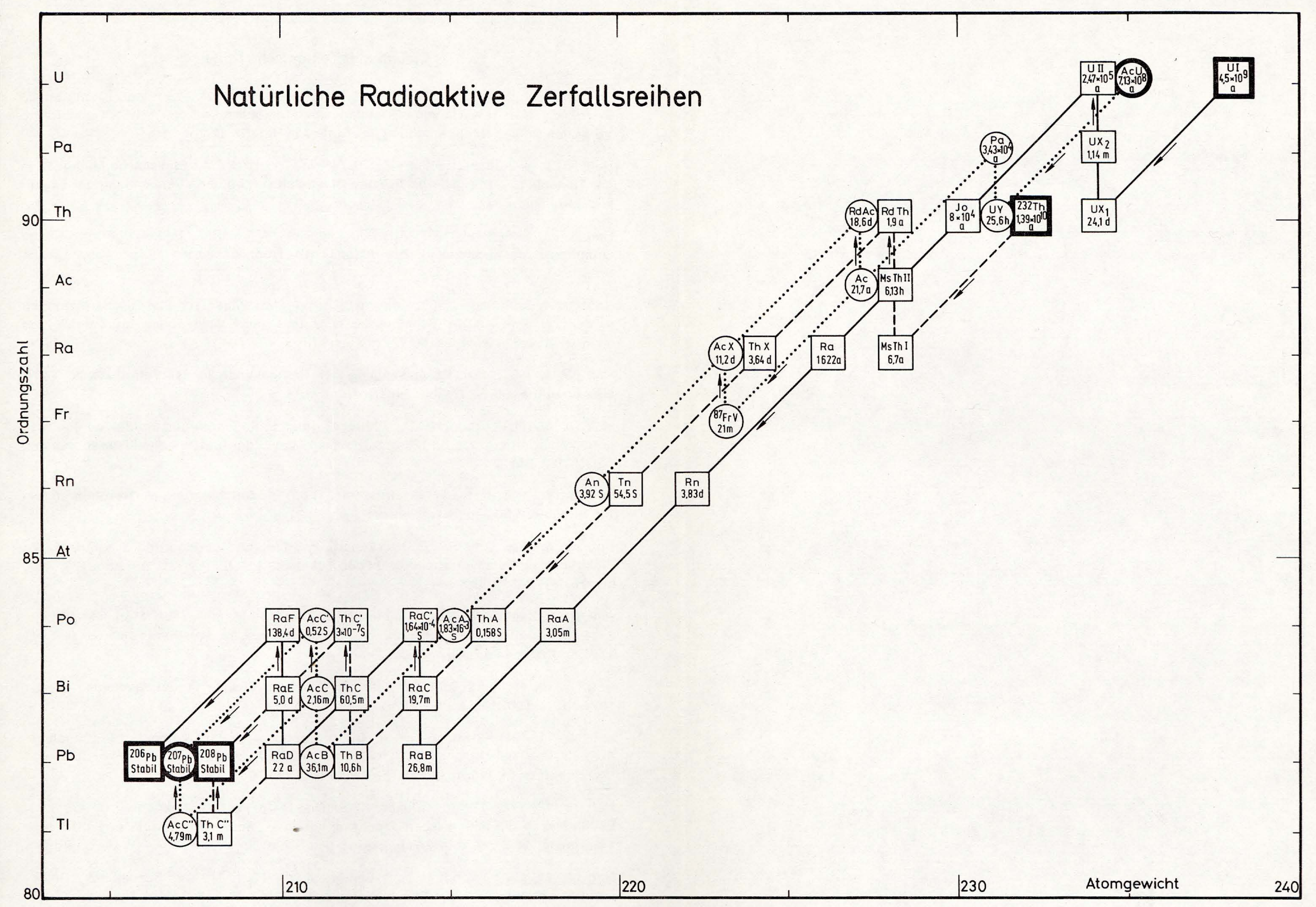


Bisher erschienen, bzw. in Vorbereitung:

\section{Clausthaler Geologische Abhandlungen}

Heft Nr. 1, 1965; Adler, R. E., Krausse, H.-F., Lautsch, H., Pilger, A.: Beiträge zur Tektonik des nördlichen Ruhrkarbons. 167 S., 62 Abb., 1 Tab., brosch. fPr: DM 10,-

Heft Nr. 2, 1965; Krausse, H.-F.: Gefügeachsen im westlichen Teil des Vestischen Hauptsattels (nördliches Ruhrgebiet) und ihre Beziehung zu den übrigen tektonischen Elementen. 114 S., 191 Abb., 7 Tab., 2 Taf., brosch. fPr: DM 10,-

Heft Nr. 3, 1970; Wellmer, F.-W.: Verwendung piezoelektrischer Messungen an quarzhaltigen Gesteinen in der Gefügekunde. 190 S., 60 Abb., 63 Tab., brosch. fPr: DM 15,-

Heft Nr. 4, 1970; Schade, H.: Der Kulm in dem nordöstlich der Lahn gelegenen Teil der Dillmulde. 178 S., 33 Abb., 2 Taf., 29 Tab., brosch. fPr: DM 15,-

Heft Nr. 5, 1970; Pilger, A., Weissenbach, N.: Stand und Aussichten der Forschung über Stratigraphie, Tektonik und Metamorphose in der Saualpe in Kärnten. 39 S., 16 Abb., brosch. fPr: DM 15,-

Heft Nr. 6, 1970; Behain, C.: Die Tektonik des Tschogart-Eisenerz-Massivs und seiner Umgebung bei Bafq im zentralen Iran. 51 S., 21 Abb., 6 Fig., 4 Tab., brosch. fPr.: DM 15,-

Heft Nr. 7, 1971; Ahmadzadeh Heravi, M.: Stratigraphische und paläontologische Untersuchungen im Unterkarbon des zentralen Elburs (Iran). 114 S., 5 Abb., 4 Taf., 4 Tab., brosch. fPr: DM 15,-

Heft Nr. 8, 1971; Pilger, A.: Die zeitlich-tektonische Entwicklung der iranischen Gebirge. 27 S., 4 Abb., 5 Taf., brosch. fPr: DM 15,--

Heft Nr. 9, 1971; Weissenbach, N.: Geologie und Petrographie der eklogitführenden hochkristallinen Serien im zentralen Teil der Saualpe, Kärnten. In Vorbereitung.

Heft Nr. 10, 1971; Podufal, P.: Zur Geologie und Lagerstättenkunde der Manganvorkommen bei Drama (Griechisch-Mazedonien). 82 S., 45 Abb., 6 Taf., brosch. fPr: DM 15,-

Heft Nr. 11, 1971; Klarr, K.: Der geologische Bau des südöstlichen Teiles vom AldudesQuinto Real-Massiv (spanische Westpyrenäen). 148 S., 42 Abb., 1 Tab., 16 Beil., brosch. fPr: DM 15,-, z. Z. vergriffen

Heft Nr. 12, Magnesitvorkommen in den Westpyrenäen (vorl. Titel), in Vorbereitung

Heft Nr. 13, 1972; Requadt, H.: Zur Stratigraphie und Fazies des Unter- und Mittel. devons in den spanischen Westpyrenäen. $113 \mathrm{~S}$., 40 Abb., 1 Taf., brosch. fPr: DM 10,-

Heft Nr. 14, 1972; Dennert, H., Hintze, J., Mohr, K. und Pilger, A.: Gangkarte des Stadtgebietes von Clausthal-Zellerfeld, 1:10000, mit Erläuterungen, 38 S., 7 Abb., 2 Taf., brosch. fPr: DM 10,- 
Bisher erschienen, bzw. in Vorbereitung:

\section{Clausthaler Geologische Abhandlungen}

Heft Nr. 1, 1965; Adler, R. E., Krausse, H.-F., Lautsch, H., Pilger, A.: Beiträge zur Tektonik des nördlichen Ruhrkarbons. 167 S., 62 Abb., 1 Tab., brosch. IPr; DM 10,-

Heft Nr. 2, 1965; Krausse, H.-F.; Gefügeachsen im westlichen Teil des Vestischen Hauptsattels (nördliches Ruhrgebiet) und ihre Beziehung zu den übrigen tektonischen Elementen. 114 S., 191 Abb., 7 Tab., 2 Tal., brosch. fPr: DM 10,-

Heft Nr. 3, 1970: Wellmer, F.-W.: Verwendung piezoelektrischer Messungen an quarzhaltigen Gesteinen in der Gefügekunde. 190 S., 60 Abb., 63 Tab., brosch. PPr: DM 15,-

Heft Nr. 4, 1970; Schade, H.; Der Kulm in dem nordöstlich der Lahn gelegenen Teil der Dillmulde. 178 S., 33 Abb., 2 Taf., 29 Tab., brosch. Pr: DM 15,-

Heft Nr. 5, 1970; Pilger, A., Weissenbach, N.: Stand und Aussichten der Forschung über Stratigraphie, Tektonik und Metamorphose in der Saualpe in Kärnten. 39 S., 16 Abb., brosch. FPr: DM 15,-

Heft Nr. 6, 1970; Behain, C.: Die Tektonik des Tschogart-Eisenerz-Massivs und seiner Umgebung bei Bafq im zentralen Iran. 51 S., 21 Abb., 6 Fig. 4 Tab., brosch. fPr.: DM 15,-

Heft Nr. 7, 1971; Ahmadzadeh Heravi, M.: Stratigraphische und paläontologische Untersuchungen im Unterkarbon des zentralen Elburs (Iran). 114 S., 5 Abb., 4 Taf., 4 Tab.. brosch. fPr: DM 15,-

Heft Nr. 8, 1971; Pilger, A.: Die zeitlich-tektonische Entwicklung der iranischen Gebirge. $27 \mathrm{~S}$., $4 \mathrm{Abb}, 5$ Taf., brosch. fPr: DM 15,-

Heft Nr. 9, 1971: Weissenbach, N.: Geologie und Petrographie der eklogitführenden hochkrisłallinen Serien im zentralen Teil der Sauaipe, Kärnten. In Vorbereitung.

Heft Nr. 10, 1971; Podufal,P.: Zur Geologie und Lagerstättenkunde der Manganvorkommen bei Drama (Griechisch-Mazedonien). 82 S.: 45 Abb, 6 Taf., brosch. PPr: DM 15,-

Heft Nr. 11, 1971; Klarr, K.: Der geologische Bau des südöstlichen Teiles vom AldudesQuinto Real-Massiv (spanische Westpyrenäen). 148 S., 42 Abb., 1 Tab., 16 Beil., brosch. IPr: DM $15,-$, z. Z. vergriffen

Heft $\mathrm{Nr}$. 12, Magnesitvorkommen in den Westpyrenäen (vorl. Titel), in Vorbereitung

Heft Nr. 13, 1972; Requadt. H.: Zur Stratigraphie und Fazies des Unter- und Mittel. devons in den spanischen Westpyrenäen. 113 S., 40 Abb., 1 Taf., brosch. fPr: DM 10,-

Heft Nr. 14, 1972; Dennert, H., Hintze, J., Mohr. K. und Pilger, A.: Gangkarte des Stadtgebietes von Clausthal-Zellerfeid, 1:10000. mit Erläuterungen, 38 S., 7 Abb., 2 Taf., brosch. fPr: DM 10,- 


\title{
Series on Rock and Soil Mechanics
}

\author{
Vol. $1(1971 / 73)$ No. 2 \& 3
}

W. Dreyer, TU Clausthal:

THE SCIENCE OF ROCK MECHANICS

Part I Strength Properties of Rocks

1972, 500 Seiten, 137 Abb., 86 Tafeln, Preis: US Dollar 25.00 geb.

"Das Buch ist nicht nur für die Ingenieurgeologie von Interesse, sondern kann jedem Bau- und Bergingenieur, der sich im Rahmen von Felsarbeiten mit den Festigkeitseigenschaften von Gestein und Gebirge befassen muß, empfohlen werden.«

Geologische Mitteilungen, Juli 1972

»Es ist zu erwarten, daß das vorliegende Buch bei Gebirgsmechanikern international große Bedeutung finden wird.«

Kali und Steinsalz, Februar 1972

"Mit diesem Buch finden diese gründlichen Untersuchungen im Rahmen einer deutschen Hochschule nunmehr Eingang in das internationale Schrifttum. Allein schon für diese Tatsache sollte man dem Verlag und dem wohlrenommierten Verfasser dankbar sein.«

Erdöl und Kohle, August 1972

T. H. Hanna, University of Sheffield:

FOUNDATION INSTRUMENTATION

Januar 1973, 350 Seiten, 251 Abb., Preis: US Dollar 30.00 geb.

This book shows how the foundation engineer may benefit from the use of field instrumentation. This topic has become increasingly important over the past few years and a need for a comprehensive text on the principles of operation, installation and use of the various instruments and data processing systems has arisen. This text will help to establish field instrumentation as a respectable and necessary part of foundation design, construction and performance evaluation.

Bestellen Sie diese wichtigen Bücher heute oder fordern ausführliche Prospekte an:

TRANS TECH S. A.

$\mathrm{CH}-4711$ Aedermannsdorf (Schweiz) 